Portland State University

PDXScholar

$1-1-2011$

\title{
Model-Based Information Extraction From Synthetic Aperture Radar Signals
}

Shari Matzner

Portland State University

Follow this and additional works at: https://pdxscholar.library.pdx.edu/open_access_etds Let us know how access to this document benefits you.

\section{Recommended Citation}

Matzner, Shari, "Model-Based Information Extraction From Synthetic Aperture Radar Signals" (2011). Dissertations and Theses. Paper 248.

https://doi.org/10.15760/etd.248

This Dissertation is brought to you for free and open access. It has been accepted for inclusion in Dissertations and Theses by an authorized administrator of PDXScholar. Please contact us if we can make this document more accessible: pdxscholar@pdx.edu. 
Model-Based Information Extraction

From Synthetic Aperture Radar Signals

by

Shari A. Matzner

A dissertation submitted in partial fulfillment of the requirements for the degree of

\author{
Doctor of Philosophy \\ in \\ Systems Science
}

\author{
Dissertation Committee: \\ Lisa M. Zurk, Chair \\ George G. Lendaris \\ James McNames \\ Daniel Rouseff \\ Gerardo Lafferriere
}

Portland State University

(c) 2011 


\begin{abstract}
Synthetic aperture radar (SAR) is a remote sensing technology for imaging areas of the earth's surface. SAR has been successfully used for monitoring characteristics of the natural environment such as land cover type and tree density. With the advent of higher resolution sensors, it is now theoretically possible to extract information about individual structures such as buildings from SAR imagery. This information could be used for disaster response and security-related intelligence. SAR has an advantage over other remote sensing technologies for these applications because SAR data can be collected during the night and in rainy or cloudy conditions.
\end{abstract}

This research presents a model-based method for extracting information about a building - its height and roof slope - from a single SAR image. Other methods require multiple images or ancillary data from specialized sensors, making them less practical. The model-based method uses simulation to match a hypothesized building to an observed SAR image. The degree to which a simulation matches the observed data is measured by mutual information. The success of this method depends on the accuracy of the simulation and on the reliability of the mutual information similarity measure.

Electromagnetic theory was applied to relate a building's physical characteristics to the features present in a SAR image. This understanding was used to quantify the precision of building information contained in SAR data, and to identify the inputs needed for accurate simulation. A new SAR simulation technique was developed to meet the accuracy and efficiency requirements of model-based information extraction.

Mutual information, a concept from information theory, has become a standard for 
measuring the similarity between medical images. Its performance in the context of matching a simulation image to a SAR image was evaluated in this research, and it was found to perform well under certain conditions. The factors that affect its performance, and the model-based method overall, were found to include the size of the building and its orientation. Further refinements that expand the range of operational conditions for the method would lead to a practical tool for collecting information about buildings using SAR technology.

This research was performed using SAR data from MIT-Lincoln Laboratory. 


\section{DEDICATION}

This dissertation is dedicated to my father, Peter Matzner. 


\section{ACKNOWLEDGMENTS}

I would like to thank MIT-Lincoln Laboratory for supplying funding and data, without which this work would not have been possible. I would also like to thank the Pacific Northwest National Laboratory for providing financial support and computing resources, and my colleagues there for providing invaluable encouragement. Thanks are also due to my former fellow students at the NEAR-Lab for their technical assistance and general camaraderie - in particular, Reto Toengi and George Ogden. And my warmest gratitude to Tad Shannon, whose friendship and collaboration I will always remember.

The members of my committee deserve special thanks for seeing me through the process of completing this dissertation. Daniel Rouseff, who made an extra commitment to participate in this process, was always responsive with helpful advice and kind words. James McNames introduced me to statistical signal processing and set me on the path I'm following today. From the beginning of my studies to the end, George Lendaris - to me, the embodiment of Systems Science - has been a source of intellectual inspiration. My adviser, Lisa Zurk, has contributed immeasurably to my development on many levels; her influence will continue to guide me in my professional pursuits and I am honored to consider her a colleague and a friend. I cannot thank her enough.

Finally, my loving gratitude to my partner, David Brande, for his editing help, his patience and his unflagging support. 


\section{Contents}

Abstract $\quad$ i

Dedication $\quad$ iii

Acknowledgments $\quad$ iv

List of Tables viii

List of Figures $\quad$ ix

List of Symbols $\quad$ xii

1 Introduction 1

1.1 Research Problem . . . . . . . . . . . . . . . . . . . . 1

1.2 Systems Approach . . . . . . . . . . . . . . . . . . 3

1.3 Contributions of This Research . . . . . . . . . . . . . 5

2 Literature Review $\quad 7$

2.1 Extraction of Building Information from SAR Data . . . . . . . 7

2.2 Electromagnetic Models for Buildings . . . . . . . . . . . . . . . . . 10 
2.3 SAR Simulation . . . . . . . . . . . . . . . . . 11

2.4 Mutual Information as a Similarity Measure . . . . . . . . . . . 13

3 Theoretical Background $\quad 16$

3.1 Electromagnetic Theory .................. 16

3.2 Spotlight Synthetic Aperture Radar . . . . . . . . . . . . . . 23

3.2.1 The LiMIT SAR Sensor . . . . . . . . . . . . . . . 34

3.3 Information Theory . . . . . . . . . . . . . . . 38

4 Building Features in SAR Imagery $\quad 41$

4.1 Characterization of Building Scattering . . . . . . . . . . . 42

4.1.1 Intensity Of Scattering From Building Components . . . . . . 43

4.1.2 Scattering Components Mapped To Image Features . . . . . . 49

4.2 Interpretation of Buildings in LiMIT Data . . . . . . . . . . . 58

4.2.1 Buildings in LiMIT Data . . . . . . . . . . . . . . 58

4.2.2 Analysis of Building Image Features . . . . . . . . . . . . . . . 61

4.2.3 Model-based Interpretation of Features . . . . . . . . . . . . 66

4.3 Summary . . . . . . . . . . . . . . . . . . 69

5 Simulation of SAR Signals $\quad 70$

5.1 New SAR Simulation Method . . . . . . . . . . . . . 70

5.1.1 Phase History Evaluation . . . . . . . . . . . . 73

5.1 .2 Raw Signal Reconstruction . . . . . . . . . . . . . 75 
5.1 .3 Computational Cost . . . . . . . . . . . . 78

5.2 Simulation Results . . . . . . . . . . . . . . . . 80

5.2 .1 Simulation of Buildings . . . . . . . . . . . . . . . 81

5.2 .2 Evaluation of Building Feature Model . . . . . . . . . . . 84

5.3 Summary . . . . . . . . . . . . . . . . . . . . . 89

6 Mutual Information Method For Information Extraction 90

6.1 Theoretical Considerations . . . . . . . . . . . . . . . . 91

6.1.1 Probability Distribution Estimation . . . . . . . . . . . 92

6.1.2 Mutual Information Normalization _. . . . . . . . . . 95

6.2 Application to LiMIT Data . . . . . . . . . . . . . 96

6.2 .1 Methodology . . . . . . . . . . . . . . . . 97

6.2 .2 Case 1: L-Building . . . . . . . . . . . . . . . 100

6.2 .3 Case 2: Barns . . . . . . . . . . . . . . . . . . 102

6.3 Summary . . . . . . . . . . . . . . . . . . . 107

$\begin{array}{lll}7 \text { Conclusion } & 109\end{array}$

7.1 Summary of Contributions . . . . . . . . . . . . . . . 109

7.2 Future Work . . . . . . . . . . . . . . . . . . . . 111

$\begin{array}{ll}\text { Bibliography } & 113\end{array}$ 


\section{List of Tables}

3.1 LiMIT Parameters . . . . . . . . . . . . . . . . . 36

4.1 Common Roof Slopes in the United States . . . . . . . . . . . . 43

4.2 Dielectric Constants and Roughness . . . . . . . . . . . . . . 47

4.3 Building Points in Ground Coordinates . . . . . . . . . . . . . . . . . 52

4.4 LiMIT CPI Geometry . . . . . . . . . . . . . . . . . 59

4.5 L-Building Parameters . . . . . . . . . . . . . . . . . 60

4.6 Barn Parameters . . . . . . . . . . . . . . . . . . 60

4.7 Summary of range profile analysis. . . . . . . . . . . . 65

5.1 Sensor Parameters For Simulation . . . . . . . . . . . . . . . . 74

5.2 Computational Cost Factors . . . . . . . . . . . . . . . . . 78

5.3 Simulation Wall Feature . . . . . . . . . . . . . . 87

5.4 Simulation Roof Feature . . . . . . . . . . . . . . . . . 88

6.1 Barn Height Extraction Results . . . . . . . . . . . . . . . . 106 


\section{List of Figures}

3.1 Electromagnetic wave. . . . . . . . . . . . . . . . 17

3.2 Electromagnetic scattering. . . . . . . . . . . . . . . . 18

3.3 The scattering from a dihedral. . . . . . . . . . . . . . 23

3.4 SAR spotlight mode data collection. . . . . . . . . . . 25

3.5 Phase history domain. . . . . . . . . . . . . . . . . . . . . . . . . . . 29

3.6 Echoes from a single transmitted pulse. . . . . . . . . . . . . 32

3.7 LiMIT sensor. . . . . . . . . . . . . . . . . . . . . . . . . . . . . 34

3.8 LiMIT reference signal. . . . . . . . . . . . . . . . . . . . . . . 35

3.9 Example LiMIT images. . . . . . . . . . . . . . . . . . . . . 37

3.10 Mutual information. . . . . . . . . . . . . . . . 40

4.1 Building model symbol definition. . . . . . . . . . . . . . 42

4.2 Scattering intensity vs incident angle. . . . . . . . . . . 45

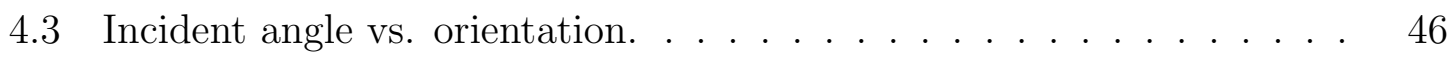

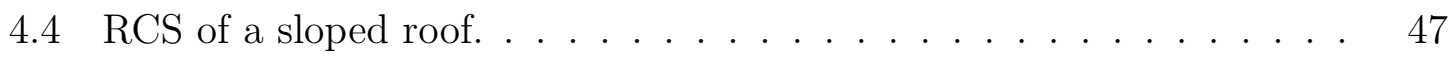

4.5 RCS of a vertical wall and standard sloped roof. . . . . . . . . 48

4.6 RCS of double bounce. . . . . . . . . . . . . . . . . . . 49 
4.7 Relative RCS of building components. . . . . . . . . . . . . . . . . 50

4.8 Building signature influenced by shape and grazing angle. . . . . . . 50

4.9 Building points for geometric model. . . . . . . . . . . . . . . . 52

4.10 Difference between aspect angle in scene and in image. . . . . . . . . 54

4.11 Building parameter resolution. . . . . . . . . . . . . . 57

4.12 Buildings in LiMIT imagery. . . . . . . . . . . . . . . . . . . 59

4.13 Barn parameters. . . . . . . . . . . . . . . . 61

4.14 Range profile locations. . . . . . . . . . . . . . . . . . . . . 62

4.15 Range profile analysis of L-building. . . . . . . . . . . . . . 63

4.16 Range profile analysis of barns. . . . . . . . . . . . . . . . 64

4.17 Possible L-building shapes. . . . . . . . . . . . . . . . . . . . . 68

5.1 Phase-domain simulation method. . . . . . . . . . . . . . . . . 72

5.2 Upsampled signal. . . . . . . . . . . . . . . . . . . . 76

5.3 Test scene verification of simulation. . . . . . . . . . . . . 77

5.4 Simulated building layout. . . . . . . . . . . . . . . . . 81

5.5 Simulated building, side-view. . . . . . . . . . . . . . 82

5.6 Example synthetic building scenes. . . . . . . . . . . . . . 83

5.7 Barn simulation. . . . . . . . . . . . . . . . . . 83

5.8 L-Building simulation. . . . . . . . . . . . . . . . . . . . 84

5.9 Simulation of possible L-building shapes. . . . . . . . . . . . . . . 85

5.10 Simulated vertical wall image. . . . . . . . . . . . . . . . 86 
5.11 Simulated roof image. . . . . . . . . . . . . . . . . . 88

6.1 Effect of bin width. . . . . . . . . . . . . . . . . . 94

6.2 Binned images. . . . . . . . . . . . . . . . . . 95

6.3 LiMIT SAR images of L-building. . . . . . . . . . . . . . . . 101

6.4 Registration of simulation image with LiMIT image. . . . . . . . . . . 101

6.5 Similarity maps for L-building. . . . . . . . . . . . . . . . 103

6.6 LiMIT SAR images of barns. . . . . . . . . . . . . . . . . 104

6.7 Registration examples for barns. . . . . . . . . . . . . . . . . 105

6.8 Barn height estimate histograms. . . . . . . . . . . . . . 105

6.9 Similarity maps for barns. . . . . . . . . . . . . . . . . . . 106 


\section{List of Symbols}

\section{Constants}

\begin{tabular}{ll} 
Symbol & Description \\
\hline$c$ & speed of light $(\mathrm{m} / \mathrm{s})$ \\
$\mathrm{e}$ & base of natural logarithm (unitless) \\
$\mathrm{j}$ & square root of -1
\end{tabular}

\section{Variables}

\begin{tabular}{ll} 
Symbol & Description \\
\hline$D_{x}, D_{y}$ & scene size in cross-range and range $(\mathrm{m})$ \\
$\mathbf{E}_{i}$ & incident electromagnetic field \\
$\mathbf{E}_{s}$ & scattered electromagnetic field \\
$H$ & building height $(\mathrm{m})$ \\
$\tilde{H}$ & image wall feature width in the range dimension $(\mathrm{m})$ \\
$L$ & building length in the cross-range dimension $(\mathrm{m})$ \\
$\overrightarrow{\boldsymbol{R}}$ & SAR sensor position \\
$T$ & SAR transmitted pulse duration $(\mathrm{sec})$ \\
$W$ & building width in the range dimension $(\mathrm{m})$ \\
$\tilde{W}$ & image roof feature width in the range dimension $(\mathrm{m})$ \\
$\boldsymbol{b}$ & building parameter vector \\
$f$ & frequency (Hz) \\
$k$ & spatial frequency, wavenumber (rad $/ \mathrm{m})$ \\
$\hat{\boldsymbol{k}}$ & wave propagation direction (unit vector) \\
$\hat{\boldsymbol{n}}$ & surface normal (unit vector) \\
$r$ & distance (m) \\
$\overrightarrow{\boldsymbol{r}}$ & scatterer position \\
$t$ & time (s) \\
$\mathrm{v}$ & a two-dimensional image, a random variable \\
$\mathrm{v}_{0}$ & SAR image \\
$\mathbf{v}_{\boldsymbol{b}}$ & simulation image \\
$x, y, z$ & scene coordinates (m) \\
$\tilde{x}, \tilde{y}$ & image coordinates (m) \\
$\alpha$ & 1/2 the chirp rate of the transmitted pulse $\left(\mathrm{rad} / \mathrm{sec}^{2}\right)$ \\
$\beta$ & bandwidth (Hz) \\
$\gamma$ & reflectivity, radar cross section \\
$\epsilon_{r}$ & image pixel intensity \\
& relative permittivity (complex value) \\
&
\end{tabular}


$\theta \quad$ SAR pulse view angle (rad)

$\lambda \quad$ wavelength $(\mathrm{m})$

$\rho_{x}, \rho_{y} \quad$ cross-range and range resolution of SAR data (m)

$\phi \quad$ building orientation (rad)

$\varphi \quad$ SAR tilt angle (rad)

$\psi \quad$ SAR grazing angle (rad)

$\omega \quad$ angular frequency $(\mathrm{rad} / \mathrm{sec})$

\section{Functions}

Notation Description

$F(k, \theta) \quad$ SAR phase history

$\mathcal{H}(a) \quad$ Shannon entropy of a random variable

$\mathcal{H}(a \mid b) \quad$ conditional entropy

$\mathcal{H}(a, b) \quad$ joint entropy of two random variables

$\mathcal{I}(a, b) \quad$ mutual information between two random variables

$p(t) \quad$ SAR transmitted pulse

$p_{0}(t) \quad$ SAR reference signal

$\mathcal{P}(a) \quad$ probability density function of a random variable

$\mathcal{P}(a \mid b) \quad$ conditional probability density function

$\mathcal{P}(a, b) \quad$ joint probability density function of two random variables

$s_{m}(t) \quad$ SAR raw signal from the $m$ th pulse 


\section{Chapter 1}

\section{Introduction}

\subsection{Research Problem}

Synthetic Aperture Radar (SAR) is a remote sensing technology for imaging areas of the earth's surface. The SAR image is synthesized by coherently combining multiple radar measurements acquired along a sensor trajectory; this produces an image with resolution akin to that produced by an array of sensors. The resulting two-dimensional image is a map of the surface's electromagnetic reflectivity.

Historically, SAR has been used to monitor the natural environment, where characteristics such as land cover, vegetation density and soil moisture content can be inferred from the intensity and texture statistics of SAR imagery. There are now SAR sensors capable of producing high resolution imagery, on the order of a meter or better, that could be used, theoretically, for extracting information about individual structures such as buildings. Information that could be obtained from high-resolution SAR images includes knowledge of whether or not buildings are present in a scene, 
what types of buildings are present, and whether a building has changed in some way.

Extracting this sort of information from SAR data is motivated by a variety of sensing needs, from intelligence and surveillance operations to the need to respond to natural disasters that impact population centers. SAR has an advantage over optical and infrared sensors for these applications because it can operate day or night and in cloudy conditions. However, a lack of understanding of the electromagnetic signatures of anthropogenic structures has prevented the full exploitation of SAR technology for these purposes.

Despite the higher resolution, extracting information about a specific structure, such as a building, from SAR imagery remains a difficult problem. Feature detection algorithms for optical imagery are not effective for SAR imagery because the two sensing modalities produce different responses. The electromagnetic response of structures commonly found in buildings is highly dependent on the orientation of the building relative to the sensor. The projection of a three-dimensional building into a twodimensional SAR image produces effects such as layover, shearing and foreshortening that distort the appearance of the building in the image. Models are needed to relate a building's physical attributes to its electromagnetic response and to identify the information available in the image features produced by a building. The questions addressed in this research are 1) how do the attributes of a building affect its appearance in SAR imagery? and 2) how can this understanding be used to extract information? 


\section{$1.2 \quad$ Systems Approach}

The approach taken in this research is a model-based approach that is informed by the practices and concepts of systems science. A definition of the system [1] of elements and relations that produces a SAR image of a building provides a context for interpreting the features observed in the image. At the highest level of abstraction, the system is composed of two subsystems, the SAR sensor and a building; these subsystems exist in an environment composed of the ground surrounding the building and the volume of space containing the sensor's trajectory and the building scene. Models of the subsystem elements and their interactions were used to capture the available a priori knowledge relevant to the problem, and to understand the relationship between a building and its features in a SAR image.

The first component of this research is the characterization of the electromagnetic response of a building in the context of the SAR imaging process. Using the a priori knowledge provided by electromagnetic principles, the attributes of the building and ground that influence the response were investigated. Effects specific to SAR signal processing were incorporated to further characterize the SAR signature of a building. The characterization identified a minimal level of abstraction for modeling the building and the surrounding ground that was sufficient to explain SAR image features. The resulting understanding was applied to the interpretation of features observed in SAR imagery.

The second component of this research is the simulation of SAR signals. In systems science, simulation is used to validate assumptions and to generate experimental results for inductive analysis. Here, a simulator was constructed to implement

the system composed of the SAR sensor and the building scene. The building and 
surrounding ground were modeled based on the results of the electromagnetic characterization. The SAR sensor was modeled based on a priori knowledge of the SAR measurement process. Simulation was used to validate the building response characterization by reproducing observed SAR signatures of buildings. Simulation was also used to analyze the change in image features produced by changes in building parameters.

The third component of this research is extracting information. This was accomplished by comparing a SAR image to simulation images using mutual information as the similarity measure. Information is defined as a reduction in uncertainty; here, uncertainty about the size and shape of a building is reduced by generating a similarity map in building parameter space. The formulation of this approach was guided by machine learning practices: specifically, considering the space of all possible building shapes, then using a priori knowledge to constrain the space and choosing an appropriate measure for characterizing the space in the context of the problem. The mutual information similarity measure was taken from information theory, a component of systems science.

The data used for this research comes from the MIT Lincoln Laboratory Multi-mission ISR $^{1}$ Testbed (LiMIT). The LiMIT SAR sensor data were available in raw signal form, along with the sensor navigation data from each collection. This dataset was used to validate the building signature model, to verify simulation results and to evaluate the proposed information extraction method.

\footnotetext{
${ }^{1}$ Intelligence, Surveilance and Reconnaisance
} 


\subsection{Contributions of This Research}

- Developed new models relating a building's physical parameters to its SAR image features. The models, in the form of equations, incorporate the geometric transformation from three dimensions to two dimensions that is inherent in the SAR imaging process. These models contribute to information extraction in two ways. First, they provide a mechanism for calculating a building's height, width and roof slope from observed image features. Second, they quantify the precision of building parameter information available in SAR data.

- Identified attributes of a building that influence its electromagnetic response. The electromagnetic response of a building is typically decomposed into the reflections from structural components such as the walls and the roof. Using electromagnetic principles, the intensities of these component reflections were compared under different conditions. The results show that the relative intensities are influenced not only by the orientation of the building relative to the sensor, but also by the slope of the building's roof and the ground cover surrounding the building. This understanding aids SAR image interpretation, where observed image features are attributed to structural components of a building.

- Demonstrated a mutual information method for extracting building information from SAR imagery. The demonstration shows that mutual information and simulation can be used to extract information about a building's height and the slope of its roof from a single SAR intensity image. This is an advance of previous work that extracted only height information, or that estimated roof slope from multiple images. 
- Developed a new technique for simulating SAR signals. Simulation is a key part of the information extraction method used here. For this purpose, the simulation must accurately reproduce the signal measurements from a particular SAR data collection. The simulation must also be efficient so that multiple simulations can be generated to find the best match between an hypothesized building shape and an observed SAR building image. The new simulation method developed here satisfies both these criteria, unlike other methods which achieve efficiency at the expense of accuracy. 


\section{Chapter 2}

\section{Literature Review}

This research addresses the problem of extracting information about buildings from SAR data. The approach is to model the SAR signature of a building and to apply the model by matching a parameterized simulation to a SAR image using mutual information as the similarity measure. The literature review is divided into sections addressing the main aspects of this problem and approach.

\subsection{Extraction of Building Information from SAR Data}

Approaches to extracting information about buildings from SAR data vary with the type of information that is desired. Building reconstruction is the term used to describe estimating a building's size and shape, and this is the problem considered here. The approaches to reconstruction vary in the number of images that are used, the use of complementary data from other sources, and how hypotheses are generated and selected. The approaches can be grouped into shadow-based methods, featurebased methods and model-based methods. 
Shadow-based methods have been used for building reconstruction from optical images $[2,3,4]$ and also from SAR imagery $[5,6,7,8,9]$. These methods use edge or contour detection to delineate the shadows and then reconstruct the building shape using the geometry of the imaging process. The most difficult challenge is to accurately delineate the shadow boundaries, which are distorted by speckle noise and which may be occluded by other structures and vegetation.

The feature-based method of reconstruction consists of identifying candidate features in an image and then combining them to form corners and parallelograms, filling in gaps and missing edges according to rules about the expected shape of a building $[10,11,12]$. The success of this approach depends on the correct delineation of features, and suffers from the same difficulties as shadow-based methods.

Both shadow-based and feature-based methods work best with images from multiple aspect angles $[6,8,12]$ so that complementary features are present in each image. Processing multiple images requires more computation than processing a single image, and acquiring multi-aspect data sets requires more resources. These two methods also rely on image processing techniques such as line detection $[11,13]$, perceptual grouping [14], and morphological operations [5]; the underlying electromagnetic principles of SAR are not exploited.

Model-based reconstruction, a more recent approach, explicitly incorporates knowledge of the SAR imaging process and electromagnetic theory. The model-based reconstruction approach is rooted in systems science. Luttrell presents the perspective that data only has meaning in the context of some model encoding prior knowledge; this perspective is then applied to the interpretation of SAR data [15]. Luttrell's view is the constructivist view underlying much of systems science [16]. The use 
of models for data interpretation was also advocated by Franceschetti $[17,18]$, who made important contributions to modeling the electromagnetic response of buildings and who demonstrated the usefulness of simulation for interpreting SAR imagery of urban areas. This work will be discussed in more detail in later sections. Modelbased reconstruction of buildings is still in the early stages of development. This is the approach taken here, based on promising reported results.

One example is demonstrated by Thiele, who related the SAR signature of a building to the building's height, width and roof slope $[19,20]$. The relationship results in an ambiguity between two hypotheses for the building shape that could both produce the same signature. The ambiguity was resolved by simulating the interferometric phase $^{1}$ for each hypothesized shape and comparing it to the observed interferometric phase. The reported building height estimates were on average $25 \%$ greater than the true heights so there remains room for improvement. The modeled relationship between the SAR signature and the building shape assumed that the building was oriented broadside to the sensor and did not account for differences in the intensity of electromagnetic responses from different parts of the building. Thiele's method also has the disadvantage of requiring interferometric phase data.

Brunner proposed a model-based method that requires only a single SAR image $[21,22]$. The SAR image of a building is matched to a simulated image of a building, and the parameters of the building are estimated as those of the simulation that best matches the actual image. The matching function was the mutual information between the simulated image and the SAR image. The simulation employed by Brunner did use electromagnetic principles, but captured the geometric effects of the SAR

\footnotetext{
${ }^{1}$ Interferometric SAR uses two receivers and the phase difference between the received signals provides surface height information.
} 
imaging process. Buildings were a priori identified as having a flat roof or sloped roof and only the height was varied during the matching process. The reported error of the height estimate on a set of 38 buildings was normally distributed with an interquartile range of -2 to +2 meters. It is difficult to judge these results without a theoretical bound on achievable precision.

In this research, the work of Thiele was extended to formulate a more general model relating the features of a building's SAR signature to the building's height, width and roof slope for any building orientation. The work of Brunner was extended to incorporate a more physically accurate simulation based on the models of Franceschetti and to match both height and roof slope, thereby reducing the amount of required a priori information. Also, the precision with which building parameters can be estimated from SAR data was quantified.

\subsection{Electromagnetic Models for Buildings}

The SAR imaging process measures a building's electromagnetic response. Modeling this response is important for understanding the information about a building that is available in SAR data. It has been shown both theoretically and empirically that the dominant components of a building's electromagnetic response are the reflections from the roof, the vertical walls, and the dihedral formed by a wall and the ground $[23,24]$. The relative intensities of these components depend on electromagneticrelated factors that are summarized by Xia [24]. These factors include the radar wavelength, building orientation relative to the radar, and the material properties of both the building and the surrounding terrain. 
In the classic paper by Franceschetti [25], models were developed for the electromagnetic response of a vertical wall and of a dihedral wall-ground structure. These models have been found to agree with empirical measurements [26, 23, 27, 28], and have been demonstrated to be useful for extracting information from SAR data [29, 30, 31].

Despite the proven validity of Franceschetti's models, an alternative model is sometimes used for SAR simulation [32, 21, 33]. This model is referred to as Lambert's Law or the Lambertian model and it comes from the field of optics. The advantage of this model is its computational simplicity but the validity of this model for electromagnetic responses in the context of SAR imaging has not been established.

In this research, the models of Franceschetti were used to investigate the effects of building orientation on the relative intensities of the building's electromagnetic response. The work of Franceschetti was extended here to consider the reflection from a sloped roof in addition to the reflections from the walls and wall-ground dihedral. The Lambertian model was found to produce results that differed significantly from those of Franceschetti's models.

\subsection{SAR Simulation}

Simulation plays a crucial role in SAR research; it is used to interpret observed phenomena, to investigate new processing techniques, and is a key component of the model-based building reconstruction method.

In general, the simulation process consists of two stages. In the first stage, a scene is constructed and the reflectivity of the scene is calculated at discrete points using a 
model for electromagnetic reflectivity. The second stage is the calculation of the twodimensional SAR signal produced by the scene. Either the raw radar measurements can be simulated and then processed to form a SAR image [34, 33, 35, 36, 37], or the SAR image can be simulated directly $[21,38,39]$. In general, simulating the raw radar measurements provides a more realistic result [40,37] because the SAR processing effects are present in the final image.

The most straightforward and accurate method for simulating the measurements is to use the time-domain signal model directly $[41,36]$. This entails calculating the complex-valued signal for each point in the scene for each time sample of each radar pulse used to form the synthetic aperture. The advantage of this method is that the exact sensor trajectory is used, so that distortions in the final image caused by deviations in the sensor flight path are reproduced in the simulation [35]. The disadvantage is that the computational cost increases with the number of points evaluated and with the sampling rate of the SAR sensor. Using the time-domain method to simulate the response generated by a building for a high-resolution sensor requires a significant amount of computation.

An more efficient alternative to the time-domain method is the frequency-domain method presented in [42]. The method uses Fast Fourier Transforms (FFTs) and an approximation of the SAR system transfer function. The disadvantages of this method are that the formulation is only valid for a particular type of SAR, and arbitrary deviations from an ideal straight-line sensor trajectory cannot be accommodated. Later extensions to the method addressed these issues to some degree $[43,44,45]$, but the frequency domain method remains unsuitable for producing a simulation that can be matched to an arbitrary SAR image for building reconstruction. 
Here, a new simulation technique was developed that is as accurate as the timedomain method and requires less computation for simulating high-resolution SAR measurements of a scene containing a building. This new simulation method was used for model-based building reconstruction. Accuracy is important for this application because the goal of the matching process is to measure the similarity between an actual building and an hypothesized building indirectly by comparing images of these buildings. If the images contain differences other than those due to a mismatch between the buildings, then the matching process will be unreliable.

\subsection{Mutual Information as a Similarity Measure}

The model-based building reconstruction method uses some measure of the similarity between a simulation image and a SAR image. Mutual information, a measure from information theory [46], was first proposed as a similarity measure for images in 1995 by Viola [47]. The measure was then applied to the problem of multi-modal medical image registration $[48,49]$, where the goal is to find a transformation from one image's coordinate system to the other that aligns the two images. The mutual information similarity measure has become a standard for medical image registration [50]; a comprehensive survey of the modalities and implementations used can be found in $[51]$.

Mutual information has also been used as a similarity measure for remote sensing image registration $[52,53,54]$. The applications are similar to those in the medical imaging field: multi-modal registration of optical images with LiDAR images for enhanced information, and multi-temporal sequences of the same modality for change detection [55]. Mutual information as a similarity measure for SAR image registration 
was proposed in 2003 by Chen [52] and, independently, by Xie and Ulaby [56]. Since then, it has been applied to registering SAR images with optical images [57, 58, 59], to registering interferometric pairs of SAR images [60], and to registering SAR images with different polarizations $[61,62]$. The application of matching a SAR image with a simulation image, as in Brunner [21, 22], is a new application of mutual information that is closely related to Viola's original work; Viola proposed using mutual information to match an optical image to a model-based simulation.

Mutual information belongs to a class of image similarity measures known as intensitybased measures, as distinguished from feature-based methods. Intensity-based methods are independent of content and context, and do not require a feature detection step prior to registration. Other intensity-based similarity measures commonly used are normalized cross-correlation (NCC) and mean squared difference (MSD) [63]. These measures assume a linear relationship between the intensities of the compared images and therefore may not be suitable to matching a SAR image to a simulation image. The absolute intensities of a SAR image depend on many variables which are unknown, and it is difficult to reproduce the same intensities with a simulation. In [50], it is shown that mutual information and correlation-based similarity measures are mathematically equivalent under certain assumptions.

The performance of an image similarity measure can be characterized by its consistency and smoothness $[64,49]$. Consistency is important in the context of model-based reconstruction; the similarity measure used to match a simulation image to a SAR image must give the same result for different instances of the simulation image, and must give the same result for different SAR images of the same building. Smoothness was used by Brunner [22] as a criteria for accepting the building height hypothesis that maximized the mutual information between the SAR image and the simulation; 
if the mutual information as a function of the hypothesized height exhibited numerous or strong local maxima, then the result was rejected.

In this research, the consistency and smoothness of the mutual information measure was evaluated in the context of model-based building reconstruction. The evaluation was done using multiple SAR images of the same building and using multiple simulation instances of the same hypothetical building. 


\section{Chapter 3}

\section{Theoretical Background}

The purpose of this chapter is to provide the reader with the background - theory, terminology, notation - for understanding the details of this research. The chapter begins with an overview of electromagnetic theory that explains what is measured by a SAR sensor. The next section describes how SAR data is collected, and how the data is processed to form an image. This is important for understanding the image features produced by a building and for understanding how SAR signals can be simulated. The final section presents basic concepts from Information Theory that were applied to extract information from SAR data.

\subsection{Electromagnetic Theory}

SAR, and radar in general, works by transmitting an electromagnetic pulse and then measuring the electromagnetic energy reflected by illuminated surfaces. This research used electromagnetic theory to characterize the electromagnetic energy reflected by a building. 


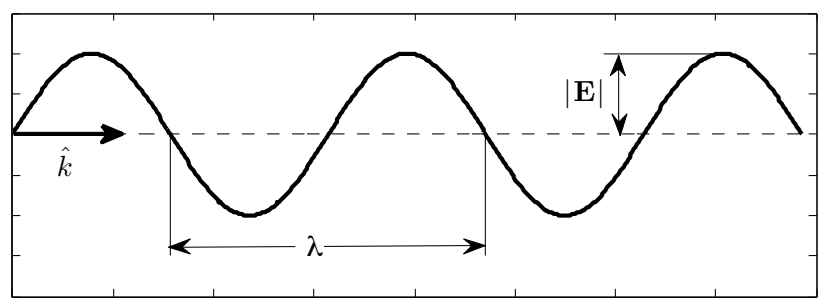

Figure 3.1: An electromagnetic wave propagates in a direction $\hat{\boldsymbol{k}}$ with wavelength $\lambda$.

An electric field $\mathbf{E}$ is a vector field having magnitude, phase and direction at every point in space. The relationships between electric fields, magnetic fields, charges and currents are governed by a set of physical laws known as Maxwell's Equations. Electromagnetic energy propagates through space in waves. The waves propagate at the speed of light in free space (air). The direction of propagation is denoted by unit vector $\hat{\boldsymbol{k}}$ and the wavelength is denoted by $\lambda$ as shown in Figure 3.1. The wavenumber of an electromagnetic wave is the spatial frequency of the wave, $k=2 \pi / \lambda$ in radians per meter. The polarization of a wave refers to the orientation of the wave in space: if the propagation direction defines the $y$-axis in a three-dimensional coordinate system, then a wave oscillating in the $\mathrm{y}-\mathrm{z}$ plane is vertically polarized and a wave oscillating in the $\mathrm{x}-\mathrm{y}$ plane is horizontally polarized.

The term scattering is used to describe the interaction of an electromagnetic wave, or field, with an object. If a source generates an electric field in an unbounded, homogenous space, the total field at some observation point is equal to the sourcegenerated field. If an object is introduced into the space, the difference between the total field and the source-generated field is the scattered field. The source-generated field is called the incident field, and the object is called the scatterer. If the object is a good conductor, the incident field induces a current on the surface of the object. The current then gives rise to a radiated electromagnetic field, which is the scattered field. Radar works by transmitting an electromagnetic wave (the incident field), and 


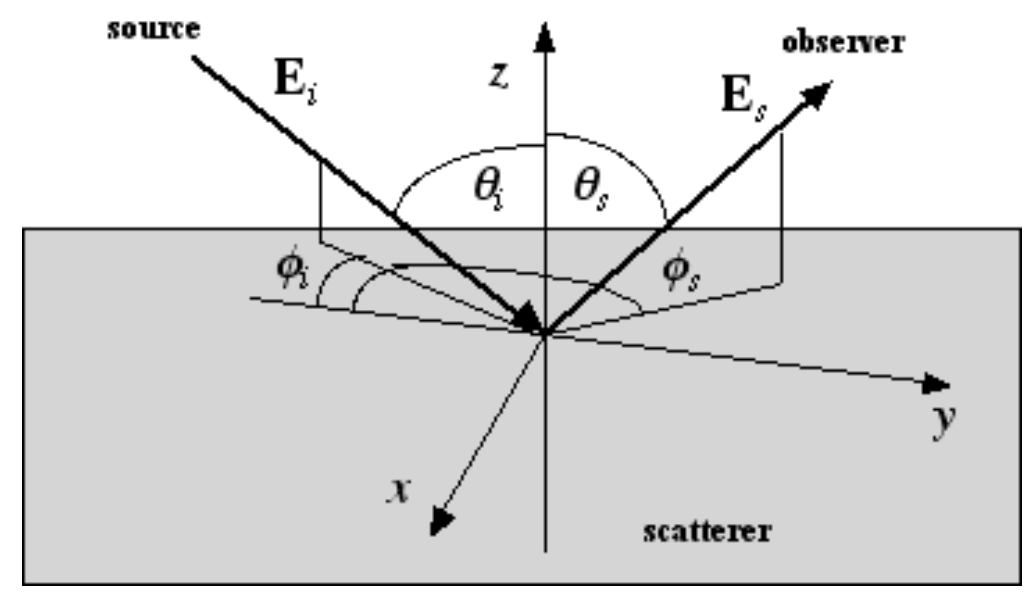

Figure 3.2: The incident field $\mathbf{E}_{i}$ emanating from a source and the scattered field $\mathbf{E}_{s}$ observed at some point in space. The arrows from the source to the scatterer and from the scatterer to the observer indicate the propagation direction of the electromagnetic waves.

then measuring the scattered field produced by objects in the illuminated space.

The geometry used to describe incidence and scattering are illustrated in Figure 3.2. The angles $\theta_{i}$ and $\phi_{i}$ are the angles of incidence and describe the position of the source relative to the scatterer. The angles $\theta_{s}$ and $\phi_{s}$ are the angles that describe the position at which the scattered field is observed (measured). When the source and observation point are colocated, the observed scattering is called monostatic; if the scattering is observed at a location different from the source, it is termed bistatic. For monostatic scattering, $\theta_{s}=\theta_{i}$ and $\phi_{s}=\phi_{i}$. SAR is generally monostatic, with the transmitter and receiver at the same antenna. The propagation vector of the incident field is

$$
\hat{\mathbf{k}}=\sin \phi_{i} \sin \theta_{i} \overrightarrow{\boldsymbol{x}}+\cos \phi_{i} \sin \theta_{i} \overrightarrow{\boldsymbol{y}}+\cos \theta_{i} \overrightarrow{\boldsymbol{z}}
$$

The radar cross section (RCS) of an object is a measure of the object's "scattering power". It is defined in terms of a power ratio [65]: 


$$
\operatorname{RCS}\left(\theta_{s}, \phi_{s} ; \theta_{i}, \phi_{i}\right)=\lim _{r \rightarrow \infty} 4 \pi r^{2} \frac{\left|\mathbf{E}_{s}\right|^{2}}{\left|\mathbf{E}_{i}\right|^{2}}
$$

where $\mathbf{E}_{i}$ is the incident field, $\mathbf{E}_{s}$ is the scattered field, and $r$ is the distance between the scatterer and the observer. The measure is usually normalized to unit area of one square meter and given in $\mathrm{dB}$.

The strength of the scattered field depends on the angles of incidence and scattering, and on the radiometric properties of the object. The radiometric properties of a material relevant to radar are its electrical permittivity and its surface roughness. The permittivity is frequency-dependent and indicates a material's ability to store electrical energy; it is usually given as relative permittivity $\epsilon_{r}$, a complex value, which is the permittivity relative to the permittivity of air. The roughness of a surface refers to wavelength scale deviations in the height of the surface from a smooth, flat plane.

A smooth, flat surface reflects energy in one direction called the specular, according to Snell's Law: the specular reflection travels away from the point of incidence on the surface at an angle equal to the incident angle. This means that the total energy of the scattered field is concentrated in the direction $\theta_{s}=\theta_{i}, \phi_{s}=\phi_{i}+\pi$ and is very weak or non-existant elsewhere. If the surface is rough, the reflected energy is more spread out in all directions. The scattering from a rough surface is important for monostatic radar systems, such as SAR, because the roughness of surfaces causes energy to be scattered back toward the sensor.

Several methods have been established for calculating the scattered field, and depending on the problem and the desired accuracy of the results, one method may be more approprate than another. Two methods commonly used for radar scattering 
problems are Physical Optics and Geometric Optics. The following short descriptions are based on Balanis [66].

Geometric Optics (GO), also called ray optics or ray tracing, is appropriate for highfrequency problems where the scattering in the specular direction is dominant, i.e. smooth surfaces with a large radius of curvature. The advantage of this method is that its application is straightforward.

Physical Optics (PO) approximates the induced surface current of an object using an infinite, flat, perfect conductor. The scattered field can then be found using radiation integral equations. The integration is usually only performed over the unshadowed regions of the surface, and edge effects are not included. This method is most appropriate for objects whose dimensions are much greater than a wavelength (electrically large) and whose surface contour is simple.

The PO model for the monostatic scattering from a rectangular plate is [25]

$$
\mathbf{E}_{s}=\frac{\mathrm{j} k \exp [j k r]}{4 \pi r} \mathbf{S}\left(\hat{\boldsymbol{n}}, \hat{\boldsymbol{k}}, \epsilon_{r}\right)\left|\mathbf{E}_{i}\right| I_{s}\left(a, b, k, \theta_{i}, \phi_{i}\right)
$$

where 
$k(\mathrm{rad} / \mathrm{m})$ is the wavenumber of the incident field,

$r(\mathrm{~m}) \quad$ is the distance between the source of the incident field and the rectangular surface, $\hat{\boldsymbol{n}} \quad$ is the normal vector of the surface,

$\hat{\boldsymbol{k}} \quad$ is the propagation direction vector of the incident field,

$\epsilon_{r} \quad$ is the relative permittivity of the surface,

$a, b(\mathrm{~m}) \quad$ are the length and width of the surface,

$\theta_{i} \quad$ is the angle at which the incident field illuminates the surface, and

$\phi_{i} \quad$ is the rotation angle in the plane of the surface.

The term $\mathbf{S}(\cdot)$ is the polarization matrix which projects the scattered field into the principle scattering plane for the four different source-receiver polarization combinations. The LiMIT sensor is co-polarized; the source and receiver are both horizontally polarized so only the $S_{h h}$ element of the matrix is needed,

$$
\begin{aligned}
S_{h h}\left(\hat{\boldsymbol{n}}, \hat{\boldsymbol{k}}, \epsilon_{r}\right)= & -\left(\hat{\boldsymbol{e}}_{h} \cdot \hat{\boldsymbol{q}}\right)(\hat{\boldsymbol{n}} \cdot \hat{\boldsymbol{k}}) \hat{\boldsymbol{q}}\left(1-R_{\perp}\right)+\left(\hat{\boldsymbol{e}}_{h} \cdot \hat{\boldsymbol{p}}\right)(\hat{\boldsymbol{n}} \times \hat{\boldsymbol{q}})\left(1+R_{\|}\right) \\
& +\left(\hat{\boldsymbol{e}}_{h} \cdot \hat{\boldsymbol{q}}\right)(-\hat{\boldsymbol{k}} \times(\hat{\boldsymbol{n}} \times \hat{\boldsymbol{q}}))\left(1+R_{\perp}\right) \\
& +\left(\hat{\boldsymbol{e}}_{h} \cdot \hat{\boldsymbol{p}}\right)(\hat{\boldsymbol{n}} \cdot \hat{\boldsymbol{k}})(-\hat{\boldsymbol{k}} \times \hat{\boldsymbol{q}})\left(1-R_{\|}\right)
\end{aligned}
$$

where $\hat{\boldsymbol{e}}_{h}$ is the horizontal polarization vector, $\hat{\boldsymbol{p}}$ and $\hat{\boldsymbol{q}}$ are the local horizontal and vertical polarization vectors at the surface of the plate. The terms $R_{\perp}$ and $R_{\|}$are the Fresnel reflection coefficients,

$$
\begin{aligned}
& R_{\perp}=\frac{\cos \theta_{i}-\sqrt{\epsilon_{r}-\sin ^{2} \theta_{i}}}{\cos \theta_{i}+\sqrt{\epsilon_{r}-\sin ^{2} \theta_{i}}} \\
& R_{\|}=\frac{\epsilon_{r} \cos \theta_{i}-\sqrt{\epsilon_{r}-\sin ^{2} \theta_{i}}}{\epsilon_{r} \cos \theta_{i}+\sqrt{\epsilon_{r}-\sin ^{2} \theta_{i}}}
\end{aligned}
$$


The term $I_{s}(\cdot)$ is the scattering pattern for a rectangular surface,

$$
\begin{aligned}
I_{s}\left(a, b, k, \theta_{i}, \phi_{i}\right)= & \exp \left[\mathrm{j} k a \cos \theta_{i}\right] \exp \left[-\mathrm{j} k b \sin \theta_{i}\right] \\
& a b \cos \phi_{i} \operatorname{sinc}\left[-k b \sin \theta_{i}\right] \operatorname{sinc}\left[k a \sin \phi_{i} \cos \theta_{i}\right]
\end{aligned}
$$

Substituting (3.3), (3.7) and (3.4) into (3.2) gives

$$
\begin{aligned}
\operatorname{RCS}\left(\theta_{i}, \phi_{i}\right) & =\frac{k^{2}}{4 \pi}\left|S_{h h}\right|^{2}\left|I_{s}\right|^{2} \\
& =\frac{k^{2}}{4 \pi}\left|S_{h h}\right|^{2}(a b)^{2} \cos ^{2} \phi_{i} \operatorname{sinc}^{2}\left[-k b \sin \theta_{i}\right] \operatorname{sinc}^{2}\left[k a \sin \phi_{i} \cos \theta_{i}\right]
\end{aligned}
$$

which is the PO model for the monostatic RCS of a rectangular surface. The PO model assumes a smooth surface; roughness can be accounted for by incorporating the surface height statistics in the scattering pattern $I_{s}$ [67].

The PO and GO models can be used to calculate the scattered field from a single surface. Buildings are composed of multiple surfaces, such as vertical walls and roof sections. The total scattered field for the entire structure is the sum of the scattering from each of the component surfaces. For a structure such as a dihedral or trihedral, which could be formed by a building's vertical walls and the ground, the scattered field from one surface becomes an incident field on another surface. The procedure to determine the total scattered field is to decompose the scattering into single bounce, double bounce, triple bounce, etc. components $[68,69]$. A bounce refers to a ray bouncing off a surface, or a wave being reflected. The mulitple bounce mechanism is illustrated in Figure 3.3. For each bounce,

1. Use GO or PO to derive the incident magnetic field $\mathbf{H}_{i}$ on the first surface $S^{\prime}$, 


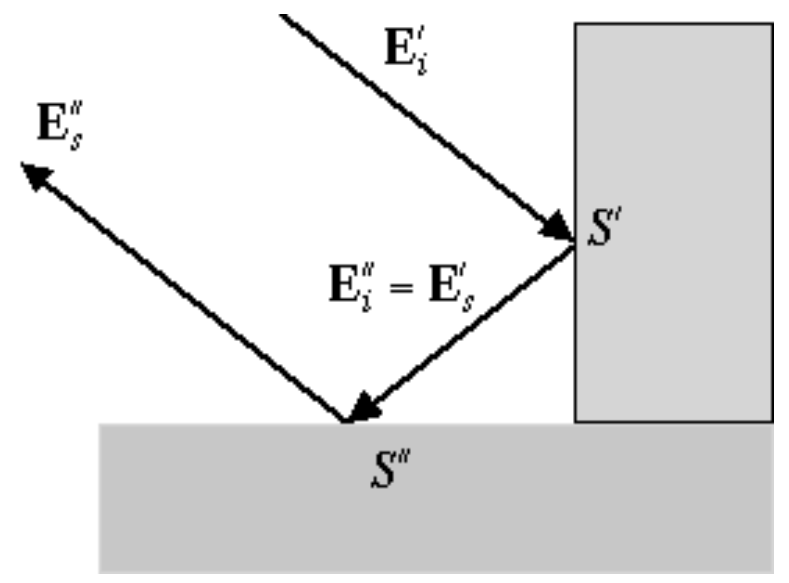

Figure 3.3: The scattering from a dihedral.

given the incident electric field $\mathbf{E}_{i}$.

2. Use the PO approximation to find the induced current $\mathbf{J}_{S^{\prime}}=2 \hat{n} \times \mathbf{H}_{i}$, where $\hat{n}$ is the normal to the surface.

3. Derive $\mathbf{E}_{s}$ from $\mathbf{J}_{S^{\prime}}$ using the radiation pattern $I_{s}$, incorporating surface roughness, if appropriate.

4. Repeat for each additional surface, using the scattered field $\mathbf{E}_{s}$ from the previous surface as the incident field $\mathbf{E}_{i}$ on the next surface.

The total scattered field for the entire structure is the sum of the scattering from each of the components.

\subsection{Spotlight Synthetic Aperture Radar}

A SAR image is a spatial map of the scattering intensity produced by the imaged scene. The total scattered field in response to a single transmitted pulse is the superposition of the scattering from the entire scene; the SAR process employs multiple 
pulses and subsequent signal processing to resolve the spatial location of scatterers in the scene. The type of SAR described here is called spotlight SAR. Other types of SAR include strip-map mode, polarimetric and interferometric SAR. For spotlight SAR, the radar sensor (transmitter and receiver) is mounted on a moveable platform, such as an aircraft, a satellite, or a mechanical boom. The sensor is mounted so that it looks in a direction orthogonal to the direction of platform motion. The platform moves along a trajectory, and at regularly spaced intervals, the sensor transmits an electromagnetic pulse and samples the resulting scattered field. Each pulse is aimed at the center of the scene to be imaged, and together the pulses are processed to form a synthetic aperture.

At this point, it is useful to define some common terminology with the aid of Figure 3.4. The portion of the platform trajectory used to synthesize the aperture forms a coherent processing interval (CPI). The area illuminated by the radar is referred to as a scene. The center of the scene is called the aimpoint because it is the point at which the sensor is aimed. This point is chosen as the origin of the coordinate system constructed to define the geometry of the SAR CPI. The look direction of the sensor is called range, and the platform motion direction is called cross-range. For spotlight mode SAR, the platform trajectory is ideally a straight line. The plane containing the trajectory and the aimpoint is called the slant plane. The grazing angle is the angle between the ground plane and the slant plane. The view angle is measured in the ground plane with reference to the vector from the aimpoint to the center of the synthetic aperture projected onto the ground plane.

The scene is a surface in three-dimensional space, but since a single SAR data collection is only capable of resolving location in two dimensions (range and crossrange), a reference plane is chosen for mapping the results of SAR processing to physical space. 
This plane is called the processing plane, and it is usually chosen to be the slant plane or the ground plane.

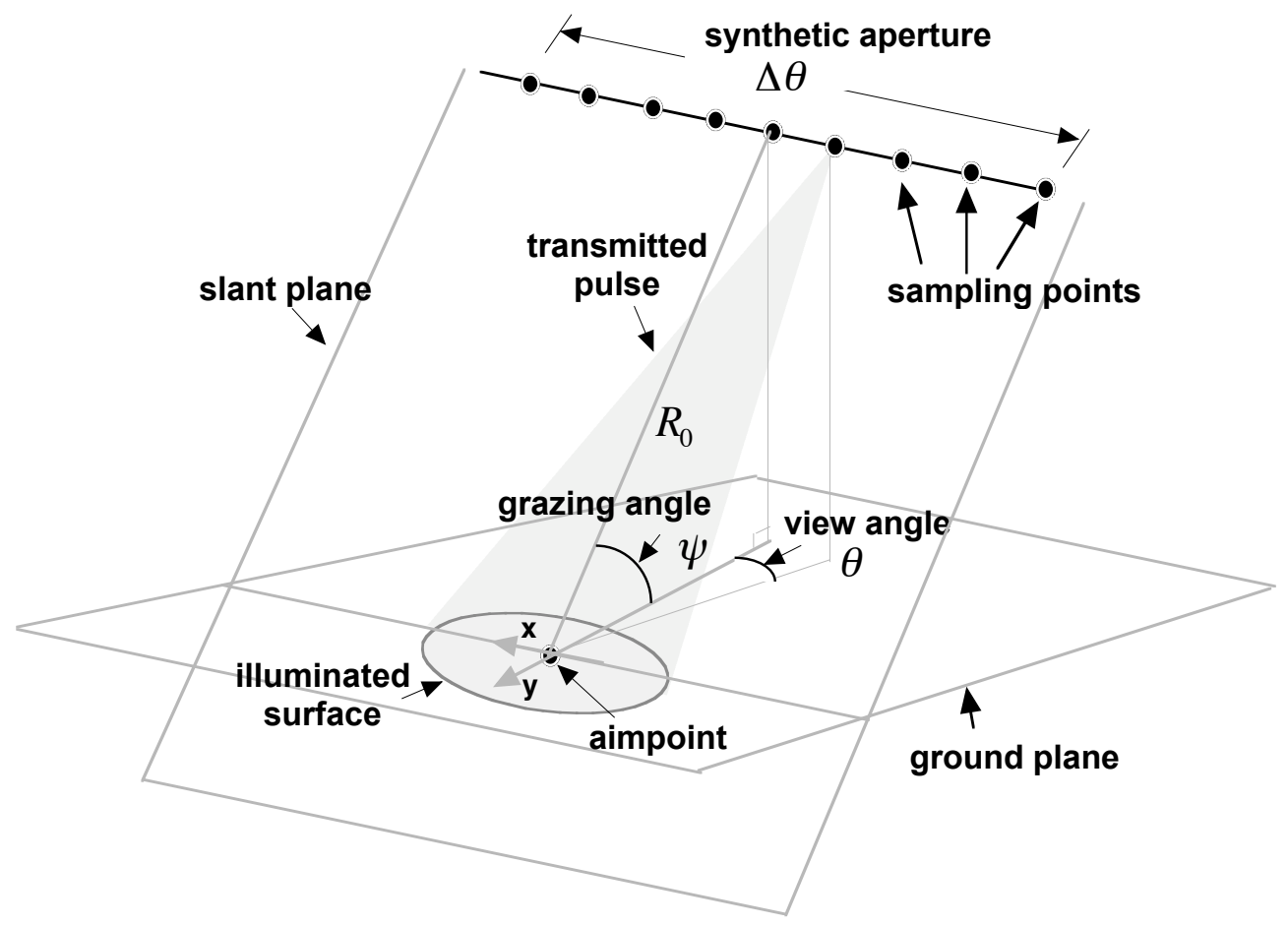

Figure 3.4: SAR spotlight mode data collection.

The received signal from a single pulse can resolve the location of objects in one dimension only, range, using the relation $r=c t / 2$, where $r$ is the range between the sensor and the reflecting object, $c$ is the speed of light and $t$ is the time elapsed between the pulse transmission and the reception of the echo. To resolve the location of objects in the cross-range dimension, a very narrow beam could be used to illuminate one narrow strip of the scene at a time. The width of the illuminated strip on the ground is related to the antenna size by:

$$
D=r \frac{\lambda}{L}
$$

where $r$ is the range (stand-off distance) from the antenna to the ground, $\lambda$ is the wavelength of the pulse and $L$ is the length of the physical antenna aperture in cross- 
range. To achieve a fine cross-range resolution using this method would require an impractically large antenna. For example, to achieve one meter resolution using a $10 \mathrm{GHz}$ radar (3 $\mathrm{cm}$ wavelength) from an airborne platform with a $10 \mathrm{~km}$ standoff distance would require a 30 meter antenna. The synthetic aperture overcomes the limitation of the physical aperture by synthesizing an aperture from multiple measurements along the platform trajectory. The cross-range resolution of SAR data is inversely proporional to the length of the synthetic aperture and is independent of the physical antenna size and the stand-off distance. The physical antenna still determines the cross-range width of the illuminated area, so a small antenna with a wide beam becomes desirable. In this way, SAR is capable of imaging a large area with high resolution from a long distance.

To understand how a scene is imaged by the SAR sensing process, it is useful to first consider the signal received in response to a single pulse and then to look at how the signals from all the pulses are synthesized into an aperture and processed to form an image.

The transmitted pulse is a linear frequency modulated (FM) signal, known as a chirp:

$$
p(t)=\exp \left[\mathrm{j}\left(\omega_{0} t+\alpha t^{2}\right)\right] \operatorname{rect}\left[\frac{t}{T}\right],
$$

where $\omega_{0}$ is the center frequency of the chirp in radians per second, $2 \alpha$ is the chirp rate in radians per second squared, and $T$ is the pulse duration in seconds. The bandwidth of the chirp, in $\mathrm{Hz}$, is

$$
\beta=\frac{2 \alpha T}{2 \pi}
$$


Consider the received echo from a single point in the scene in response to the $m$ th pulse. Let the point be at a location in the slant plane given by $\overrightarrow{\boldsymbol{r}}$ and let the point have a reflectivity of $\gamma$. The reflectivity has a magnitude that is the radar cross section of the point and a phase that is random (the random phase will be treated later). Let the sensor be at position $\overrightarrow{\boldsymbol{R}}_{m}$ along the platform trajectory and the distance between the point and the sensor be

$$
r=\left|\overrightarrow{\boldsymbol{R}}_{m}-\overrightarrow{\boldsymbol{r}}\right|
$$

The received echo is a time-shifted, amplitude-scaled version of the transmitted pulse. This received signal is mixed down to baseband (shifted in frequency by $-\omega_{0}$ ) and sampled at discrete times $t_{n}$ beginning after some delay from the center time of the transmitted pulse. The raw recorded signal is then

$$
\begin{aligned}
s_{m}\left(t_{n}\right) & =\gamma p\left(t_{n}-\frac{2}{c}\left|\overrightarrow{\boldsymbol{R}}_{m}-\overrightarrow{\boldsymbol{r}}\right|\right) \exp \left[-\mathrm{j} \omega_{0} t_{n}\right] \\
& =\gamma \exp \left[-\mathbf{j} \omega_{0} \frac{2}{c} r\right] \exp \left[\mathbf{j} \alpha\left(t_{n}-\frac{2}{c} r\right)^{2}\right] \operatorname{rect}\left[\frac{t_{n}-\frac{2}{c} r}{T}\right]
\end{aligned}
$$

where $c$ is the speed of light, and $\frac{2}{c} r$ is the time delay between the transmitted pulse and the received echo. At the end of the collection, the stored signals from all the pulses of the CPI comprise the SAR raw data.

The raw data is then processed to form an image. The processing proceeds as follows. The signal from each pulse is transformed into the frequency domain and then multiplied by a filter matched to the transmitted pulse in order to demodulate the 
signal. The transform of the pulse return signal is

$$
\begin{aligned}
S_{m}(\omega) & =\gamma \exp \left[-\mathrm{j} \omega_{0} \frac{2}{c} r\right] \exp \left[-\mathrm{j}\left(\frac{\omega}{2 \alpha}\right)^{2}\right] \exp \left[-\mathrm{j} \omega \frac{2}{c} r\right] \operatorname{rect}\left[\frac{\omega}{2 \alpha T}\right] \\
& =\gamma \exp \left[-\mathrm{j}\left(\omega_{0}+\omega\right) \frac{2}{c} r\right] \exp \left[-\mathrm{j}\left(\frac{\omega}{2 \alpha}\right)^{2}\right] \operatorname{rect}\left[\frac{\omega}{2 \alpha T}\right] .
\end{aligned}
$$

Multiplication by a matched filter in the frequency domain is equivalent to correlation in the time domain with a reference signal that is the inverse transform of the matched filter. The reference signal is a time-shifted baseband version of the transmitted pulse,

$$
\begin{aligned}
p_{0}\left(t_{n}\right) & =\exp \left[\mathrm{j} \omega_{0} t_{n}\right] p\left(t_{n}-\frac{2}{c} r_{0}\right) \\
& =\exp \left[-\mathrm{j} \omega_{0} \frac{2}{c} r_{0}\right] \exp \left[\mathrm{j} \alpha\left(t_{n}-\frac{2}{c} r_{0}\right)^{2}\right] \operatorname{rect}\left[\frac{t_{n}-\frac{2}{c} r_{0}}{T}\right]
\end{aligned}
$$

where $r_{0}$ is the distance between the center of the scene and the center of the portion of the flight trajectory used for the CPI. The matched filter is the complex conjugate of the transform of the reference signal, phase shifted to account for the actual distance between the center of the scene and the sensor position $\overrightarrow{\boldsymbol{R}}_{m}$.

The transform of the reference signal is

$$
P_{0}(\omega)=\exp \left[-\mathrm{j}\left(\omega_{0}+\omega\right) \frac{2}{c} r_{0}\right] \exp \left[-\mathrm{j}\left(\frac{\omega}{2 \alpha}\right)^{2}\right] \operatorname{rect}\left[\frac{\omega}{2 \alpha T}\right]
$$

The demodulated signal is then

$$
\begin{aligned}
F_{m}(\omega) & =S_{m}(\omega) P_{0}^{*}(\omega) \exp \left[-\mathrm{j}\left(\omega_{0}+\omega\right) \frac{2}{c}\left(r_{0}-R_{m}\right)\right] \\
& =\gamma \exp \left[\mathrm{j}\left(\omega_{0}+\omega\right) \frac{2}{c}\left(R_{m}-r\right)\right] \operatorname{rect}\left[\frac{\omega}{2 \alpha T}\right]
\end{aligned}
$$




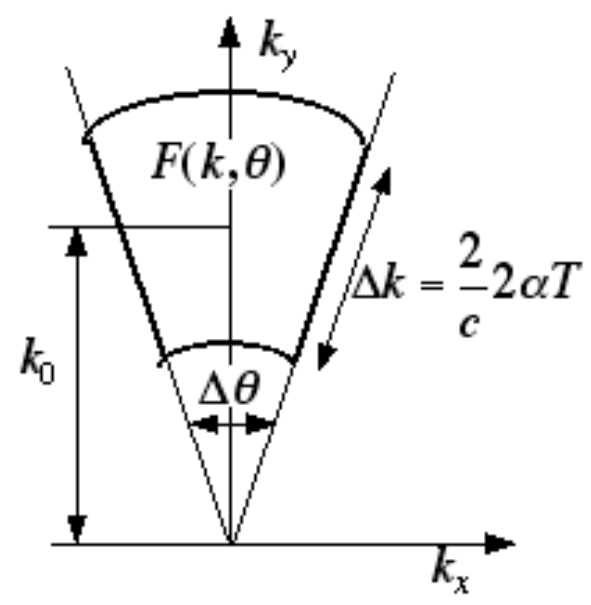

Figure 3.5: The phase history exists in the spatial frequency domain. The demodulated returns from each pulse lie along lines of constant $\theta$ corresponding to the view angle of the pulse.

The collection of all the demodulated signals from all the pulses is called the phase history. The phase of the signals contains information about the spatial location of the source of reflected energy $\gamma$. Each filtered signal corresponds to a view angle $\theta$, as shown in Figure 3.4. The phase history is the two-dimensional transform of the imaged scene reflectivity; in this case the scene reflectivity is a delta function since only a single point is being considered. To make this more clear, a change of variable is introduced,

$$
k=\left(\omega_{0}+\omega\right) \frac{2}{c}
$$

where $k$ is spatial frequency in radians per meter. (Note that this definition of $k$ is twice the wavenumber $k$ defined in the previous section; the factor of two comes from the two-way travel time of the signal.) The phase history is then expressed, using (3.17), as

$$
F\left(k, \theta_{m}\right)=\gamma \exp \left[\mathrm{j} k\left(R_{m}-r\right)\right] \operatorname{rect}\left[\frac{k-k_{0}}{4 \alpha T / c}\right]
$$

The phase history is a two-dimensional surface in the spatial frequency domain as illustrated in Figure 3.5. 
To relate the phase history to the spatial domain, let the polar slant plane coordinates of the sensor at $\overrightarrow{\boldsymbol{R}}_{m}$ be $\left[R_{m}, \theta_{m}\right]$ and of the point at $\overrightarrow{\boldsymbol{r}}$ be $[\rho, \varphi]$. Then by the Law of Cosines

$$
\left|\overrightarrow{\boldsymbol{R}}_{m}-\overrightarrow{\boldsymbol{r}}\right|^{2}=R_{m}^{2}+\rho^{2}-2 \rho R_{m} \sin \left(\theta_{m}+\varphi\right) .
$$

Since $\rho \ll R_{m}$,

$$
r=\left|\overrightarrow{\boldsymbol{R}}_{m}-\overrightarrow{\boldsymbol{r}}\right| \approx R_{m}-\rho \sin \left(\theta_{m}+\varphi\right)+\frac{\rho^{2}}{2 R_{m}} \cos ^{2}\left(\theta_{m}+\varphi\right) .
$$

Then, using (3.19) and (3.21), the phase history is

$$
\begin{aligned}
F(k, \theta)= & \gamma \exp \left[\mathrm{j} k\left(\rho \sin \left(\theta_{m}+\varphi\right)-\frac{\rho^{2}}{2 R_{m}} \cos ^{2}\left(\theta_{m}+\varphi\right)\right)\right] \\
& \times \operatorname{rect}\left[\frac{k-k_{0}}{4 \alpha T / c}\right] \\
= & \gamma \operatorname{rect}\left[\frac{k-k_{0}}{4 \alpha T / c}\right] \exp \left[\mathrm{j} k \rho \sin \left(\theta_{m}+\varphi\right)\right] \\
& \times \exp \left[-\mathrm{j} \frac{k \rho^{2}}{2 R_{m}} \cos ^{2}\left(\theta_{m}+\varphi\right)\right] .
\end{aligned}
$$

The signal of (3.22) is a two-dimensional rectangular pulse, scaled by the reflectivity of the point and phase shifted by two exponentials. The rect function can be approximated as the product of two rect functions in the Cartesian coordinates shown in Figure 3.5,

$$
\operatorname{rect}\left[\frac{k-k_{0}}{4 \alpha T / c}\right] \approx \operatorname{rect}\left[\frac{k_{x}}{k_{0} \Delta \theta}\right] \operatorname{rect}\left[\frac{k_{y}-k_{0}}{4 \alpha T / c}\right],
$$

where $k_{x}=k \sin \theta_{m} \approx k_{0} \theta$ and $k_{y}=k \cos \theta_{m} \approx k$. 
The phase of the first exponential is a linear shift that corresponds to a shift in the spatial domain,

$$
\begin{aligned}
k \rho \sin \left(\theta_{m}+\varphi\right) & =k \rho\left(\sin \theta_{m} \cos \varphi+\cos \theta_{m} \sin \varphi\right) \\
& =\left(k \sin \theta_{m}\right)(\rho \cos \varphi)+\left(k \cos \theta_{m}\right)(\rho \sin \varphi) \\
& =k_{x} \hat{x}+k_{y} \hat{y}
\end{aligned}
$$

$\hat{x}$ and $\hat{y}$ are the Cartesian slant plane coordinates of the point $\overrightarrow{\boldsymbol{r}}$.

The second exponential is a distortion called the range curvature effect. Ignoring this distortion, a two-dimensional inverse transform into the spatial domain produces the intensity image,

$$
|f(\tilde{x}, \tilde{y})|=|\gamma| \operatorname{sinc}\left[\frac{k_{0} \Delta \theta}{2 \pi}(\tilde{x}-\hat{x})\right] \operatorname{sinc}\left[\frac{4 \alpha T / c}{2 \pi}(\tilde{y}-\hat{y})\right]
$$

where $\tilde{x}$ and $\tilde{y}$ are image coordinates. Equation (3.25) is the SAR image of a single point. This is referred to as the point spread function. It shows how the resolution of the image is determined by the span of the synthetic aperture $\Delta \theta$ and the bandwidth $\beta$ of the transmitted pulse,

$$
\begin{aligned}
& \rho_{x}=\frac{2 \pi}{k_{0} \Delta \theta}=\frac{\lambda}{2 \Delta \theta}, \\
& \rho_{y}=\frac{2 \pi}{4 \alpha T / c}=\frac{c}{2 \beta},
\end{aligned}
$$

where $\rho_{x}$ is the cross-range resolution and $\rho_{y}$ is the range resolution.

Two effects were ignored in the development of 3.25, the range curvature effect and the effect of the random phase contained in the reflectivity $\gamma$. The first effect causes a slight defocusing in the image. The second effect is what produces the speckle noise 
characteristic of SAR imagery.

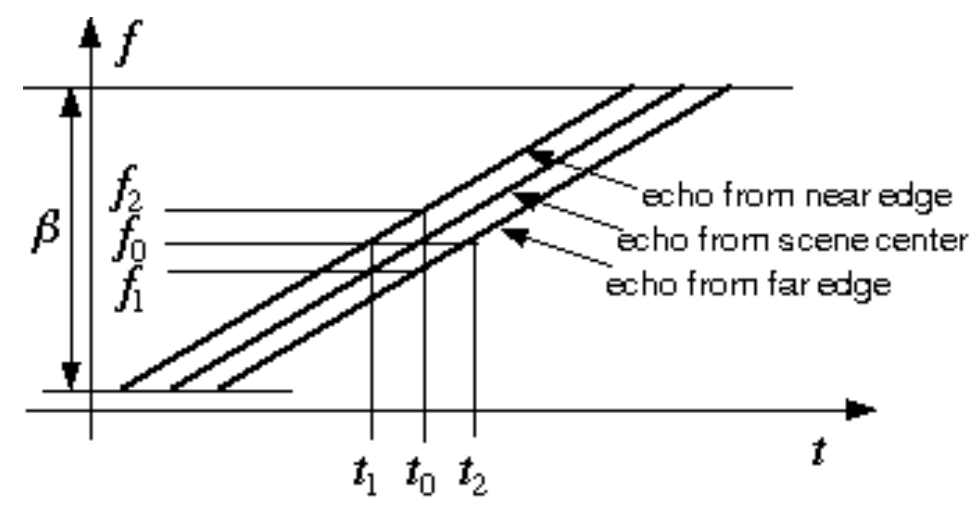

Figure 3.6: Time-frequency diagram of the echoes from a single transmitted pulse. The time delay of each echo is proportional to distance traveled, $t=(2 / c) r$, where $c$ is the speed of light. The instantaneous frequency of each echo at time $t_{0}$ is $f_{0}+2 \alpha\left(t-t_{0}\right) /(2 \pi)$.

The preceding theoretical development of how an image is formed from the SAR data used a single point of reflectivity in the scene for simplicity. In reality, the return signal from each pulse is the superposition of the echos from all points in the scene. Figure 3.6 shows a time-frequency diagram of echoes from the near edge, far edge and center of the scene. Each echo exists for a duration $T$, the duration of the chirp, and is shifted in time proportionally to the distance travelled. The bandwidth of the total signal produced by the scene at a given point in time is

$$
\begin{aligned}
\beta_{s} & =f_{2}-f_{1} \\
& =\frac{2 \alpha}{2 \pi} \frac{2}{c}\left(r_{2}-r_{1}\right),
\end{aligned}
$$

where $r_{1}$ is the distance to the near edge of the scene and $r_{2}$ is the distance to the far edge of the scene. This means that the bandwidth of the signal containing information about the illuminated scene is determined by the length of the scene in the range dimension. For spotlight mode SAR, the time duration of the chirp is 
chosen so that

$$
\beta_{s} \ll \beta
$$

This property of the SAR raw signal is exploited in the new efficient SAR simulation method developed for this research. The simulation method, described in Chapter 5 , is based on the equations presented here for the raw recorded signal (3.13), the matched filter (3.16), and the phase history (3.22). 

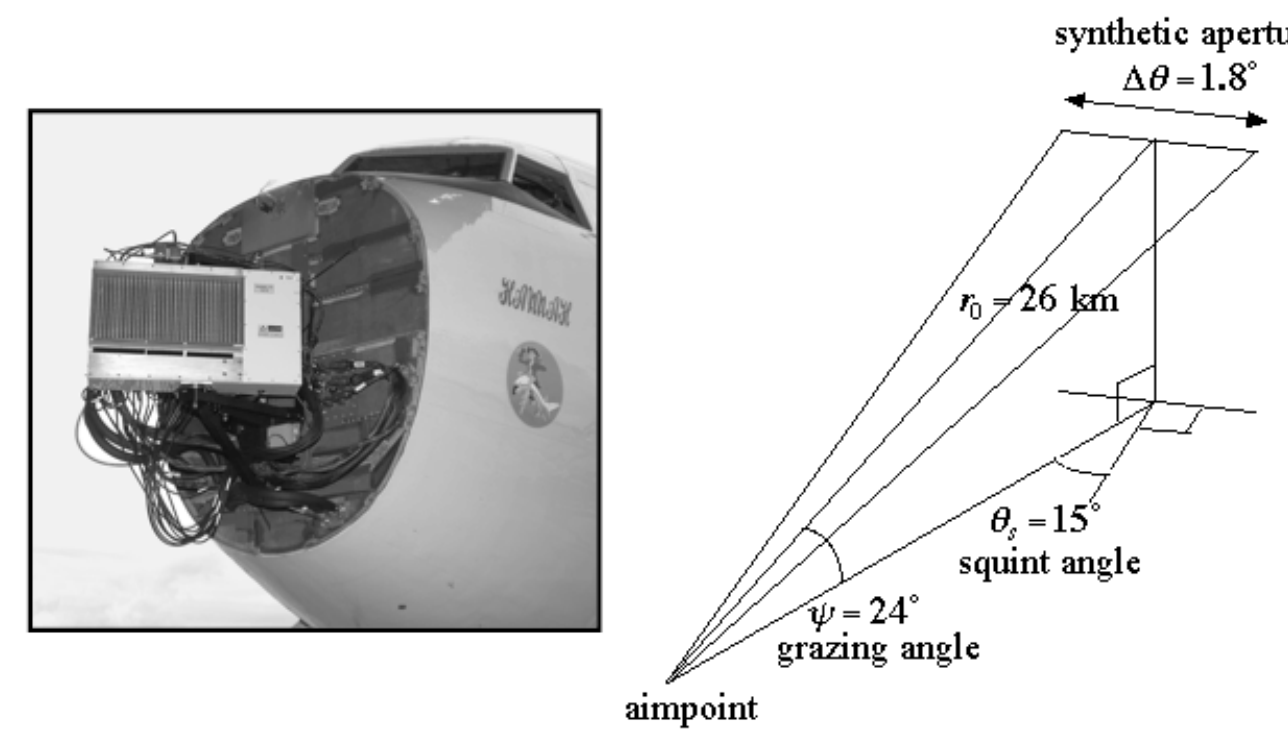

a) LiMIT sensor.

b) Typical LiMIT geometry.

Figure 3.7: The LiMIT sensor (a) is an 8-channel array mounted on the nose of a modified Boeng 707. The exact geometry (b) for each CPI is calculated from the recorded sensor positions.

\subsubsection{The LiMIT SAR Sensor}

The data used for this research were collected by the MIT-Lincoln Laboratory SAR sensor, LiMIT. The LiMIT SAR sensor is an 8-channel electronically steered array antenna mounted on a modified Boeing 707. The sensor is mounted on the nose of the plane, aimed to the left side and pointed slightly forward in what is known as a squinted configuration, shown in Figure 3.7a. The typical LiMIT collection geometry is shown in Figure 3.7b. Onboard, the analog signal from each channel is mixed down in frequency, low-pass filtered and sampled. The sampled signal is recorded as 8-bit signed integers. The LiMIT dataset used for this research was collected during a test mission at San Clemente Island, CA.

The data for each CPI consists of the raw recorded signal $s_{m}(t)$, the reference signal 

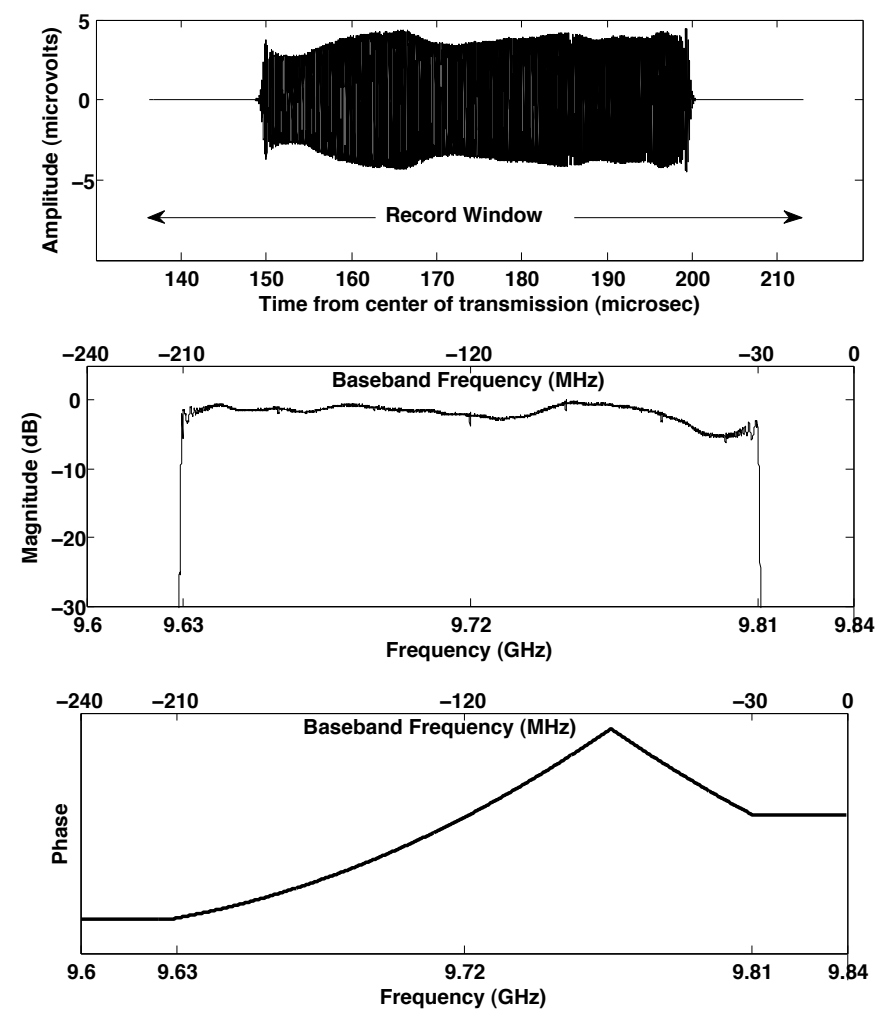

Figure 3.8: The LiMIT reference signal $p_{0}(t)$ is the transmitted waveform recorded in the center of the record window (top). The magnitude (center) and phase (bottom) of the Fourier transform of the recorded pulse are shown. The onboard mixdown process shifts the spectrum from the original transmission band centered at $9.72 \mathrm{GHz}$ to the band centered at $-120 \mathrm{MHz}$.

$p_{0}(t)$ and the sensor positions $\overrightarrow{\boldsymbol{R}}_{m}$. Figure 3.8 shows the time-domain waveform and the frequency domain magnitude and phase of the reference signal. The parameters of the LiMIT sensor are summarized in Table 3.1. Example images from the dataset are shown in Figure 3.9 and an optical image of the scene is included for comparison. The images were formed from the raw recorded signals using code provided by MIT Lincoln Laboratory. 
Table 3.1: LiMIT Parameters

\begin{tabular}{|c|c|c|}
\hline Parameter & Symbol & Value \\
\hline \multicolumn{3}{|c|}{ Physical Aperture } \\
\hline Horizontal Length & & $0.4826 \mathrm{~m}$ (19 in.) \\
\hline Vertical Length & & $0.1778 \mathrm{~m}(7$ in. $)$ \\
\hline Beamwidth in cross-range & & $2.7 \mathrm{~km}$ \\
\hline Beamwidth in range & & $4.8 \mathrm{~km}$ \\
\hline \multicolumn{3}{|c|}{ Synthetic Aperture } \\
\hline Number of pulses & $N_{p}$ & 9072 \\
\hline Pulse Repition Interval & PRI & $500 \mu \mathrm{sec}$ \\
\hline Angular span of aperture & $\Delta \theta$ & $1.57 \mathrm{deg}$ \\
\hline Length of aperture & $L_{s a}$ & $822 \mathrm{~m}$ \\
\hline \multicolumn{3}{|c|}{ Transmitted Waveform } \\
\hline Center frequency & $f_{0}$ & $9.72 \mathrm{GHz}$ \\
\hline Chirp rate & $2 \alpha$ & $-3.6 \mathrm{MHz} / \mu \mathrm{sec}$ \\
\hline Pulse duration & $T$ & $50 \mu \mathrm{sec}$ \\
\hline Pulse bandwidth & $\beta$ & $180 \mathrm{MHz}$ \\
\hline \multicolumn{3}{|c|}{ Recorded Signal } \\
\hline Record window duration & $T_{r}$ & $76 \mu$ sec \\
\hline Delay to center of record window & $t_{d}$ & $0.199498 \mu \mathrm{sec}$ \\
\hline Number of samples for each pulse & $N_{t}$ & 36800 \\
\hline Sampling rate & $f_{s}$ & $480 \mathrm{MHz}$ \\
\hline Sampling interval & $T_{s}$ & $0.002 \mu \mathrm{sec}$ \\
\hline \multicolumn{3}{|c|}{ Phase History } \\
\hline Number of points for FFT & $N_{\text {FFT }}$ & 49152 \\
\hline Spatial frequency step size & $\mathrm{d} k$ & $0.029 \mathrm{rad} / \mathrm{m}$ \\
\hline Minimum spatial frequency & $k_{\min }$ & $211.79 \mathrm{rad} / \mathrm{m}$ \\
\hline Maximum spatial frequency & $k_{\max }$ & $28.21 \mathrm{rad} / \mathrm{m}$ \\
\hline
\end{tabular}



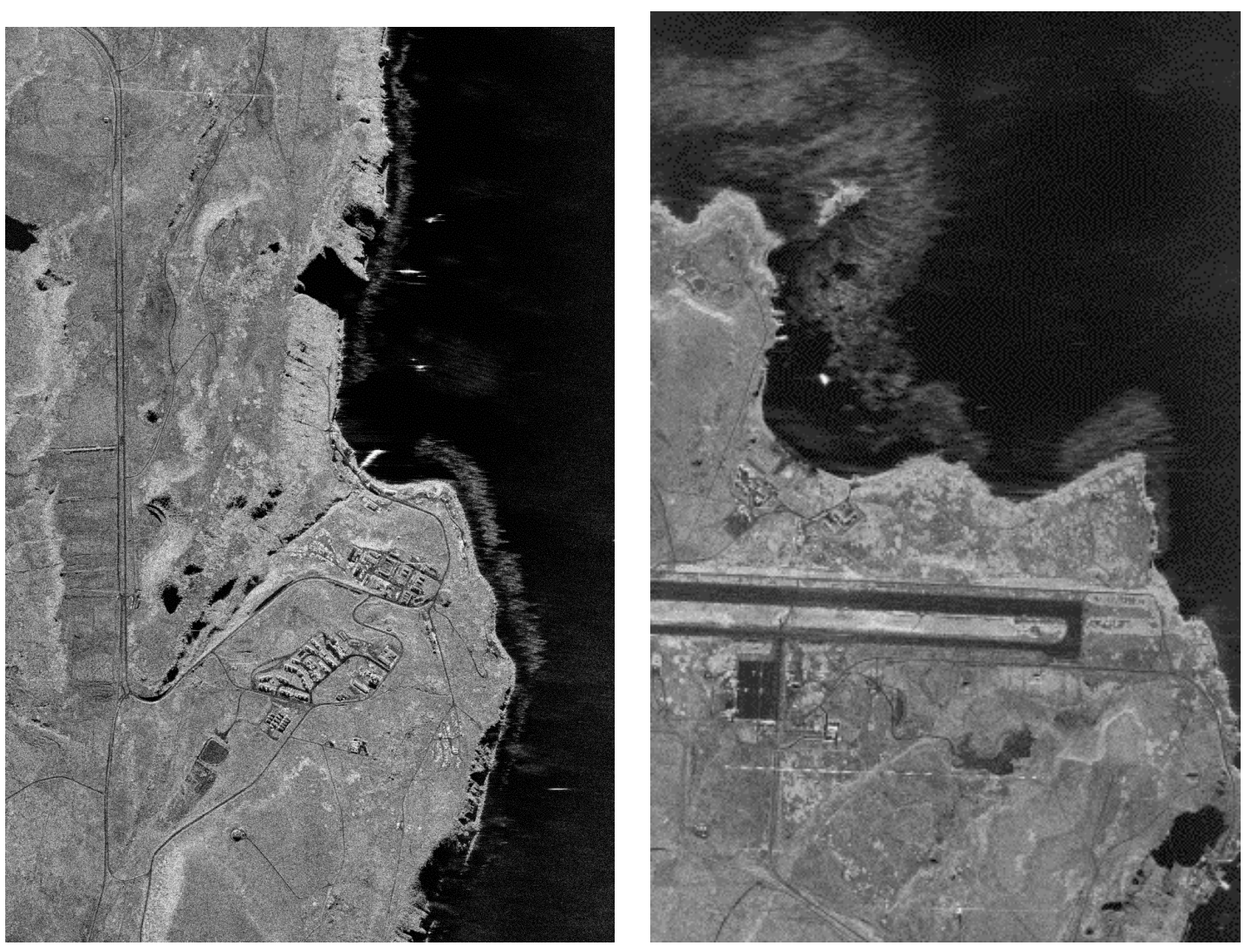

Figure 3.9: LiMIT images of the northern end of San Clemente Island. The imaged area is roughly 4 kilometers by 2 kilometers. The ocean appears dark because water absorbs electromagnetic energy. 


\subsection{Information Theory}

The purpose of remote sensing, such as SAR imaging, is to acquire information. Information theory is a set of principles for quantifying information and for evaluating the transmission of information. In this research, the similarity between a simulation image and a SAR image is measured by their mutual information; this measure and related measures are described below.

Information is defined as a reduction in uncertainty, where uncertainty is measured by entropy. Entropy is a function of the probability distribution that describes a random variable,

$$
\mathcal{H}(\mathrm{v})=-\sum_{v \in \mathcal{S}} \mathcal{P}(v) \log _{2} \mathcal{P}(v),
$$

where $\mathbf{v}$ is a random variable, $\mathcal{P}(v)$ is the probability distribution of $\mathbf{v}$ and $\mathcal{S}$ is the discrete set of values that make up the support of $v$. For example, the pixel values $|f(\tilde{x}, \tilde{y})|$ of a SAR image are realizations of a random variable and the range of values contained in the image is the support. The choice of 2 as the basis of the logarithm is historically motivated by measuring information in terms of bits, where a bit is a binary-valued unit. This unit is not meaningful in all contexts where information theory is applied; here the measure is considered unitless.

The entropy is maximum for a uniform distribution, where each value in the domain has equal probability. In other words, the uncertainty is maximum when each possible value is equally probable. The entropy of a uniform distribution for a set of $N$ possible values is

$$
\mathcal{H}(\mathrm{v})=-\sum_{i=1}^{N} \frac{1}{N} \log _{2} \frac{1}{N}=\log _{2} N .
$$


The greater the number of possible values $N$, the greater the uncertainty.

Uncertainty about the value of a random variable can be reduced by knowledge of another random variable. Conditional entropy is the entropy of one random variable given the value of another random variable,

$$
\mathcal{H}\left(\mathrm{v}_{1} \mid \mathrm{v}_{2}\right)=-\sum_{v_{1} \in \mathcal{S}_{1}} \sum_{v \in \mathcal{S}_{2}} \mathcal{P}\left(\mathrm{v}_{1} \mid \mathrm{v}_{2}\right) \log _{2} \mathcal{P}\left(\mathrm{v}_{1} \mid \mathrm{v}_{2}\right)
$$

If the uncertainty is reduced, then $v_{1}$ provides information about $v_{2}$. The amount of uncertainty reduction is the mutual information,

$$
\mathcal{I}\left(\mathrm{v}_{1}, \mathrm{v}_{2}\right)=\mathcal{H}\left(\mathrm{v}_{1}\right)-\mathcal{H}\left(\mathrm{v}_{1} \mid \mathrm{v}_{2}\right) .
$$

The mutual information between two random variables can also be calculated using joint entropy. The joint entropy of two random variables is

$$
\mathcal{H}\left(\mathrm{v}_{1}, \mathrm{v}_{2}\right)=-\sum_{v_{1} \in \mathcal{S}_{1}} \sum_{v_{2} \in \mathcal{S}_{2}} \mathcal{P}\left(v_{1}, v_{2}\right) \log _{2} \mathcal{P}\left(v_{1}, v_{2}\right)
$$

If the joint entropy is less than the sum of the individual entropies, then the two variables provide information about each other; the mutual information is

$$
\mathcal{I}\left(\mathrm{v}_{1}, \mathrm{v}_{2}\right)=\mathcal{H}\left(\mathrm{v}_{1}\right)+\mathcal{H}\left(\mathrm{v}_{2}\right)-\mathcal{H}\left(\mathrm{v}_{1}, \mathrm{v}_{2}\right) .
$$

The two expressions for mutual information in (3.33) and (3.35) are mathematically equivalent. Mutual information is also mathematically equivalent to the Kullback Leibler distance between the joint distribution of the two variables $\mathcal{P}\left(\mathrm{v}_{1}, \mathrm{v}_{2}\right)$ and the independent distribution $\mathcal{P}\left(\mathrm{v}_{1}\right) \mathcal{P}\left(\mathrm{v}_{2}\right)$ [70]. So unlike correlation, which only measures 


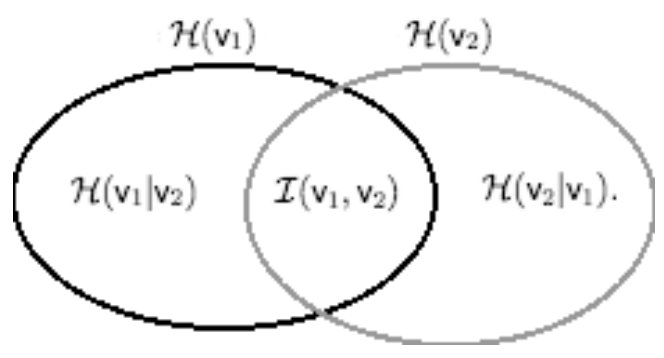

Figure 3.10: Mutual information is often illustrated by a Venn diagram. Each oval represents the entropy of a random variable; the overlapping region represents the mutual information between them.

linear dependence, mutual information is zero if and only if the two variables are statistically independent. This distinction is important for measuring the similarity of two images produced using different sensors because the pixel values of the two images may not have a linear relationship even when they are images of the same object. This same condition may occur when one image is produced using simulation.

The diagram in Figure 3.10 illustrates the following relationships and properties of mutual information, which are derived from (3.30), (3.32), (3.34), and (3.35):

$$
\begin{aligned}
& \mathcal{I}\left(\mathrm{v}_{1}, \mathrm{v}_{2}\right) \geq 0 \\
& \mathcal{I}\left(\mathrm{v}_{1}, \mathrm{v}_{2}\right) \leq \min \left(\mathcal{H}\left(\mathrm{v}_{1}\right), \mathcal{H}\left(\mathrm{v}_{2}\right)\right) \\
& \mathcal{I}\left(\mathrm{v}_{1}, \mathrm{v}_{2}\right)=\mathcal{I}\left(\mathrm{v}_{2}, \mathrm{v}_{1}\right)
\end{aligned}
$$

The lower bound (3.36) is achieved only when the two variables are independent, as noted above. The upper bound (3.37) depends on the arguments; in the development of the information extraction method in Chapter 6 a normalization is introduced so that the mutual information between different pairs of variables can be compared. The property of 3.38 is necessary for mutual information to be used as a similarity measure. 


\section{Chapter 4}

\section{Building Features in SAR Imagery}

This chapter presents a characterization of the electromagnetic response of a building in the context of SAR imaging. The total scattered field of a building is the superposition of the scattering from the roof, the vertical walls and the wall-ground dihedral (double-bounce scattering). The scattering from these components appear as features in the SAR image of a building and these features contain information about the building. The intensities of these features under different conditions were compared using electromagnetic scattering models. The results predict which component - roof, walls, or double-bounce - is expected to be the brightest feature in a SAR image for a given building orientation and radar grazing angle. The mapping of the scattered fields from all the components into a two-dimensional image is determined by the geometry of the SAR data collection process. The mapping was used here to develop new equations that relate the height, width and roof slope of a building to the SAR image features produced by the building. These two characteristics, the scattering intensity and the mapping to an image, will first be discussed separately and then combined to interpret building features in the LiMIT data. 


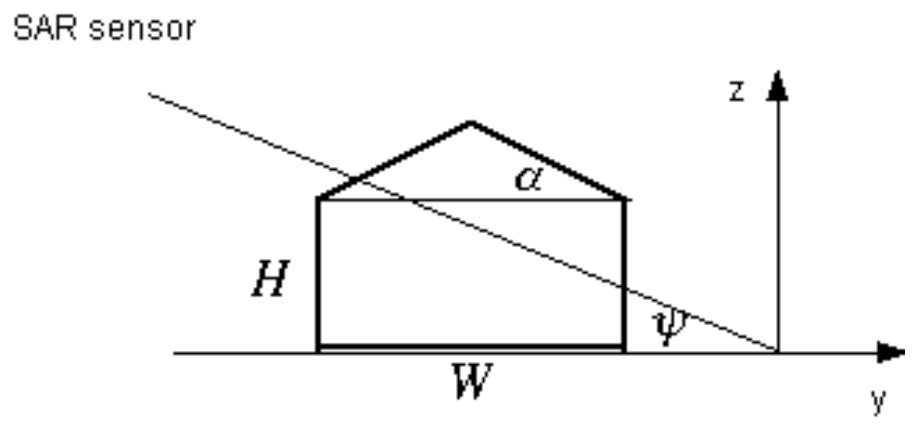

a) Side view

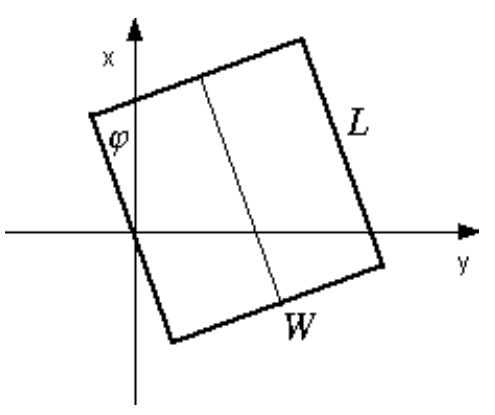

b) Top view

\begin{aligned} & \hline$H$ wall height \\ &$W$ building width \\ &$L$ building length \\ &$\alpha$ roof angle, $\alpha=0$ is flat roof \\ &$\phi$ building orientation, $\phi=0$ is broadside \\ &$\psi$ grazing angle of radar \\ & \hline\end{aligned}

Figure 4.1: Building model symbols.

\subsection{Characterization of Building Scattering}

A building is modeled as having a rectangular footprint, vertical walls and a symmetrical sloped roof (a flat roof has a slope of zero), parameterized as shown in Figure 4.1. The ground on which the building sits is assumed to be level with a rough surface. The roof and the walls are considered smooth, rectangular surfaces. The roof angle $\alpha$ of a building can vary from 0 degrees (flat) to very steep (greater than 50 degrees). Roof slope in the United States is measured by the amount of rise per foot of horizontal length. Common roof slopes used in construction are given in Table 4.1.

The building scene is defined in terms of the SAR geometry illustrated in Figure 3.4. The $z$-axis is normal to the ground and the $y$-axis is the ground projection of the SAR sensor's pointing vector, with the positive $y$ direction moving away from the sensor. This geometry is convenient for calculating the scattered fields from the 
Table 4.1: Common Roof Slopes in the United States

\begin{tabular}{rrc}
$\begin{array}{r}\text { Rise per foot } \\
\text { (in) }\end{array}$ & $\begin{array}{r}\text { Angle } \\
(\mathrm{deg})\end{array}$ & Class \\
\hline 0 & 0.0 & \\
1.5 & 7.1 & low \\
3.0 & 14.2 & \\
\hline 5.0 & 22.7 & standard \\
6.0 & 26.5 & \\
8.0 & 33.7 & \\
12.0 & 45.0 & steep \\
15.0 & 56.3 &
\end{tabular}

building because it is straightforward to construct the local scattering geometry of each building component like that of Figure 3.2. At most, two walls (and dihedrals) will be illuminated by the radar.

\subsubsection{Intensity Of Scattering From Building Components}

The purpose of modeling the scattered fields from a building is to understand which scattering components will be dominant, or even visible, in a SAR image for different building orientations and sensor configurations. This understanding aids image interpretation in terms of a building's physical attributes.

The PO model for the monostatic scattering from a rectangular surface (3.3) was used here to calculate the scattered fields from the roof and the walls. The doublebounce scattering from the wall-ground dihedral was calculated using the model of Franceschetti [25]. The model follows the procedure outlined in Section 3.1, using Geometric Optics (GO) to calculate the first scattered field and PO for the second scattered field. The rough surface of the ground is accounted for using the surface height statistics. The calculated intensity of the scattered fields are reported in terms 
of RCS using (3.9).

An alternative to the PO method for calculating the intensity of the scattered field is the Lambertian model,

$$
\mathbf{E}_{s}=\frac{k^{2}}{4 \pi} \mathrm{RCS}_{0} \cos ^{2} \theta_{i}
$$

where $\mathrm{RCS}_{0}$ is a constant, sometimes chosen to be an estimate of the scattering intensity for $\theta_{i}=0$ based on electromagnetic principles but more often arbitrary. This simple model captures the salient relationship between scattering intensity and the inicident angle. Both models have been used in the literature, but their predictions for the intensity of the scattered field are different as shown in Figure 4.2. With both models, the maximum intensity is observed when the incident wave is orthogonal to the surface. But the shape of the intensity function predicted by the two models is different. The purpose of this comparison is to demonstrate that the following evaluation of the scattered fields from a building is model-dependent.

Note that the PO model intensity function shown in Figure 4.2 has a lobing pattern which is undesirable because the fine structure of the lobing pattern exceeds a reasonable level of precision in the model parameters. To address this issue, the envelope of the PO model was used for the remainder of this work.

The scattered field (3.3) is calculated using properties of the incident field and properties of the scattering surface. The incident field, in this case, is the transmitted radar pulse. Its wavenumber is given by the center frequency of the pulse, and its propagation vector is

$$
\hat{\boldsymbol{k}}=0 \hat{\boldsymbol{x}}+\cos \psi \hat{\boldsymbol{y}}-\sin \psi \hat{\boldsymbol{z}}
$$

where $\psi$ is the grazing angle of the radar. The LiMIT sensor values, $9.72 \mathrm{GHz}$ 


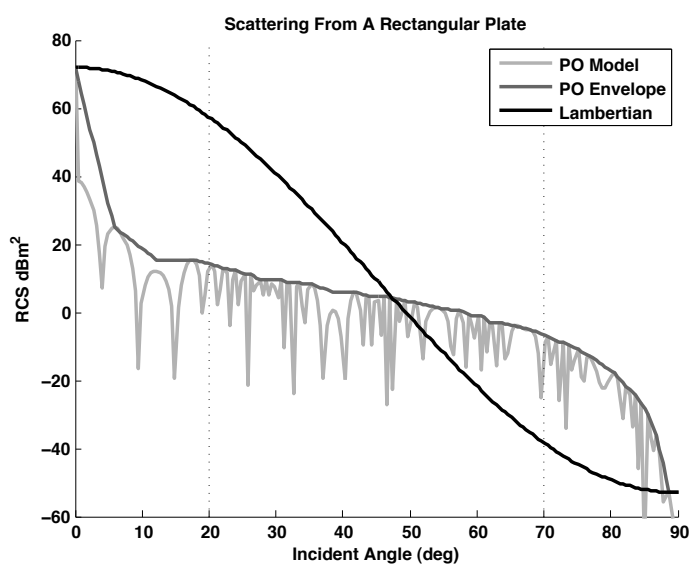

Figure 4.2: The PO model and the Lambertian model give different results. The frequency of the incident wave is $9.72 \mathrm{GHz}$, the dimensions of the surface are $10 \mathrm{~m} \times 10 \mathrm{~m}$, and its relative permittiviy is $4+\mathrm{j} 0.1$.

center frequency and $\psi=23.74$ degrees, were used for here so that the results are representative of the LiMIT imagery.

The relevant properties of the scattering surface are its dimensions, its normal vector $\hat{\boldsymbol{n}}$ and its relative permittivity $\epsilon_{r}$. The dimensions of the wall are the building height $H$ and length $L$. The dimensions of the roof are the building length $L$ and width $W$. These dimensions were all set to 10 meters to investigate the effects of the other properties. The normal vector for a vertical wall, based on the geometry of Figure 4.1, is

$$
\hat{\boldsymbol{n}}_{w}=\sin \phi \hat{\boldsymbol{x}}-\cos \phi \hat{\boldsymbol{y}}+0 \hat{\boldsymbol{z}}
$$

The normal vector for a roof with angle $\alpha$ is

$$
\hat{\boldsymbol{n}}_{r}=\sin \phi \sin \alpha \hat{\boldsymbol{x}}-\cos \phi \sin \alpha \hat{\boldsymbol{y}}+\cos \alpha \hat{\boldsymbol{z}}
$$

The intensity of the scattered field is highly dependent on the incident angle of the 


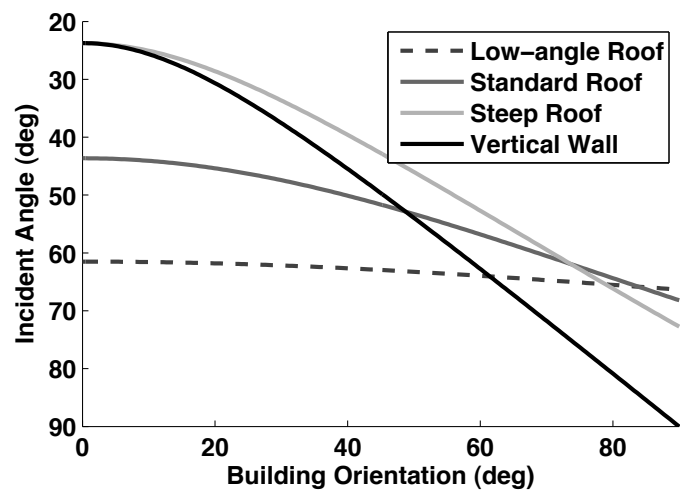

Figure 4.3: Building orientation influences the incident angle of the radar pulse on the surfaces of a building. The incident angle was calculated using the grazing angle of the LiMIT sensor, approximately 24 degrees.

transmitted pulse on the surface. The incident angle is calculated from the propagation vector (4.2) and the surface normal,

$$
\theta_{i}=\cos ^{-1}(-\hat{\boldsymbol{k}} \cdot \hat{\boldsymbol{n}})
$$

The incident angle on a wall and a sloped roof as a function of building orientation $\phi$ is shown in Figure 4.3, using the LiMIT grazing angle. The incident angle changes at different rates for the wall and each of the differently sloped roofs, indicating that the relative scattering intensities of the wall and the roof will change with building orientation, and that the slope of the roof is also an influence.

The RCS of a sloped roof as a function of building orientation is plotted in Figure 4.4 - The steeper the slope of the roof, the greater the effect of the building's orientation on the RCS. In other words, the scattering intensity from a steeply sloped roof will decrease more rapidly than that of a flat or low sloped roof as the building's orientation moves away from broadside.

The PO scattered fields from the wall and roof depend on dielectric permittivity, 


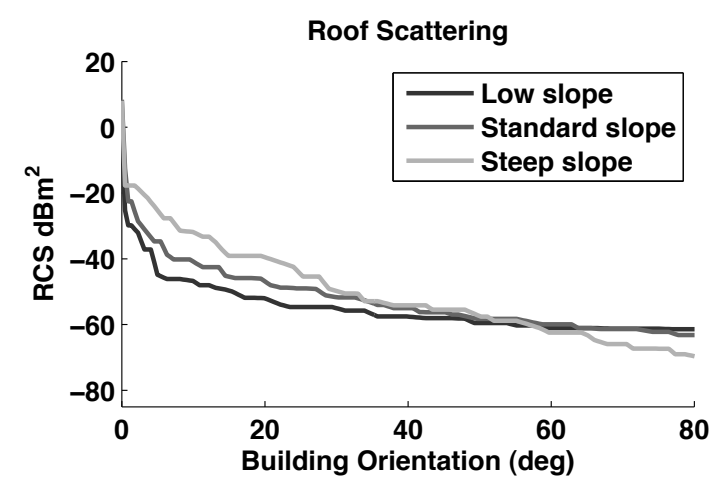

Figure 4.4: The RCS of a sloped roof at the LiMIT grazing angle of 23.74 degrees and frequency of $9.72 \mathrm{GHz}$.

Table 4.2: Dielectric Constants and Roughness

\begin{tabular}{crccc} 
Material & $\varepsilon_{r}$ & $\sigma$ & $\ell_{c}$ & Reference \\
\hline wall, roof & $4+\mathrm{j} 0.1$ & smooth & {$[25,22]$} \\
wall, roof (dry concrete) & $6+\mathrm{j} 0.5$ & \multicolumn{2}{c}{ smooth } & {$[23]$} \\
wall (brick) & $4.5+\mathrm{j} 0.3$ & \multicolumn{2}{c}{ smooth } & {$[31]$} \\
ground (soil) & $4+\mathrm{j} 0.1$ & 0.08 & 0.3 & {$[25]$} \\
ground & $4+\mathrm{j} 0.1$ & 0.003 & 0.05 & {$[25]$} \\
ground (wet soil) & $12+\mathrm{j} 3.0$ & \multicolumn{2}{c}{ not given } & {$[23]$} \\
ground (asphalt) & $7+\mathrm{j} 0.3$ & 0.0025 & 0.012 & {$[28]$} \\
ground (grass) & $9+\mathrm{j} 2.0$ & 0.010 & 0.057 & {$[28]$}
\end{tabular}

which is usually not known. Likewise the GO-PO model for the double bounce depends on the permittivity and also depends on the roughness statistics of the ground surface. The statistics used are the standard deviation $\sigma$ of the surface height from the mean plane, and the correlation length $\ell_{c}$ of the surface height. The dielectric constants and roughness parameters published in the literature are summarized in Table 4.2 .

The effect of the permittivity value on the predicted RCS is shown in Figure 4.5. The difference between the RCS curves using three different permittivity values from the literature is not as significant as the change in RCS due to the building orientation. 


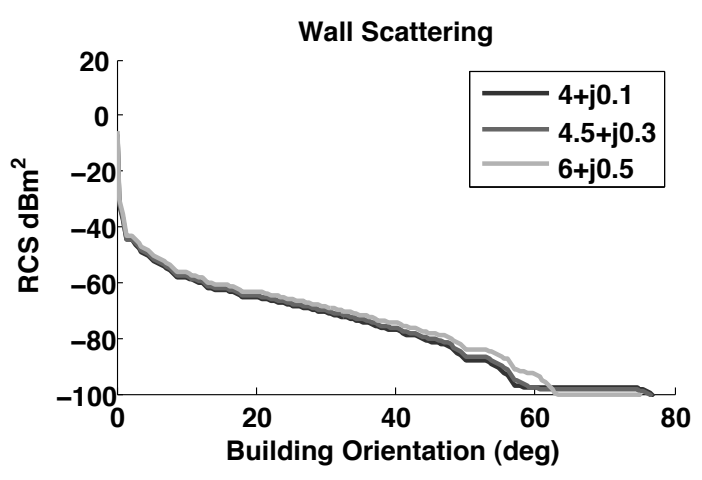

a.

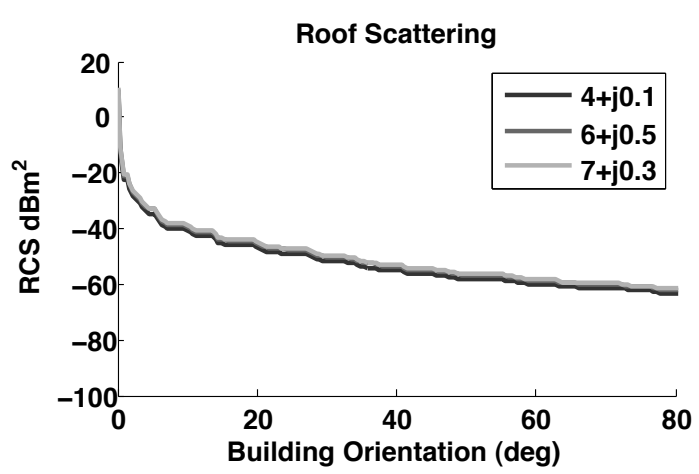

b.

Figure 4.5: The RCS of a vertical wall (a) and a standard sloped roof (b) at the LiMIT grazing angle of 23.74 degrees and frequency of $9.72 \mathrm{GHz}$ for different permittivity values.

This indicates that the specific choice of permittivity is not critical for modeling a building's electromagnetic response using the PO model.

The effect of permittivity and surface roughness on the intensity of the double-bounce scattering is shown Figure 4.6. The roughness has a significant effect on the doublebounce scattering. For a building surrounded by asphalt, the double-bounce scattering is insensitive to the building orientation and will be the brightest image feature for any building orientation. For a building surrounded by grass, the double-bounce scattering will be dominant only for building orientations from 0 (broadside) to about 10 degrees, at which point the scattering becomes insignificant relative to the wall and roof scattering.

Putting it all together, the RCS of all the building components are plotted together in Figure 4.7. This plot is could be used to interpret the building features in a SAR image. For example, if the building orientation were less than 30 degrees, than bright features would be due to double-bounce scattering. If the roof had a standard slope, then it would produce a brighter feature than the walls would. These predictions 


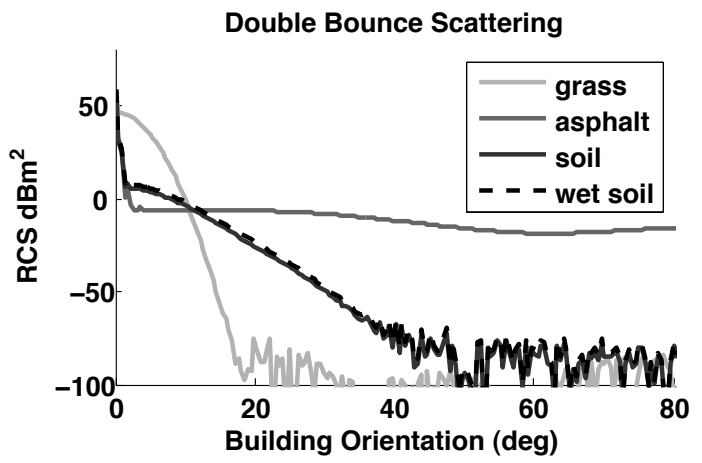

Figure 4.6: The RCS of the double bounce scattering from the wall-ground dihedral at the LiMIT grazing angle of 23.74 degrees and frequency of $9.72 \mathrm{GHz}$. The roughness of the surface changes the response of the double-bounce scattering to the building orientation angle.

were used to interpret the building features observed in the LiMIT imagery described in Section 4.2.3.

\subsubsection{Scattering Components Mapped To Image Features}

Comparing the RCS values of the building scattering components predicted by the PO model does not tell the whole story. The geometry of the collection and the shape of the building influence the appearance of the three-dimensional building in the two-dimensional image. In the image, the scattering from the vertical wall is superimposed with the scattering of the ground, due to the layover effect. Layover refers to the way points at some height above the ground plane appear closer in range in the image. For example, the top of the wall and the base of the wall are both at the same range location in the three-dimensional scene, but the top of the wall will appear closer in the image. The scattering from the roof and the wall may overlap in the image, as well. Figure 4.8 illustrates how the shape of a building influences the way in the which the scattering components are combined in the image. 


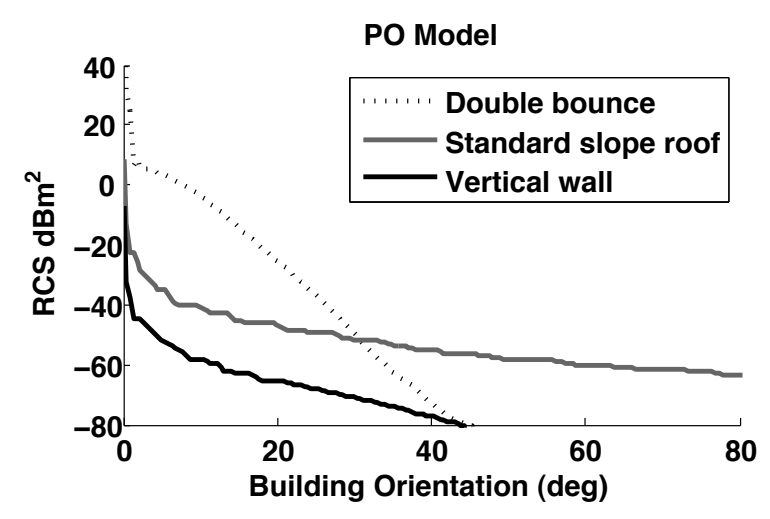

Figure 4.7: The PO model is useful for predicting which scattering component will be dominant for a given building orientation in the scene. The wall and roof have relative permittivity $\epsilon_{r}=4+\mathrm{j} 0.01$, the double-bounce is for ground composed of soil.

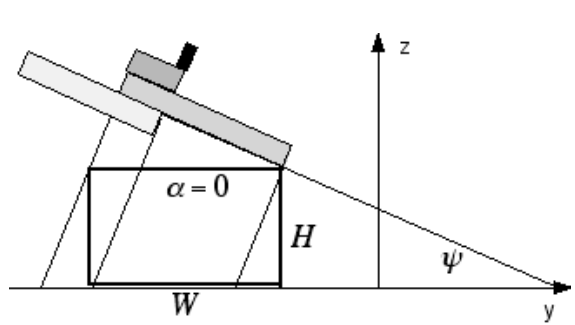

a) flat roof

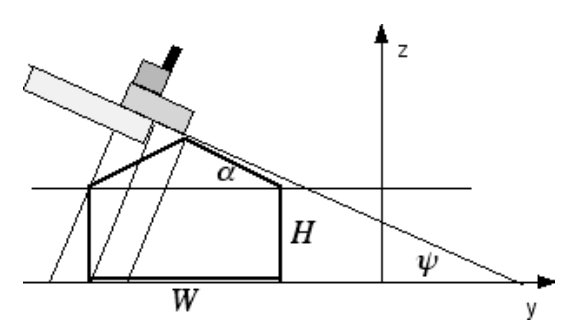

c) roof slope $>$ grazing angle

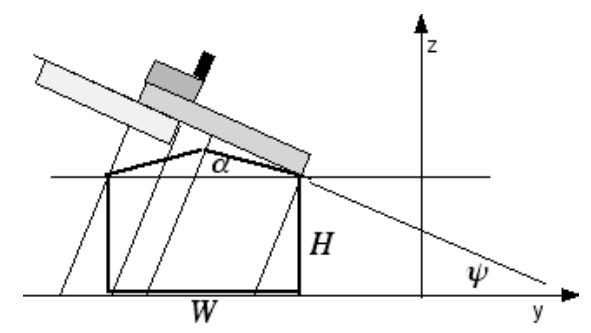

b) roof slope $<$ grazing angle

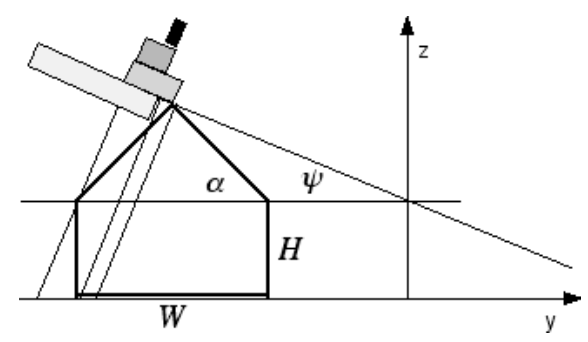

d) roof slope $\gg$ grazing angle

Figure 4.8: Each of the figures shows a building with vertical height $H$ and width $W$ illuminated at a grazing angle of $\psi$. The plot above each building shows the width and relative location of the scattering components in the slant plane image. The light gray strip is the ground scattering, the medium gray strip is the roof scattering, the dark gray strip is the wall scattering and the black strip is the double-bounce scattering. 
In order to quantify the effects of the building parameters on the image appearance, two features of the building SAR signature were identified: the width of the scattering from the roof $\tilde{W}$, and the width of the scattering from the wall $\tilde{H}$. The tilde denotes that these are image features, and not the physical building parameters. An analysis of the mapping from scene coordinates to image coordinates was performed to relate the building parameters to the image features for a given SAR collection. The analysis is based on the geometry of Jakowatz [71], which does not make any assumptions about the sensor trajectory except that it is a straight line. This general framework is necessary to include the effects of a squinted geometry on the mapping.

The projection of a point $[x, y, z]$ in ground coordinates to a point $[\tilde{x}, \tilde{y}]$ in slant plane image coordinates is

$$
\begin{aligned}
\tilde{y} & =y \cos \psi-z \sin \psi, \\
\tilde{x} & =x \cos \varphi-y \sin \psi \sin \varphi+z \cos \psi \sin \varphi,
\end{aligned}
$$

where $\psi$ is the grazing angle and $\varphi$ is the tilt angle [71]. For a broadside collection, i.e., not squinted, the tilt angle is zero. The mapping given by (4.6) and (4.7) was used to quantify the width of the roof reflection in the image, the width of the wall reflection, and the amount of overlap of these reflections in terms of the building parameters $H, W$ and $\alpha$.

Consider the points labeled in Figure 4.9. All points can be defined in ground coordinates given $P_{1}$ and the building parameters. The definition of each of the points is given in Table 4.3. The mapping of these points to image coordinates is given by (4.7) and (4.6). 

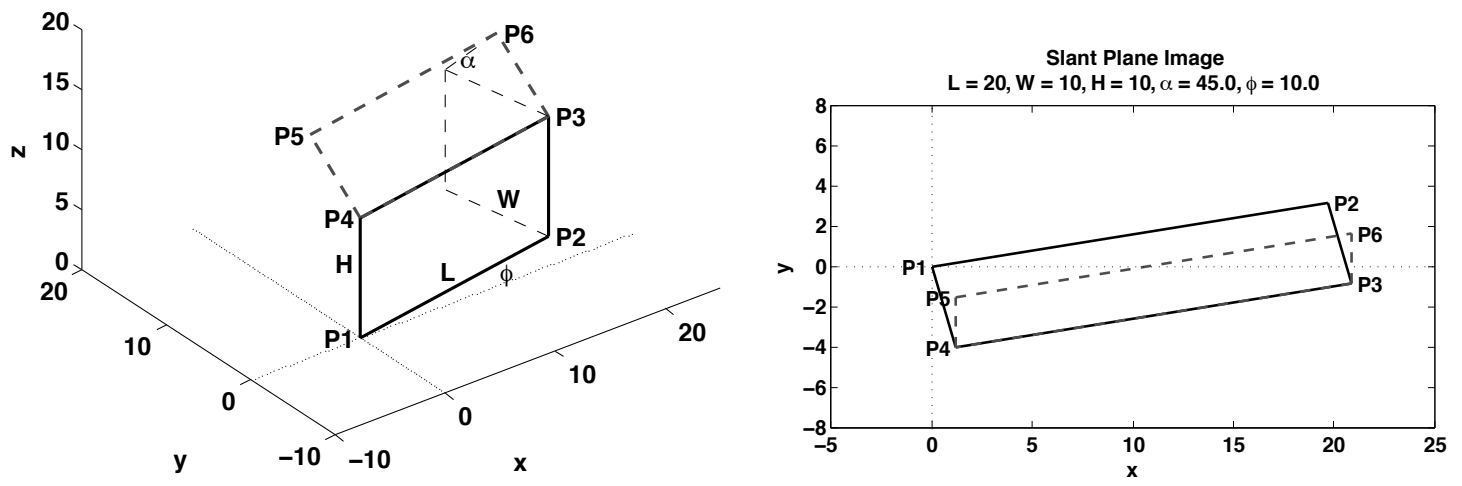

Figure 4.9: Building points for geometric model. The points in the three-dimensional scene (left) are mapped to corresponding points in the slant plane image (right).

Table 4.3: Building Points in Ground Coordinates

\begin{tabular}{c|lll} 
Building & & & \\
Point & $x$ & $y$ & $z$ \\
\hline$P_{1}$ & $x_{1}$ & $y_{1}$ & 0 \\
$P_{2}$ & $x_{1}+L \cos \phi$ & $y_{1}+L \sin \phi$ & 0 \\
$P_{3}$ & $x_{1}+L \cos \phi$ & $y_{1}+L \sin \phi$ & $H$ \\
$P_{4}$ & $x_{1}$ & $y_{1}$ & $H$ \\
$P_{5}$ & $x_{1}-(W / 2) \sin \phi$ & $y_{1}+(W / 2) \cos \phi$ & $H+(W / 2) \tan \alpha$ \\
$P_{6}$ & $x_{1}+L \cos \phi-(W / 2) \sin \phi$ & $y_{1}+L \sin \phi+(W / 2) \cos \phi$ & $H+(W / 2) \tan \alpha$
\end{tabular}


The mapped points were used to examine the appearance of the building in the image. An example mapping is shown in Figure 4.9. The rectangles of the roof and the wall are distorted and superimposed. The rectangles become parallelograms with corners that are not right angles. The top of the wall, $\tilde{P}_{4}$, always appears closer in range than the base of the wall $\tilde{P}_{1}$. The double bounce reflection appears at the base of the wall between $\tilde{P}_{1}$ and $\tilde{P}_{2}$. Using the mapped points, the building signature features $\tilde{H}$ and $\tilde{W}$ were quantified in terms of the building parameters and the collection geometry.

The angle between the front edge of the building with the $\tilde{x}$-axis in the image is found using $\tilde{P}_{1}$ and $\tilde{P}_{2}$ or $\tilde{P}_{4}$ and $\tilde{P}_{3}$. The slope of the line through $\tilde{P}_{4}$ and $\tilde{P}_{3}$ relative to the image cross-range dimension is

$$
\begin{aligned}
\tan \tilde{\phi}_{1} & =\frac{\tilde{y}_{3}-\tilde{y}_{4}}{\tilde{x}_{3}-\tilde{x}_{4}} \\
& =\frac{\sin \phi \cos \psi}{\cos \phi \cos \varphi-\sin \phi \sin \psi \sin \varphi}
\end{aligned}
$$

which shows that the aspect angle of the building in the image is not the same as the aspect angle in the physical scene. If the collection is not squinted, then the tilt angle is zero and (4.8) reduces to the tan of the aspect angle in the physical scene scaled by the cos of the grazing angle. A squinted geometry introduces an additional non-linear effect. The effect is small for the LiMIT grazing angle of 23.7 deg. and a squint angle of $17.92 \mathrm{deg}$ (Figure 4.10); the difference between an actual aspect angle of $45 \mathrm{deg}$. and the angle in the image is 3.75 deg., compared to $2.53 \mathrm{deg}$. for a non-squinted geometry. But the use of the image aspect angle provides a more accurate calculation of the image feature $\tilde{W}$ and $\tilde{H}$ when the aspect angle is greater than 10 degrees.

The angle between the wall feature in the image and the $\tilde{y}$-axis is found using $\tilde{P}_{4}$ and 


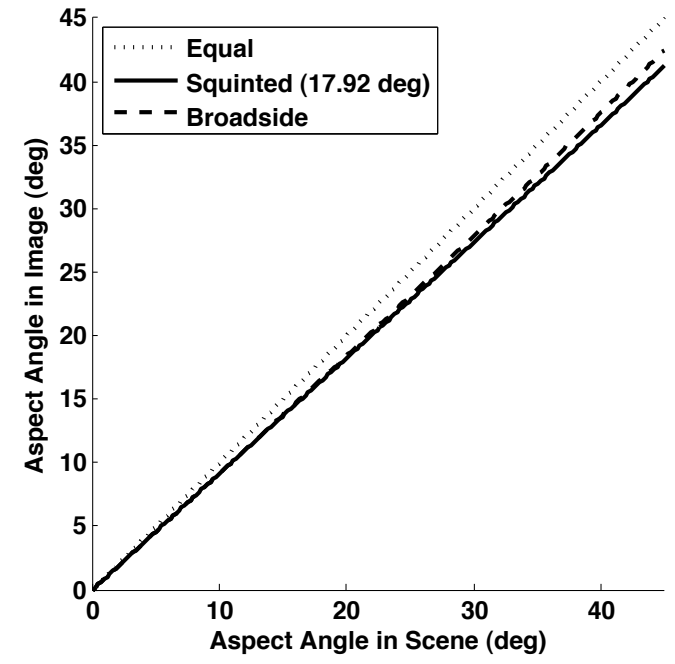

Figure 4.10: The difference between the actual aspect angle and the angle in the image increases with the aspect angle, but remains less than 4 deg.

$\tilde{P}_{1}$

$$
\begin{aligned}
\tan \tilde{\phi}_{2} & =\frac{\tilde{x}_{4}-\tilde{x}_{1}}{\tilde{y}_{4}-\tilde{y}_{1}} \\
& =\frac{\cos \psi \sin \varphi}{\sin \psi} \\
& =\frac{\sin \varphi}{\tan \psi},
\end{aligned}
$$

which is independent of the aspect angle and provides no information about the building parameters.

The angle between the roof feature in the image and the $\tilde{y}$-axis is found using $\tilde{P}_{4}$ and 
$\tilde{P}_{5}$,

$$
\begin{aligned}
\tan \tilde{\phi}_{3} & =\frac{\tilde{x}_{5}-\tilde{x}_{4}}{\tilde{y}_{5}-\tilde{y}_{4}} \\
& =\frac{\sin \phi \cos \varphi-\cos \phi \sin \psi \sin \varphi+\tan \alpha \cos \psi \sin \varphi}{\cos \phi \cos \psi-\tan \alpha \sin \psi}
\end{aligned}
$$

which, for a squinted geometry, depends on the aspect angle and contains information about the slope of the roof.

The width of the wall reflection in the slant plane image is

$$
\begin{aligned}
\tilde{H} & =\left(\tilde{y}_{1}-\tilde{y}_{4}\right)+\left(\tilde{x}_{1}-\tilde{x}_{4}\right) \tan \tilde{\phi}_{1} \\
& =H\left[\sin \psi-\cos \psi \sin \varphi \tan \tilde{\phi}_{1}\right] .
\end{aligned}
$$

So for a broadside geometry (no squint), the image wall feature is completely determined by the grazing angle, which is known, and the height of the wall. For a squinted geometry, there is a slight dependence on the aspect angle.

The width in the image range dimension of the roof reflection was found in the same way as that of the wall reflection, using $\tilde{P}_{4}$ and $\tilde{P}_{5}$,

$$
\begin{aligned}
\tilde{W}= & \left(\tilde{y}_{5}-\tilde{y}_{4}\right)-\left(\tilde{x}_{5}-\tilde{x}_{4}\right) \tan \tilde{\phi}_{1} \\
= & (W / 2)[|\cos \phi \cos \psi-\tan \alpha \sin \psi| \\
& \left.+(\sin \phi \cos \varphi+\cos \phi \sin \psi \sin \varphi+\tan \alpha \cos \psi \sin \varphi) \tan \tilde{\phi}_{1}\right] .
\end{aligned}
$$


The effect of the building physical parameters on the image features can be quantified:

$$
\begin{aligned}
\frac{\mathrm{d} \tilde{H}}{\mathrm{~d} H}= & \sin \psi-\cos \psi \sin \varphi \tan \tilde{\phi}_{1} \\
\frac{\partial \tilde{W}}{\partial(W / 2)}= & |\cos \phi \cos \psi-\tan \alpha \sin \psi| \\
& +(\sin \phi \cos \varphi+\cos \phi \sin \psi \sin \varphi+\tan \alpha \cos \psi \sin \varphi) \tan \tilde{\phi}_{1} \\
\frac{\partial \tilde{W}}{\partial \tan \alpha}= & -(W / 2)\left(\sin \psi-\cos \psi \sin \varphi \tan \tilde{\phi}_{1}\right) .
\end{aligned}
$$

From these equations, it is clear that the grazing angle of the sensor determines the sensitivity of the image features to the physical parameters. A squinted geometry with a negative tilt angle, such as that of LiMIT, increases the sensitivity.

Let the resolution of the image with respect to the building parameters be defined as the minimum difference in the parameters that could be determined from the image. For a difference to be detectable, the change in the slant plane widths of the roof and the wall reflections must be greater than the image range resolution $\rho_{y}$. Using equations (4.13) to (4.15), the resolution of the building parameters in terms of the 

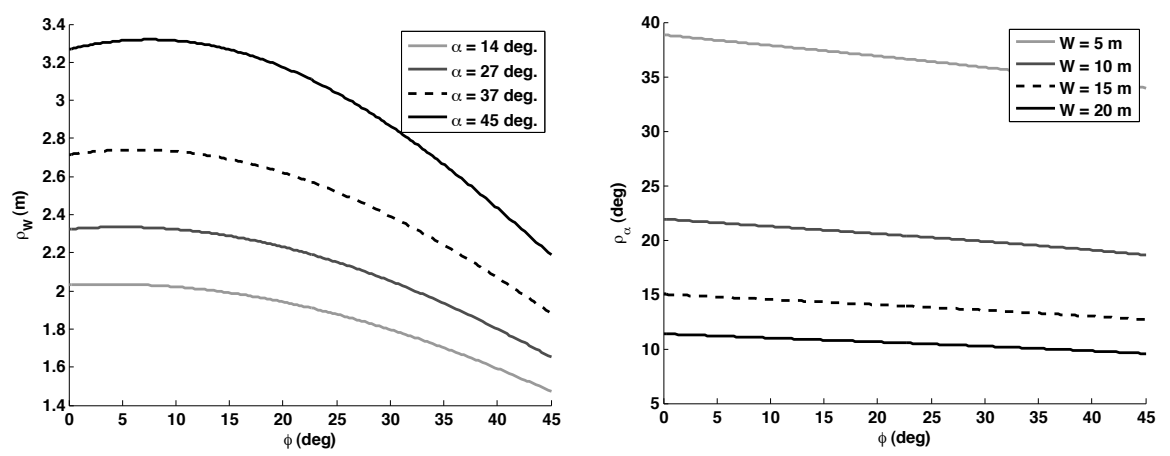

Figure 4.11: Building parameter resolution as a function of the aspect angle.

image resolution is

$$
\begin{aligned}
\rho_{H}= & \frac{\rho_{y}}{\sin \psi-\cos \psi \sin \varphi \tan \tilde{\phi}_{1}}, \\
\rho_{W}= & \rho_{y}(|\cos \phi \cos \psi-\tan \alpha \sin \psi| \\
& \left.+(\sin \phi \cos \varphi+\cos \phi \sin \psi \sin \varphi+\tan \alpha \cos \psi \sin \varphi) \tan \tilde{\phi}_{1}\right)^{-1}, \\
\rho_{\alpha}= & \frac{2 \rho_{y}}{W\left(\sin \psi-\cos \psi \sin \varphi \tan \tilde{\phi}_{1}\right)} .
\end{aligned}
$$

For LiMIT, the resolution of the building height is approximately 2 meters at an aspect angle of zero. As the aspect angle increases, the resolution improves to approximately 1.7 meters at an aspect angle of 45 degrees. However, as the aspect angle increases, the scattering intensity of the wall decreases making it more difficult to detect the wall feature in the image. The resolution of the building width $W$ depends on the roof slope, and vice-versa. The resolution for different values of these parameters is shown in Figure 4.11.

The equations for $\tilde{H}$ (4.11) and $\tilde{W}$ (4.12) show theoretical relationships between building parameters and image features that have not previously been made explicit. 
The equations (4.16) and (4.18) provide theoretical bounds on the precision of the building parameter information contained in the features. These bounds establish a reference for evaluating the performance of an information extraction method.

\subsection{Interpretation of Buildings in LiMIT Data}

The previous section provided predictions of the dominant building features for a given building orientation and quantified the location and extent of those features in a SAR image. These results are used here to interpret building features in the LiMIT SAR imagery.

\subsubsection{Buildings in LiMIT Data}

The LiMIT dataset used for this research was collected during a test mission at San Clemente Island, CA. The buildings in the dataset chosen for analysis are shown in Figure 4.12. These buildings were chosen because their images have bright features, they represent two different types of buildings and some ground truth was available for them. The L-shaped building, which will be referred to as the L-building, is a large building with two wings and a sloped roof. It was oriented at 45 degrees to the sensor so that a maximum of its surface was illuminated. The three smaller buildings, which will be referred to as the barns, have sloped roofs and are oriented broadside to the sensor with the roof ridge parallel to the sensor pointing vector. Each of these buildings was present in four different CPIs that were collected during one flight. The geometry of these CPIs and the image coordinates of the buildings are given in Table 4.4 . 


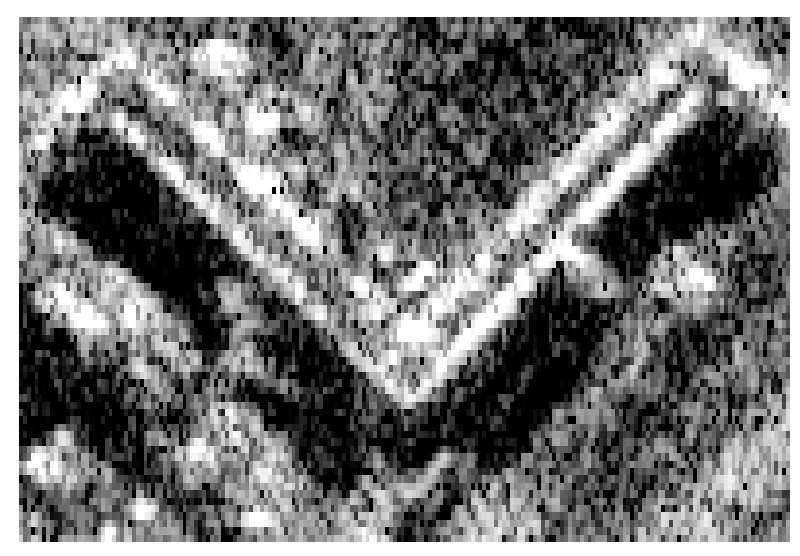

a) L-building

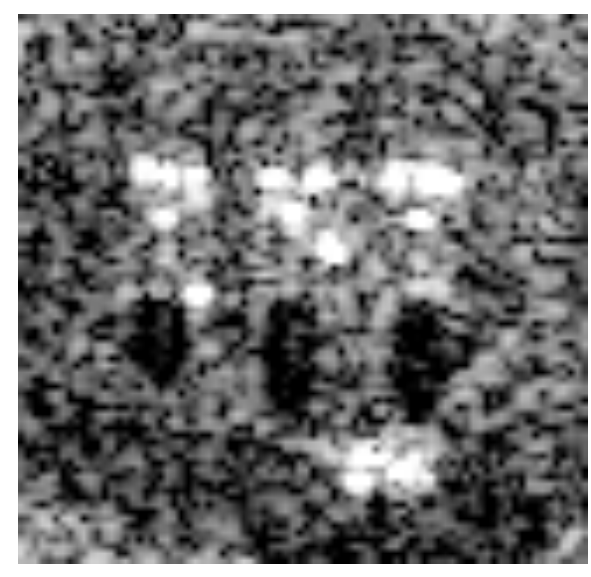

b) Barns.

Figure 4.12: The buildings used for analysis from the LiMIT dataset.

Table 4.4: LiMIT CPI Geometry

All angles in degrees.

\begin{tabular}{ccccccc}
\hline & & graze & tilt & squint & L-building & Barns \\
CPI & ID & $\psi$ & $\varphi$ & $\theta_{s}$ & $\tilde{x}, \tilde{y}$ & $\tilde{x}, \tilde{y}$ \\
\hline 1 & 6616781 & 23.74 & -7.48 & 17.92 & $-379,200$ & $-762,1498$ \\
2 & 6616782 & 23.96 & -6.68 & 15.99 & $-383,450$ & $-801,1736$ \\
3 & 6616783 & 24.16 & -5.69 & 13.62 & $-387,667$ & $-840,1940$ \\
4 & 7037707 & 24.14 & -5.67 & 13.58 & $-375,165$ & $-842,1432$
\end{tabular}


Table 4.5: L-Building Parameters

\begin{tabular}{ccc} 
Parameter & Estimate & Source \\
\hline$L_{1}, L_{2}$ & $200 \mathrm{ft}(62.5 \mathrm{~m})$ & USGS NMV \\
$W_{1}, W_{2}$ & $48 \mathrm{ft}(14.64 \mathrm{~m})$ & USGS NMV \\
$H$ & unknown & no information \\
$\alpha$ & $>0 \mathrm{deg}$. & optical image \\
$\phi$ & $45 \mathrm{deg}$. & navigation data
\end{tabular}

Table 4.6: Barn Parameters

\begin{tabular}{ccccc} 
Parameter & Barn 1 & Barn 2 & Barn 3 & Source \\
\hline$L$ & $24 \mathrm{ft}(7.32 \mathrm{~m})$ & $24 \mathrm{ft}(7.32 \mathrm{~m})$ & $24 \mathrm{ft}(7.32 \mathrm{~m})$ & USGS NMV \\
$W$ & $48 \mathrm{ft}(14.63 \mathrm{~m})$ & $48 \mathrm{ft}(14.63 \mathrm{~m})$ & $48 \mathrm{ft}(14.63 \mathrm{~m})$ & USGS NMV \\
$H$ & $12 \mathrm{ft}(3.66 \mathrm{~m})$ & $20 \mathrm{ft}(6.1 \mathrm{~m})$ & $20 \mathrm{ft}(6.1 \mathrm{~m})$ & optical image \\
$\alpha$ & $14 \mathrm{deg}$. & $14 \mathrm{deg}$. & $14 \mathrm{deg}$. & optical image \\
$\phi$ & $0 \mathrm{deg}$. & $0 \mathrm{deg}$. & $0 \mathrm{deg}$. & navigation data
\end{tabular}

The available ground truth for the buildings in the LiMIT imagery consists of optical images taken at the test site and from USGS satellite imagery. From the USGS National Map Viewer (NMV) [http://viewer.nationalmap.gov/viewer], the size of the building footprints were determined using the viewer's measurement tool. Additional information was inferred from the test site optical images and from knowledge of U.S. building conventions. In the U.S., lumber is typically sold in lengths of 8, 12 and 16 feet. A standard roof slope has 4 in. of rise per foot of horizontal length which is an angle of 22.5 deg. Standard or lower sloped roofs are typically found in climates where snowfall is rare, such as that of the test site. A summary of the known building parameters is given in Tables 4.5 and 4.6 . 


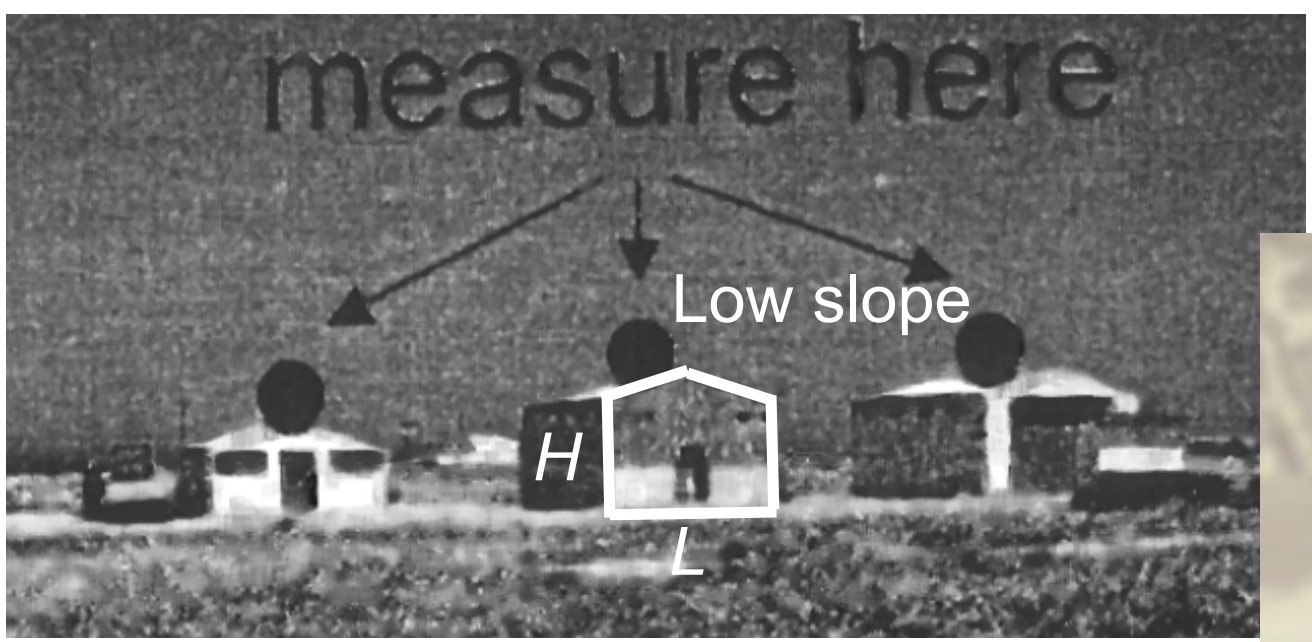

Figure 4.13: From this photograph of the three barns, it appears that the height of Barn 2 (center) is slightly less than it's length; Barn 1 (left) is shorter and Barn 3 (right) is about the same height. Note what appears to be vehicles or machinery to the left of Barn 1 and in front of Barn 3; these could be strong scatterers.

\subsubsection{Analysis of Building Image Features}

The width of the image features in the range dimension was related to the building's height and width in Section 4.1.2. The width of the building features in the LiMIT images was measured using range profiles. A range profile is a plot of pixel intensity vs. range at a particular cross-range location. For the digitized images, a range profile is a plot of one column of the image pixel matrix.

The building examples was present in four CPIs from the same flight. For each building example, the region of one CPI image containing the building was manually selected so that all the building features, including the shadow, were contained in the region and the amount of background contained in the region was small. This reference region was then registered with each of the other three CPIs using the mutual information registration method described in Chapter 6. 


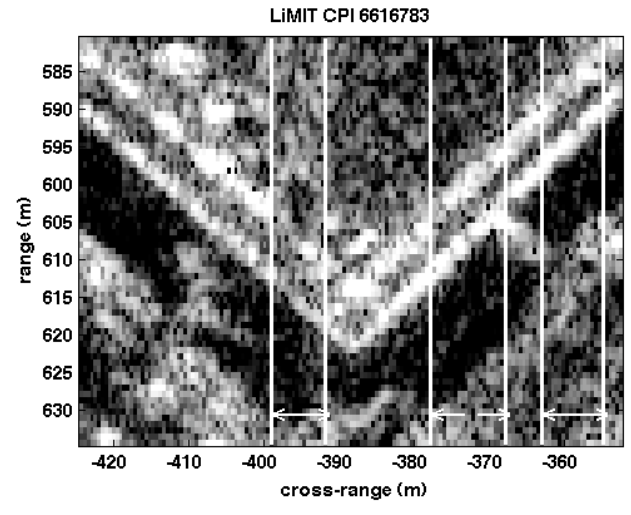

a) L-building.

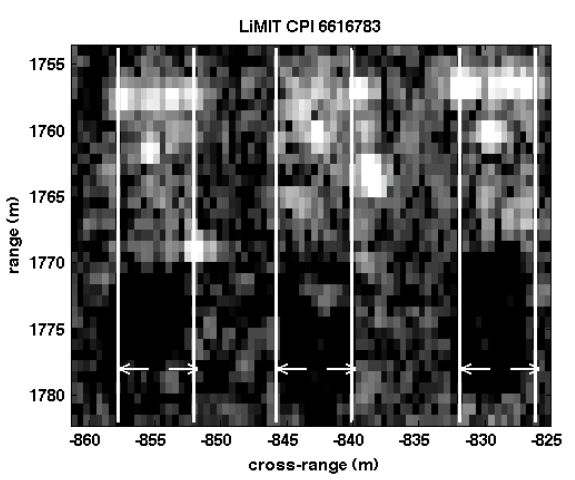

b) Barns.

Figure 4.14: The white lines show the regions used for analysis of the L-building (a) and the barns (b). Each column of image pixel intensity values within the regions was plotted as a range profile.

For the L-building, three regions were identified as being relatively free of scatterers other than the building itself. These regions are shown on one of the CPI images in Figure 4.14a. The same regions were used for each of the CPIs. For the barns, the three regions corresponding to the individual barns were identified, as shown in Figure 4.14b. All the range profiles in all of these regions were used for the analysis.

The following features were measured for each of the range profiles: the length of the total building scattering, the distance between the first and second peaks, the offset of the first peak from the start of the building scattering, and the offset of the second peak from the end of the building scattering, and the intensity of the two peaks. These features are shown on an example range profile at the top of Figure 4.15. The start of the building scattering was determined the first of at least three consecutive pixels with intensity greater than a threshold. The threshold was calculated for each CPI as the median intensity of the image plus half the interquartile range. The end of the bulding scattering (start of shadow) was determined as the last pixel before three consecutive pixels with intensity less than the median image intensity. 


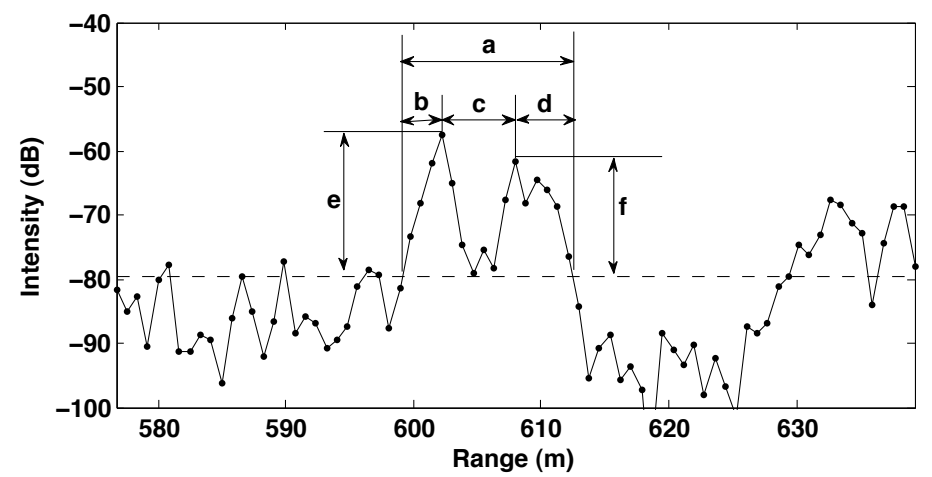

a) Building scattering width.

b) Offset of 1st peak.
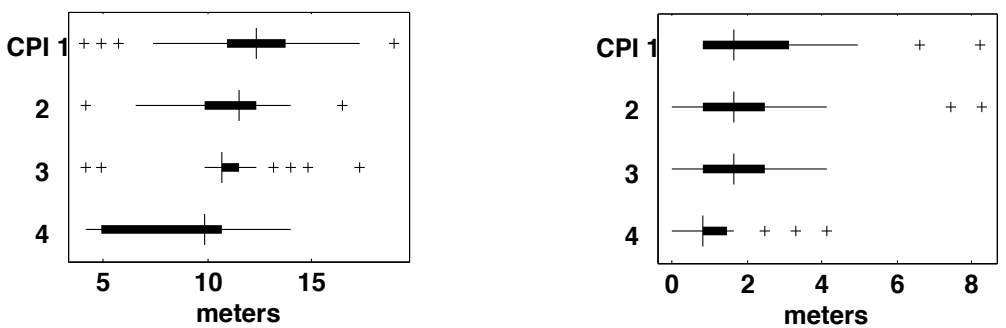

c) Distance between peaks.

d) Offset of 2nd peak.
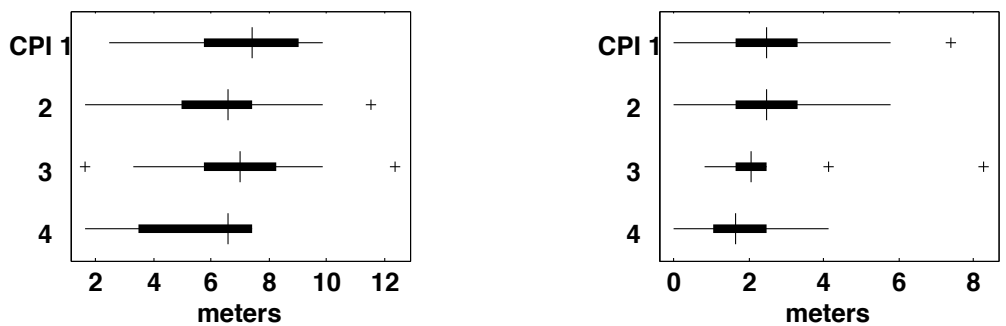

e) Intensity of 1st peak.

f) Intensity of 2 nd peak.
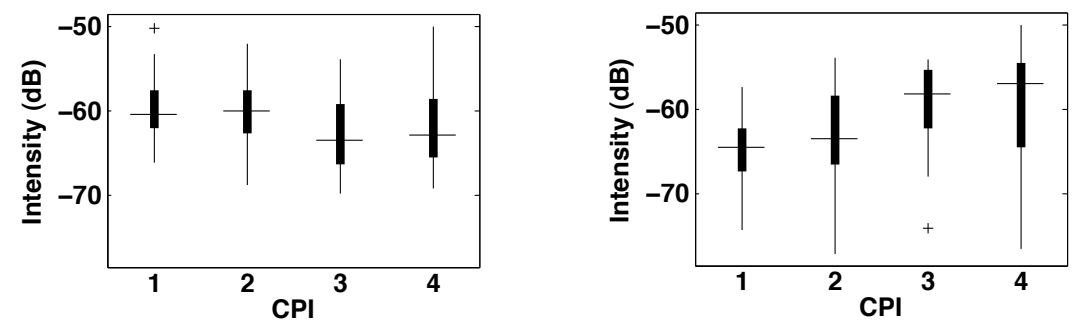

Figure 4.15: The results of the L-building range profile analysis are shown separately for each CPI. 


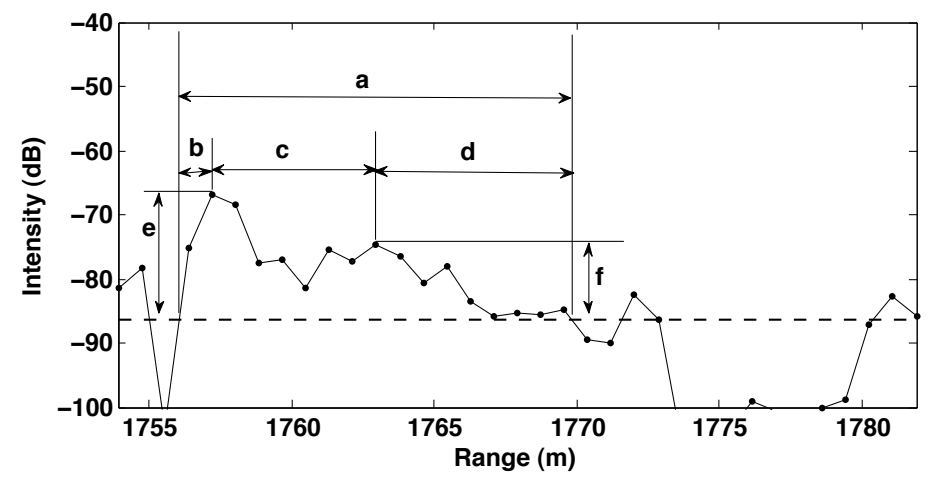

a) Building scattering width.

b) Offset of 1st peak.

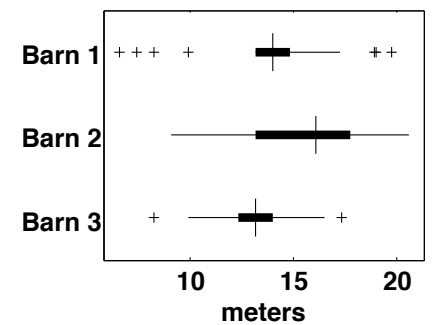

c) Distance between peaks.

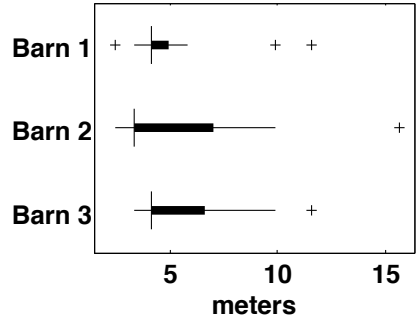

e) Intensity of 1st peak.

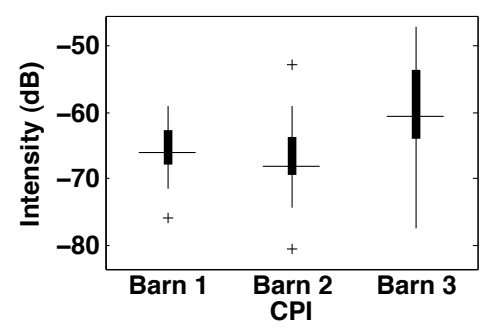

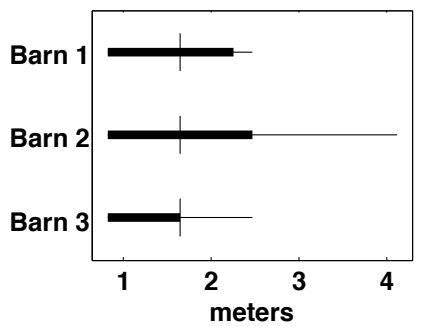

d) Offset of 2nd peak.

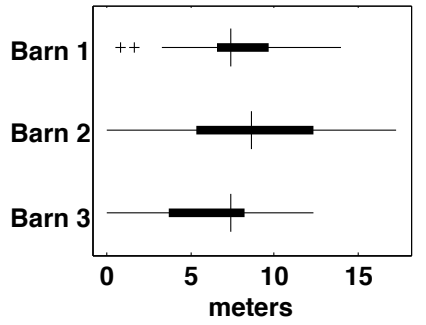

f) Intensity of 2 nd peak.

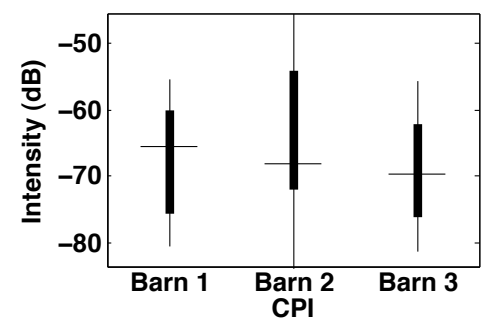

Figure 4.16: The results of the range profile analysis of the three barn-like buildings. In the peak intensity plot, the lighter bars correspond to the first peak (closest to sensor) and darker bars to the second peak. The height of each bar is the maximum value, and the two horizontal lines mark the mean and minimum. 
Table 4.7: Summary of range profile analysis.

\begin{tabular}{l|rl} 
Feature & Mean & Std \\
\hline \multicolumn{3}{l}{ L-building } \\
\hline a) Building scattering width & $10.8 \mathrm{~m}$ & $3.2 \mathrm{~m}$ (3.9 pixels) \\
b) Offset of first peak & $1.9 \mathrm{~m}$ & $1.7 \mathrm{~m}$ (2.0 pixels) \\
c) Distance between peaks & $6.5 \mathrm{~m}$ & $2.2 \mathrm{~m}(2.7$ pixels $)$ \\
d) Offset of second peak & $2.4 \mathrm{~m}$ & $1.5 \mathrm{~m}(1.8$ pixels $)$ \\
e) Level of first peak & $-61.1 \mathrm{~dB}$ & $4.3 \mathrm{~dB}(7 \%)$ \\
f) Level of second peak & $-61.3 \mathrm{~dB}$ & $4.4 \mathrm{~dB}(7 \%)$ \\
\hline
\end{tabular}

\begin{tabular}{l|rl}
\hline \multicolumn{3}{l}{ Barn 1 } \\
\hline a) Building scattering width & $13.9 \mathrm{~m}$ & $3.1 \mathrm{~m}$ (3.8 pixels) \\
b) Offset of first peak & $1.5 \mathrm{~m}$ & $0.7 \mathrm{~m}$ (0.8 pixels) \\
c) Distance between peaks & $4.9 \mathrm{~m}$ & $2.2 \mathrm{~m}$ (2.6 pixels) \\
d) Offset of second peak & $7.5 \mathrm{~m}$ & $3.4 \mathrm{~m}$ (4.1 pixels) \\
e) Level of first peak & $-65.9 \mathrm{~dB}$ & $4.3 \mathrm{~dB}(7 \%)$ \\
f) Level of second peak & $-67.2 \mathrm{~dB}$ & $8.0 \mathrm{~dB}(12 \%)$
\end{tabular}

Barn 2
a) Building scattering width
$15.5 \mathrm{~m} 2.9 \mathrm{~m}$ (3.6 pixels)
b) Offset of first peak
$1.6 \mathrm{~m} \quad 0.9 \mathrm{~m}$ (1.0 pixels)
c) Distance between peaks
$5.1 \mathrm{~m} \quad 2.9 \mathrm{~m}$ (3.6 pixels)
d) Offset of second peak
$8.7 \mathrm{~m} \quad 4.2 \mathrm{~m}$ (5.1 pixels)
e) Level of first peak
$-67.1 \mathrm{~dB} \quad 5.5 \mathrm{~dB}(8 \%)$
f) Level of second peak
$-64.5 \mathrm{~dB} \quad 10.9 \mathrm{~dB}(17 \%)$

\begin{tabular}{l|rl}
\hline \multicolumn{3}{l}{ Barn 3} \\
\hline a) Building scattering width & $13.2 \mathrm{~m}$ & $2.3 \mathrm{~m}$ (2.8 pixels) \\
b) Offset of first peak & $1.5 \mathrm{~m}$ & $0.5 \mathrm{~m}$ (0.6 pixels) \\
c) Distance between peaks & $5.6 \mathrm{~m}$ & $2.4 \mathrm{~m}$ (2.9 pixels) \\
d) Offset of second peak & $6.2 \mathrm{~m}$ & $3.3 \mathrm{~m}$ (4.0 pixels) \\
e) Level of first peak & $-60.0 \mathrm{~dB}$ & $7.0 \mathrm{~dB}(12 \%)$ \\
f) Level of second peak & $-69.3 \mathrm{~dB}$ & $8.2 \mathrm{~dB}(12 \%)$
\end{tabular}




\subsubsection{Model-based Interpretation of Features}

The two features introduced in Section 4.1.2, the roof scattering $\tilde{W}$ and the wall scattering $\tilde{H}$, contain information about the building parameters. These predicted features overlap in the image. The results of Section 4.1.1 and the known building parameters were used to interpret the observed image features in terms of the predicted features.

From the known barn parameters, the predicted image features were calculated using (4.12) and (4.11). Since all the barns appear to have the same width $W$,

$$
\tilde{W}=W \cos \psi \cos \phi \approx(14.63)(0.9129)(1)=13.36 \mathrm{~m}
$$

for all the barns.

For Barn 1,

$$
\tilde{H}=H \sin \psi \approx(3.66)(0.4081)=1.5 \mathrm{~m}
$$

and for Barns 2 and 3,

$$
\tilde{H}=H \sin \psi \approx(6.1)(0.4081)=2.49 \mathrm{~m}
$$

The PO model predicts that the wall and double bounce reflections will have a higher intensity than that from the roof because the local incident angle of the wall is greater. This is consistent with the barns signatures in the LiMIT imagery; the signature con-

sists of a narrow bright region in the near range $(\tilde{H})$ and a region of weak scattering $(\tilde{W})$ between the bright region and the darker shadow region. The observed mean to- 
tal scattering width (bright region plus weak region) was within a standard deviation of the model prediction $\tilde{W}=13.36 \mathrm{~m}$. The mean distance between the peaks, the extent of the bright region, was greater than the predicted $\tilde{H}$. This may be due to the system point spread function which spreads the bright response of the wall and double-bounce across neighboring pixels. The difference in height between the barns apparent in the test site optical image could not be inferred from the range profiles. The difference in height may be less than the height resolution given by (4.13) for LiMIT as 2 meters, or 6.6 feet.

For the L-building, at a $45 \mathrm{deg}$. aspect angle, the scattering from the roof should be stronger than that of the wall. The double-bounce scattering could be strong, depending on the ground cover (Figure 4.6). This would explain one of the bright line features observed in the L-building image. The double-bounce scattering is located in the image at the far range of the scattering from the wall $\tilde{H}$. This leads to an ambiguity as to whether the observed total scattering width corresponds to $\tilde{H}$ second peak is double-bounce - or to $\tilde{W}$ - first peak is double-bounce. Three possible building shapes consistent with the observed features are shown in Figure 4.17. 


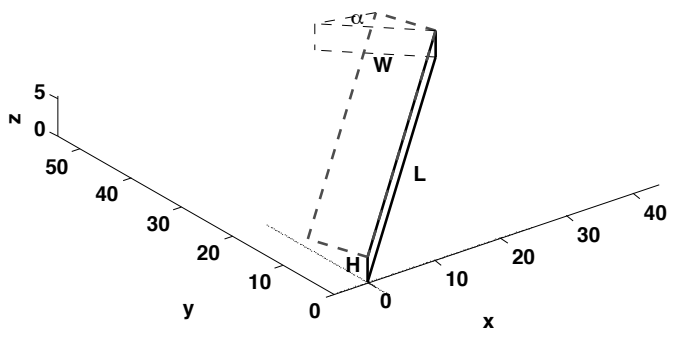

a) $\alpha=14$ deg., $H=3.5 \mathrm{~m}$

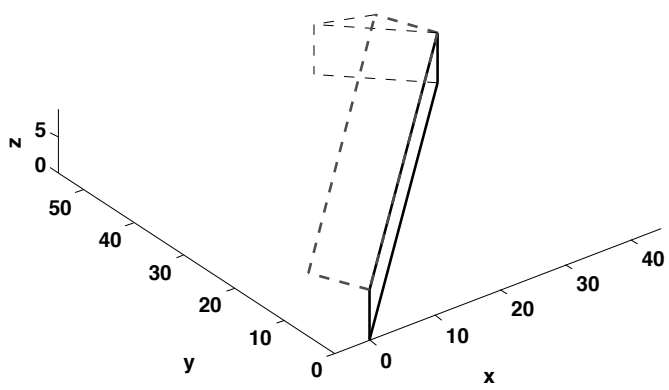

c) $\alpha=14$ deg., $H=7 \mathrm{~m}$

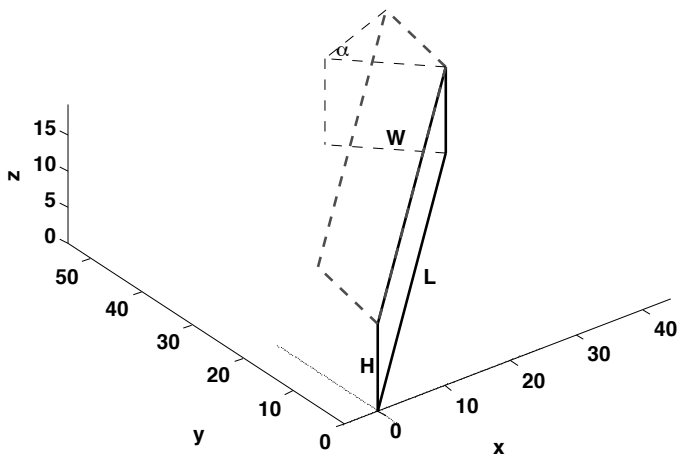

e) $\alpha=45$ deg., $H=12 \mathrm{~m}$

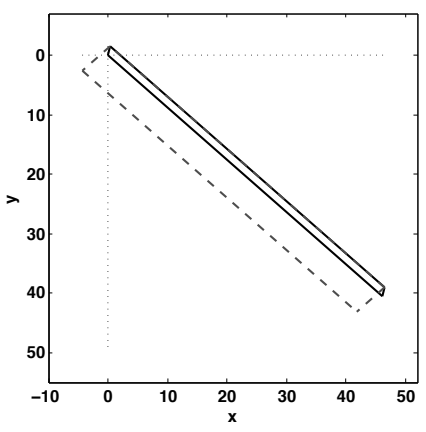

b) $\tilde{W}=8.1 \mathrm{~m}, \tilde{H}=1.8 \mathrm{~m}$

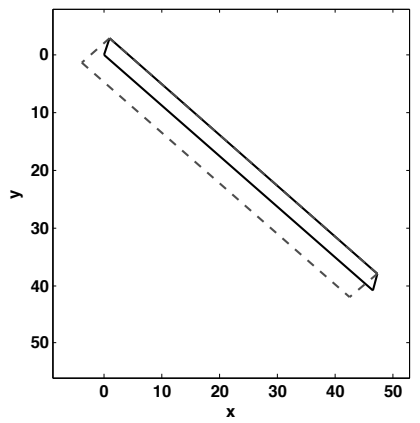

d) $\tilde{W}=8.1 \mathrm{~m}, \tilde{H}=3.5 \mathrm{~m}$

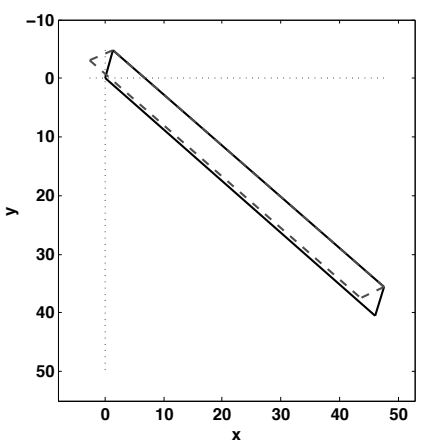

f) $\tilde{W}=5.3 \mathrm{~m}, \tilde{H}=6.1 \mathrm{~m}$

Figure 4.17: Possible L-building shapes (one wing only) are shown in (a),(c) and (e). The resulting image features for each shape are shown in (b),(d) and (f). 


\subsection{Summary}

In conclusion, the purpose of modeling the scattering intensity is to interpret the dominant features of a building signature in SAR imagery. Given the building orientation in the scene, the bright features of the building in a SAR image can be attributed to building components aided by the predictions of the scattering model. The physics-based PO scattering model is known to be accurate, but it depends on the permittivity of the building component material, which is usually not known. It was shown here that this does not limit its usefulness for the purpose of predicting whether the roof or the wall scattering will be dominant, as differences in permittivity do not change the relative intensities of the roof and the wall. On the other hand, it has been shown that the double-bounce scattering intensity is highly dependent on the roughness parameters of the ground surface. This insight is also useful for SAR image feature interpretation.

Models in the form of equations were developed that show how a building's roof and walls appear as features in a SAR image. Their usefulness for information extraction depends on the correct delineation and interpretation (e.g., roof or wall) of the image features. In practice, extracting building information from image features is difficult to apply because the image speckle noise and the system point spread function distorts the boundaries of the features. More useful are the theoretical bounds on the precision of the building parameter information contained in the features. These bounds establish a reference for evaluating the performance of an information extraction method. 


\section{Chapter 5}

\section{Simulation of SAR Signals}

SAR signal simulation was used to reproduce the observed features in the LiMIT building images using the PO scattering model, and to further investigate the sensitivity of SAR image features to changes in a building's size, roof slope and orientation. A new, efficient simulation method was developed to support the geometric accuracy and spatial coverage required for comparing the simulation images to the LiMIT imagery. The efficiency and accuracy of the simulation method makes it useful for information extraction as will be demonstrated in the next chapter.

\subsection{New SAR Simulation Method}

The size of the area imaged by a LiMIT CPI is roughly 2 kilometers by 4 kilometers with 1-meter resolution. For investigating buildings, it is sufficient to simulate a much smaller scene - say, 100 meters by 200 meters. A small scene can be simulated efficiently in the phase history (spatial frequency) domain by exploiting the inverse relationship between the bandwidth in this domain and the size of the imaged scene, 
as explained in Section 3.2. This is the basis for the new simulation method presented here, called the phase-domain simulation method. This method reconstructs the full raw time-domain signal from a reduced set of phase domain samples and requires less computation than the direct time-domain simulation method.

The time-domain simulation method calculates the raw signal using (3.13), which is the received echo from a single scatterer in the scene. The raw signal is the sum of the echos from all the scatterers in the scene,

$$
s_{m}\left(t_{n}\right)=\sum_{i=1}^{N_{s}} \gamma_{i} \exp \left[-\mathrm{j} \omega_{0} \frac{2}{c} r_{i, m}\right] \exp \left[\mathrm{j} \alpha\left(t_{n}-\frac{2}{c} r\right)^{2}\right] \operatorname{rect}\left[\frac{t_{n}-\frac{2}{c} r_{i, m}}{T}\right],
$$

where

$$
r_{i, m}=\left|\overrightarrow{\boldsymbol{R}}_{m}-\overrightarrow{\boldsymbol{r}}_{i}\right|
$$

is the distance between the $i$ the scatterer and the sensor at the time of the $m$ th pulse. The raw signal is evaluated for each sample time $t_{n}$, for each pulse return signal $s_{m}$, and for each scatterer in the scene. The number of samples recorded for each pulse is equal to the sampling rate of the sensor times the duration of the record window. The advantage of the time-domain method is that the sensor positions from an actual flight can be used, so that any deviations from an ideal flight path are captured in the simulation. Another advantage is that the simulated raw signal can be processed with the same software that is used for processing the sensor data to form an image. These two characteristics are important when the goal is to compare the simulation to data. The disadvantage of the time-domain method is that it is computationally intensive making it impractical for high-resolution simulations of detailed scenes.

The phase-domain method works as follows (see Figure 5.1): First, the surface reflectivity for a scene is specified using an appropriate scattering model. Second, the phase 


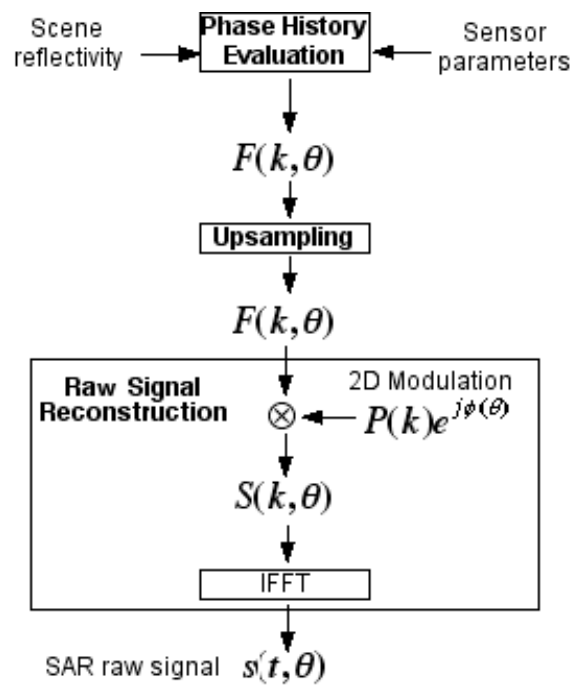

Figure 5.1: Phase-domain simulation method.

history is evaluated at a reduced sampling rate based on the scene dimensions and the sensor parameters. Third, the phase history is upsampled to the full frequency domain sampling rate of the sensor data phase history. Finally, the demodulation process described in Section 3.2 is applied in reverse to reconstruct the raw signal. This method scales well because only the first step increases in computation time with the size of the scene; the other steps are fixed for a given set of sensor parameters.

The phase-domain method exploits the relationship between the range dimension length of the final image $D_{y}$ and the phase history sample spacing $\mathrm{d} k_{y}$,

$$
D_{y}=\frac{2 \pi}{\mathrm{d} k}
$$

This inverse relationship means that a smaller image is produced by a larger sample spacing and, therefore, a smaller number of samples. An example illustrates the computational savings. Using (5.3), a scene that is 200 meters long requires a phase history sample spacing of approximately $750 \mathrm{kHz}$. Using (3.27), a resolution of 1 
meter requires a bandwidth of $150 \mathrm{MHz}$ in the phase history domain. Then the number of phase history samples per pulse required to form a 1-meter resolution image 200 meters long is $150 \mathrm{MHz} / 750 \mathrm{kHz}=200$ samples. This is a small number compared to the 36,800 samples per pulse of the LiMIT time-domain raw signal, which is sampled at $480 \mathrm{MHz}$ for $76 \mu \mathrm{sec}$. Herein lies the key advantage of the phase-domain simulation method - reducing the number of samples evaluated greatly reduces the computation time to produce the full raw time-domain signal.

\subsubsection{Phase History Evaluation}

The phase-domain simulation method begins by evaluating the phase history $F(k, \theta)$ using the sensor parameters and the input scene. The sensor parameters needed for the simulation are summarized in Table 5.1. The input scene consists of a discrete set of scatterers each described by a position $\overrightarrow{\boldsymbol{r}}$ and a reflectivity $\gamma$. The expression for the phase history of a single point in the scene was given by (3.19); the phase history for the input scene is calculated by summing this expression over all the scatterers in the scene,

$$
F\left(k, \theta_{m}\right)=\sum_{i}^{N_{s}} \gamma_{i} \exp \left[\mathrm{j} k r_{i, m}\right] \operatorname{rect}\left[\frac{k-k_{0}}{4 \alpha T / c}\right]
$$

where $N_{s}$ is the number of scatterers in the scene.

The key to this step is the selection of the discrete values of $k$ and $\theta$ at which to evaluate the phase history $F(k, \theta)$. To see why this is important, consider the goal of comparing the simulation image to a SAR image. The pixels of the two images must be the same size and have the same coordinates. To achieve this, the simulation phase history must be evaluated at a subset of the frequencies and view angles of the 
Table 5.1: Sensor Parameters For Simulation

\begin{tabular}{cc} 
Parameter & Symbol \\
\hline Sensor position for each pulse & $\overrightarrow{\boldsymbol{R}}_{m}$ \\
Number of pulses & $N_{p}$ \\
Center frequency & $f_{0}$ \\
Chirp rate & $2 \alpha$ \\
Pulse duration & $T$ \\
Record window duration & $T_{r}$ \\
Sampling rate & $f_{s}$ \\
Number of points for FFT & $N_{\mathrm{FFT}}$
\end{tabular}

sensor data phase history.

The phase history samples of the processed data are at frequencies determined by the sampling rate of the raw signal and the number of points used for the FFT to transform the recorded signal from each pulse,

$$
f_{n}=\frac{-f_{s}}{2}+(n-1) \frac{f_{s}}{N_{\mathrm{FFT}}}, \quad n=1: \frac{N_{\mathrm{FFT}}}{2} .
$$

The sample spacing, converting to spatial frequency, is

$$
\mathrm{d} k=\frac{2}{c} 2 \pi \frac{f_{s}}{N_{\mathrm{FFT}}} .
$$

The maximum phase history sample spacing required for the simulation is calculated using (5.3),

$$
\mathrm{d} k^{\prime}=\frac{2 \pi}{D_{y}}
$$

where $D_{y}$ is calculated from the input scene. The simulation phase history (5.4) is then evaluated at the discrete values of $k$ corresponding to a subset of the frequencies 
of $(5.5)$,

$$
k_{n}=\frac{2}{c} 2 \pi f_{n}, \quad n=1:\left\lfloor\frac{\mathrm{d} k^{\prime}}{\mathrm{d} k}\right\rfloor: \frac{N_{\mathrm{FFT}}}{2} .
$$

Similarly, the subset of the view angles $\theta$ at which to evaluate the simulation phase history is selected using,

$$
D_{x}=\frac{2 \pi}{\mathrm{d} \theta}
$$

where $D_{x}$ is the cross-range length of the final image and $\mathrm{d} \theta$ is the angular spacing of the view angles of each pulse.

The next step before reconstructing the raw signal is to upsample $F(k, \theta)$ to match the sample spacing of the sensor data phase history in the $k$ dimension. This is accomplished by applying a one-dimensional FFT in the $k$ dimension, zero-padding the resulting transform signal, and then applying an inverse FFT.

The resulting upsampled signal includes the values of the signal at the original sample locations plus interpolated values between them, as shown in Figure 5.2. This is why it was important to choose the subset of frequencies as described at the beginning of this section, so that the upsampled signal has values corresponding to the frequencies of the sensor data phase history.

\subsubsection{Raw Signal Reconstruction}

To reconstruct the raw signal, the upsampled phase history is subjected to the reverse of the demodulation process described in Section 3.2 and then transformed into the 


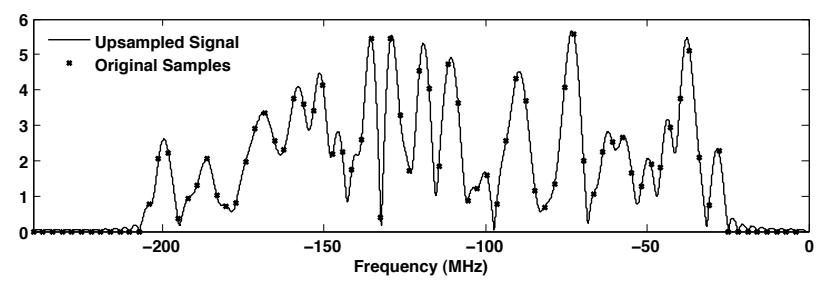

Figure 5.2: The upsampled signal contains the original sample locations, with additional interpolated samples inserted.

time-domain.

The reverse of the demodulation process is a two-dimensional modulation. The modulation function in the $k$ dimension is the transform of the transmitted pulse, $P_{0}(k)$. The modulation function in the $\theta$ dimension is a phase shift due to the difference in the distance to the scene center for each pulse position.

After modulation, the signal is

$$
S\left(k, \theta_{m}\right)=F\left(k, \theta_{m}\right) P_{0}(k) \exp \left[\mathrm{j} k\left(r_{0}-R_{m}\right)\right]
$$

The final step is to perform an inverse Fourier transform into the time-domain. The raw signal is a real-valued signal; since the transform of a real signal is conjugate symmetric, the symmetry is introduced prior to the inverse transform,

$$
\begin{gathered}
S_{m}(\omega)=\left\{\begin{aligned}
S\left(\frac{2}{c} \omega, \theta_{m}\right) & : 0 \leq \omega<2 \pi f_{s} / 2 \\
S^{*}\left(\frac{2}{c} \omega-2 \pi f_{s} / 2, \theta_{m}\right) & : 2 \pi f_{s} / 2 \leq \omega<2 \pi f_{s}
\end{aligned}\right. \\
s_{m}\left(t_{n}\right)=\operatorname{IFFT}\left\{S_{m}(\omega)\right\}
\end{gathered}
$$

If the raw signal recorded by the sensor is zero-padded prior to FFT, i.e., $N_{\mathrm{FFT}}>N_{t}$, 


\begin{tabular}{rrrr}
\multicolumn{4}{c}{ Test Scene } \\
$x$ & $y$ & $z$ & $\gamma$ \\
\hline 0 & 0 & 0 & 3 \\
0 & 0 & 50 & 5 \\
-50 & 100 & 0 & 3 \\
100 & -50 & 0 & 3 \\
-100 & 50 & 50 & 5
\end{tabular}

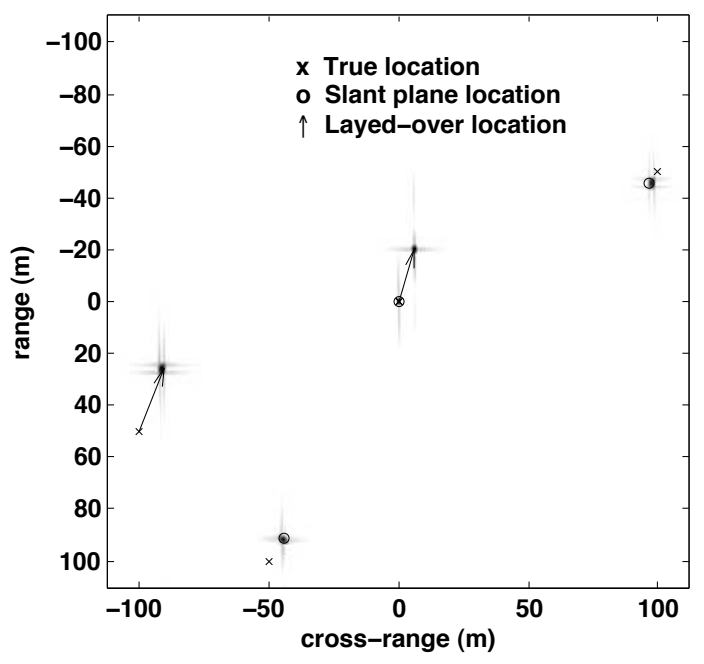

Figure 5.3: The final image of the test scene verifies the simulation. The image was produced by processing the simulated raw signal with the LiMIT processing software. The slant plane image locations of the input scene points were calculated using the mapping equations (4.6) and (4.7).

then the reconstructed raw signal will be zero-padded as well and the extra values are discarded. Finally, $s_{m}\left(t_{n}\right)$ should be scaled and typed to match the actual data. For example, the LiMIT data is recorded as 8-bit signed integers so the values of the reconstructed signal are scaled to the range [-128 127] and converted to 8 bits. The final image of Figure 5.3 was produced by processing the reconstructed signal with the same code used to process the actual LiMIT data.

The reconstructed raw signal was compared to the time-domain simulation of the same scene. The time-domain simulated raw signal was generated by evaluating (5.1). The difference between the raw time-domain simulated sample values and the reconstructed sample values was on the order of 10e-14, which can be attributed entirely to computer round-off errors. Therefore, the phase-domain simulation method presented here produces the same result as the time-domain method and there is no trade-off between computational efficiency and accuracy. 
Table 5.2: Computational Cost Factors

\begin{tabular}{ll}
\hline \multicolumn{1}{c}{ Time-Domain Method } \\
\hline$N_{s}$ & scatterers in scene \\
$N_{p}$ & pulses \\
$N_{t}$ & raw signal samples per pulse \\
\hline \multicolumn{2}{c}{ Phase-Domain Method } \\
\hline$N_{s}$ & scatterers in scene \\
$N_{p}$ & pulses \\
$N_{f}^{\prime}$ & phase history samples evaluated \\
$N_{f}$ & phase history samples per pulse
\end{tabular}

\subsubsection{Computational Cost}

The computational cost of an algorithm can be quantified as the number of complex multiplies required by the algorithm. The factors that determine the number of complex multiplies required by the time-domain simulation method and the phasedomain method are listed in Table 5.2.

The number of multiplies required by the time-domain simulation method is

$$
N_{\mathrm{TD}}=N_{s} N_{p} N_{t}
$$

The number of multiplies required by the new phase-domain method is

$$
N_{\mathrm{PD}}=N_{s} N_{p} N_{f}^{\prime}+N_{r}
$$

where $N_{r}$ is the number of multiplies required for the upsampling and reconstruction. The cost of the upsampling and reconstruction is

$$
N_{r}=N_{p}\left[2 N_{f} \log _{2} N_{f}+N_{f}+N_{f} \log _{2} N_{f}\right]
$$


where the first term in the brackets is the cost of the FFTs ${ }^{1}$ used for upsampling, the second term is the cost of the modulation, and the third term is the cost of the final inverse FFT.

The computational cost of the phase-domain method relative to the time-domain method is

$$
\frac{N_{\mathrm{PD}}}{N_{\mathrm{TD}}}=\frac{N_{f}^{\prime}}{N_{t}}+\frac{N_{r}}{N_{s} N_{t} N_{p}}
$$

Simplifying and substituting into 5.16,

$$
\begin{aligned}
\frac{N_{\mathrm{PD}}}{N_{\mathrm{TD}}} & =\frac{N_{f}^{\prime}}{N_{t}}+\frac{N_{r}}{N_{s} N_{t} N_{p}} \\
& =\frac{N_{f}^{\prime}}{N_{t}}+\frac{N_{p} N_{f}\left(3 \log _{2} N_{f}+1\right)}{N_{s} N_{t} N_{p}} \\
& =\frac{N_{f}^{\prime}}{N_{t}}+\frac{N_{f}\left(3 \log _{2} N_{f}+1\right)}{N_{s} N_{t}}
\end{aligned}
$$

The first term on the right-hand side is typically very small. The second term decreases with the number of scatterers; this is because the cost of the upsampling and reconstruction does not depend on the number of scatterers.

An example illustrates the reduction in computation. Consider a scene 100 meters by 200 meters scene divided into 0.5 meter by 0.5 meter square scatterers so $N_{s}=$ 80,000. Using the LiMIT parameters for $N_{t}$ and $N_{f}$, the computation required by the

\footnotetext{
${ }^{1}$ The number of multiplies used by an FFT algorithm is bounded by $N \log _{2} N$ where $N$ is the number of points evaluated [72]
} 
phase-domain method relative to the time-domain method is

$$
\begin{aligned}
\frac{N_{\mathrm{PD}}}{N_{\mathrm{TD}}} & =\frac{200}{36800}+\frac{24567\left(3 \log _{2}(24567)+1\right)}{(80000)(36800)} \\
& =0.0054+\frac{1.1 \mathrm{e}+06}{2.94 \mathrm{e}+10} \\
& =0.005437 .
\end{aligned}
$$

For a more practical frame of reference, on a desktop computer with dual $\operatorname{Intel}(\mathrm{R})$ Xeon(TM) $2.40 \mathrm{GHz}$ processors the simulation example above takes over 12 hours using the time-domain method and just over 30 minutes using the new phase domain method.

\subsection{Simulation Results}

The phase-domain simulation method was used to reproduce the observed features in the LiMIT building images, and to further investigate the sensitivity of SAR image features to changes in a building's size, roof slope and orientation.

The input to the simulation is a three-dimensional scene. The coordinate system of the scene is defined with the origin at the center, and the scene has length $D_{x}$ meters in the $x$ (cross-range) dimension and length $D_{y}$ meters in the $y$ (range) dimension. The scene is composed of equal-sized facets that are described by an $x-y-z$ location and a complex reflectivity $\gamma$. The magnitude of the reflectivity value is the radar cross section (RCS) which is calculated using the PO model described in Section 4.1.1. The phase of the reflectivity of each facet is random, distributed uniformly between 0 and $2 \pi$. 


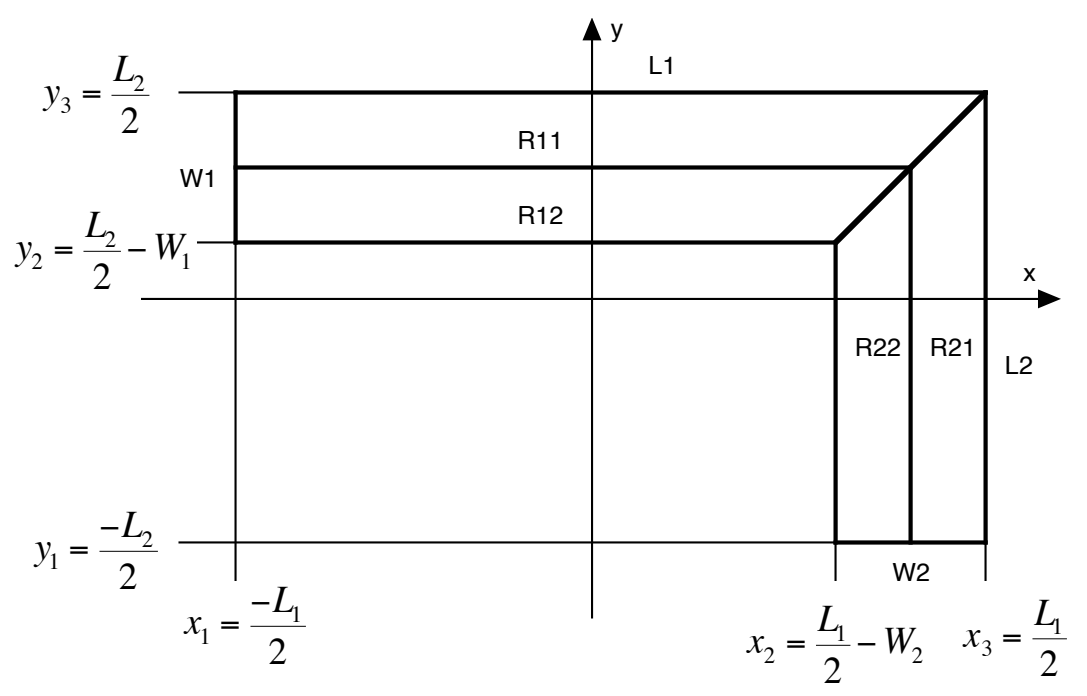

Figure 5.4: Simulated building layout. The plain type labels indicate named regions of the building that are referred to in the text.

The facet locations are spaced evenly in the $x-y$ plane, where the spacing in each dimension is half the final image resolution $\rho_{x}$ and $\rho_{y}$ determined by the LiMIT parameters. This results in four facets per resolution cell (pixel), each with random phase so that the characteristic SAR speckle noise is reproduced.

\subsubsection{Simulation of Buildings}

The scene is composed of a building and ground. The building layout within the scene is shown in Figures 5.4 and 5.5. The building has two wings (L-shaped) if both $\mathrm{W} 1$ and $\mathrm{W} 2$ are non-zero; if one of $\mathrm{W} 1$ or $\mathrm{W} 2$ is zero, then the building has a single wing (rectangular, like the barns). Once the scene is constructed, it is rotated in the $x-y$ plane by an angle $\phi$ which is the building orientation in the scene.

The scene is layed out so that each facet belongs exclusively to the ground, one of the vertical walls, or one of the roof sections. There are a total of nine of these 


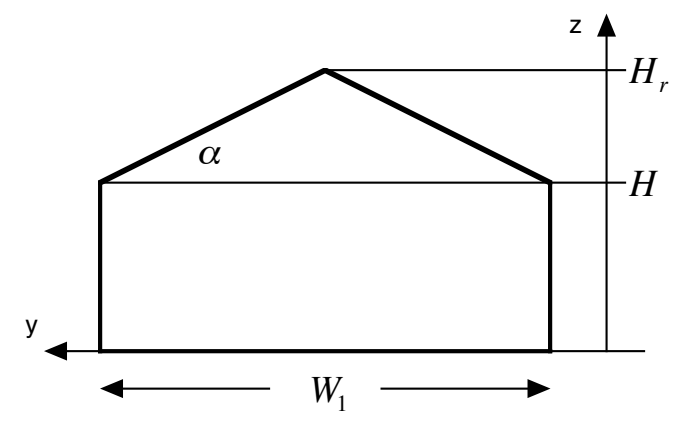

Figure 5.5: Simulated building, side-view. The angle of the roof $\alpha$ is determined by the height of the roof peak, the height of the vertical walls and the length of the building side.

scene components. Each component is assigned a permittivity and a normal vector; the ground is also assigned roughness statistics, $\sigma$ and $\ell_{c}$. The incident angle 4.5 is calculated for each of the nine normal vectors using the sensor pointing vector 4.2. The RCS of each component is calculated using the PO model. The facets at the base of each wall are assigned the RCS of the double-bounce scattering using the GO-PO model. If the incident angle of a component is greater than 90 degrees, then facets belonging to that component are not illuminated and their reflectivity is set to zero. The non-illuminated facets are still created so that the correct shadowing effects are included, and then removed from the scene after the shadow calculation. Two example input scenes are shown in Figure 5.6, an L-shaped building with two wings and a single wing building like the barns.

A simulation of a barn is shown in Figure 5.7 along with the LiMIT image of the barn. The simulation reproduces the features observed in the LiMIT image — bright strip from wall and double-bounce, region of weak scattering from the roof, and shadow.

A simulation of an L-shaped building is shown in Figure 5.8. The simulation does not reproduce the parallel lines observed in the LiMIT image. The PO scattering model predicts that the scattering from the vertical walls will be weak for a building 


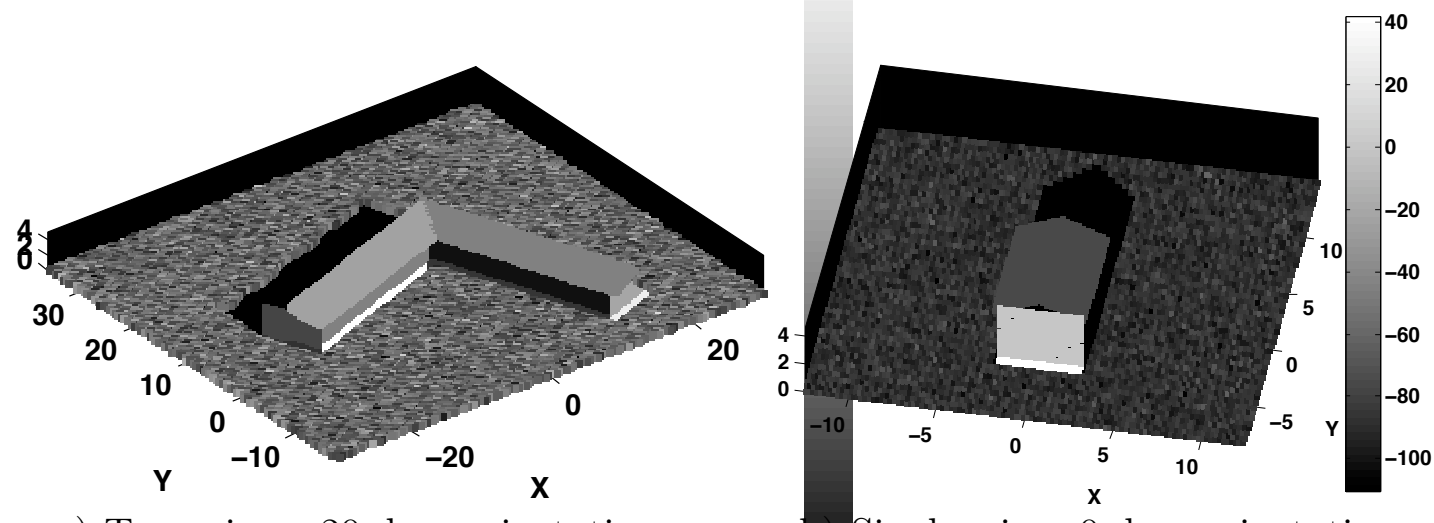

a) Two wings, 20 deg. orientation. $\quad$ b) Single wing, 0 deg. orientation.

Figure 5.6: Example synthetic building scenes. The sensor location relative to the scene is uprange in the negative $y$ direction. The bright facets at the base of the walls are assigned the intensity of the double-bounce scattering.

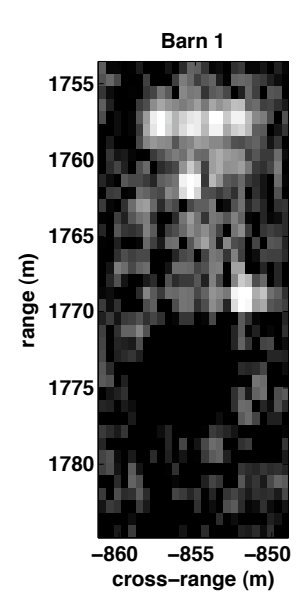

a) LiMIT image of barn.

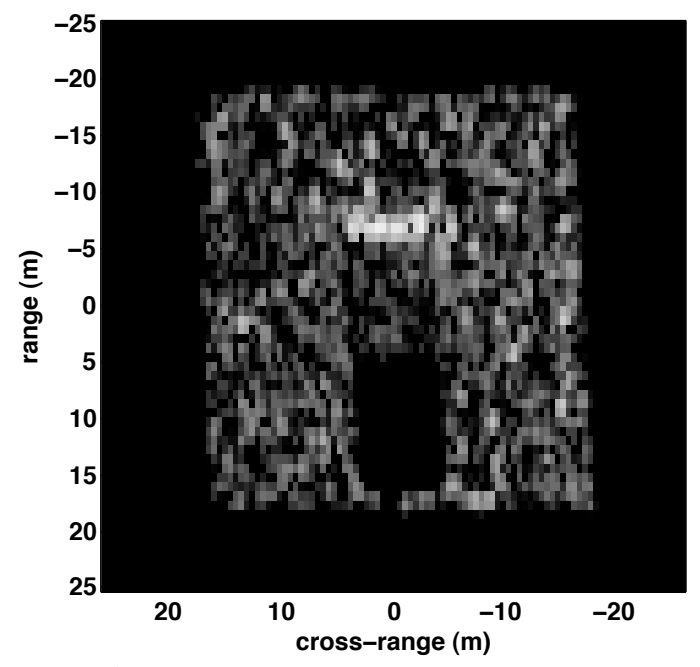

b) Simulation of barn.

Figure 5.7: The simulation of the barn captures the signature observed in the LiMIT image - bright strip from wall and double-bounce, region of weak scattering from the roof, and shadow. 


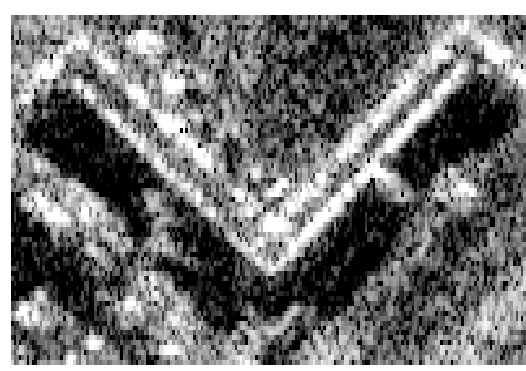

a) LiMIT image of L-building.

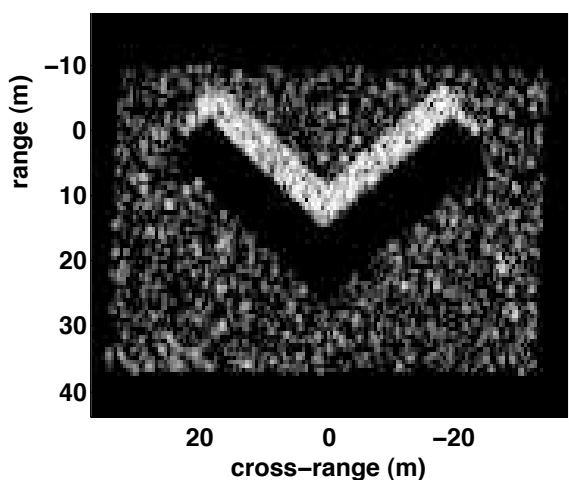

b) Simulation of L-building.

Figure 5.8: Simulation of a scaled down version of the L-building. The parameters for both wings were $\mathrm{L}=32 \mathrm{~m}, \mathrm{~W}=8 \mathrm{~m}, \mathrm{H}=4 \mathrm{~m}$, and $\alpha=14 \mathrm{deg}$.

orientation of 45 degrees, like that of the L-building. The double-bounce scattering could be strong if the building is surrounded by asphalt and so one of the bright lines may be this component. But it is not clear from the model why the roof produces a second bright line. Simulations of a single wing of the building are shown in Figure 5.9, where the double-bounce scattering intensity was calculated for asphalt and appears very bright. A possible explanation for the second bright line is that the roof contains an architectural detail not accounted for in the building shape model. Another possible explanation is that the observed bright line is due to diffraction by the wedge shape of the roof ridge.

\subsubsection{Evaluation of Building Feature Model}

The relationship between the building parameters and the image features was given in Section 4.1.2 and are repeated here for convenience,

$$
\tilde{H}=H\left[\sin \psi-\cos \psi \sin \varphi \tan \tilde{\phi}_{1}\right]
$$




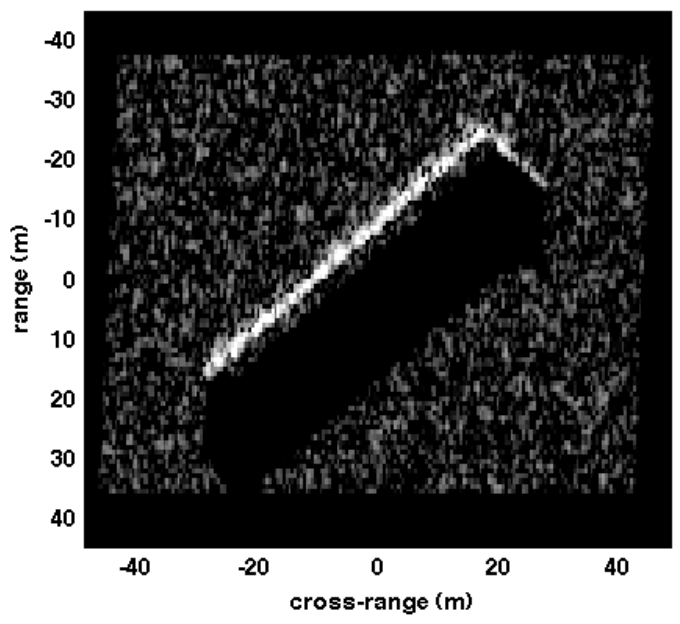

a) $\mathrm{L}=63 \mathrm{~m}, \mathrm{~W}=15 \mathrm{~m}$, $\mathrm{H}=7 \mathrm{~m}, \alpha=14 \mathrm{deg}$.

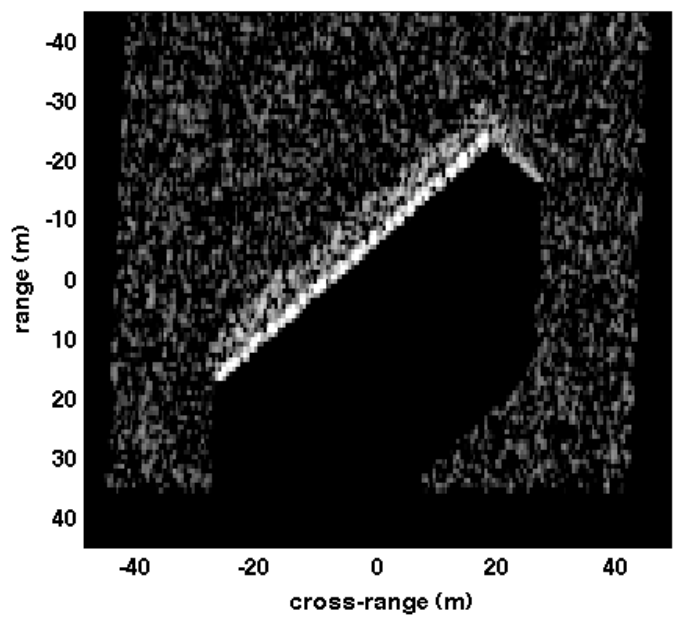

b) $\mathrm{L}=63 \mathrm{~m}, \mathrm{~W}=12 \mathrm{~m}$, $\mathrm{H}=15 \mathrm{~m}, \alpha=40 \mathrm{deg}$.

Figure 5.9: Simulation of one wing of the L-building using two different hypothesized shapes. The bright line is the double-bounce at the base of the wall. For the low-sloped roof (a), the roof scattering feature is wider than the wall scattering feature. For the steep-sloped roof (b), the opposite is the case.

$$
\begin{aligned}
\tilde{W}= & (W / 2)[|\cos \phi \cos \psi-\tan \alpha \sin \psi| \\
& \left.+(\sin \phi \cos \varphi+\cos \phi \sin \psi \sin \varphi+\tan \alpha \cos \psi \sin \varphi) \tan \tilde{\phi}_{1}\right]
\end{aligned}
$$

where $\tilde{W}$ is the width of the roof scattering and $\tilde{H}$ is the width of the wall scattering in the image.

To investigate the image feature associated with the scattering from the vertical wall, a scene was created that contained a single vertical wall. No rough ground (background facets) were included so that there would be no ambiguity as to which pixels in the image should be assigned to the wall feature. The magnitude of the reflectivity of the wall facets was calculated using the PO model and the phase of the reflectivity was drawn from a uniform distribution between 0 and $2 \pi$. The length of the wall was 10 $\mathrm{m}$ in the cross-range dimension. The height and orientation of the wall was varied. For each height and orientation, a simulation was generated and an image was formed 


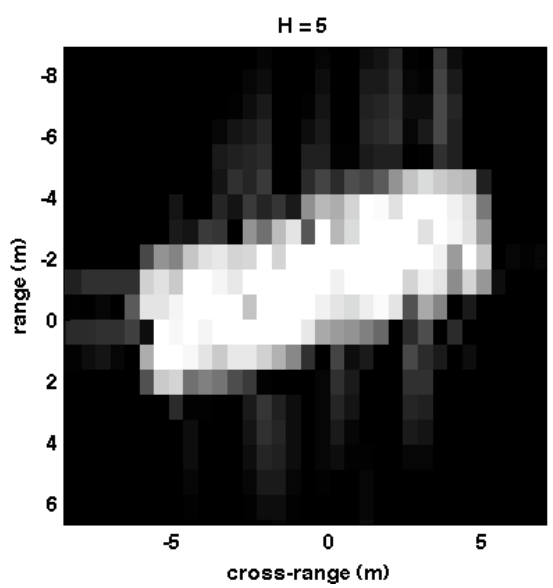

Figure 5.10: Simulation image of a vertical wall on a non-reflective background. The height of the wall was $5 \mathrm{~m}$ and the orientation angle is $20 \mathrm{deg}$. The intensity of the wall feature varies due to the random phase of the reflectivity. The point response of each facet is a $2 \mathrm{D}$ sinc function.

from the simulated signal.

An example image is shown in Figure 5.10. The intensity of the image varies over the region corresponding to the wall even though each wall facet was assigned the same reflectivity magnitude. The variation in pixel intensity arises from the random phase of the reflectivity, which produces speckle noise. The image shows energy from the wall spread into pixels corresponding to the non-reflective background. This is due to the point spread function of (3.25).

These two effects, random phase speckle noise and the point spread function, blur the edges of the wall feature, even with the absence of background scattering. To measure the width of the wall feature, the number of pixels having an intensity above a threshold were counted in each range column. The threshold was calculated for each image as $75 \%$ of the maximum intensity in the image. The width of the wall feature for a particular image was calculated as the mean number of pixels in each range column multiplied by the pixel width in the range dimension. The results are 
Table 5.3: Simulation Wall Feature

\begin{tabular}{|c|c|c|c|c|c|c|c|}
\hline$H(\mathrm{~m})$ & $\begin{array}{c}\text { rientation: } \\
\text { Predicted } \\
\tilde{H}(\mathrm{~m})\end{array}$ & $\begin{array}{c}0 \text { deg } \\
\text { Measured } \\
\tilde{H}(\mathrm{~m})\end{array}$ & Diff. & $\begin{array}{l}20 \text { deg } \\
\text { Measured } \\
\tilde{H}(\mathrm{~m})\end{array}$ & Diff. & $\begin{array}{l}40 \text { de } \\
\text { Measured } \\
\tilde{H}(\mathrm{~m})\end{array}$ & Diff. \\
\hline 4.0 & 1.64 & 1.77 & 0.13 & 2.27 & 0.63 & 3.23 & 1.59 \\
\hline 4.5 & 1.84 & 2.71 & 0.87 & 2.76 & 0.91 & 2.69 & 0.85 \\
\hline 5.0 & 2.05 & 2.79 & 0.74 & 2.59 & 0.54 & 2.98 & 0.93 \\
\hline 6.0 & 2.46 & 3.64 & 1.18 & 2.99 & 0.54 & 3.27 & 0.81 \\
\hline 8.0 & 3.27 & 4.28 & 1.00 & 4.12 & 0.84 & 4.53 & 1.26 \\
\hline 8.5 & 3.48 & 3.78 & 0.30 & 4.42 & 0.94 & 4.59 & 1.11 \\
\hline 9.0 & 3.68 & 3.69 & 0.01 & 4.33 & 0.65 & 4.65 & 0.97 \\
\hline 10.0 & 4.09 & 3.71 & -0.38 & 5.25 & 1.16 & 5.17 & 1.08 \\
\hline
\end{tabular}

Note: The pixel size of the simulation image was 0.8165 . The Measured $\tilde{H}$ is the mean value from all the range profiles in a single simulation image.

given in Table 5.3. The model predicts that the width of the wall feature is unaffected by the wall orientation angle; the results for three different orientation angles support this. In all but one case, the measured feature is wider than the model predicts. The difference is less than one pixel width in 10 out of 24 cases, and less than two pixel widths in all cases. This suggests that the threshold value used to identify the pixel values may have been too low, so that extra pixels were included in the count.

The same procedure was repeated for the image roof feature $\tilde{W}$, where the input to the simulation was a scene composed of a sloped roof over a non-reflective background. The length of the roof $L$ was $10 \mathrm{~m}$ and the height of the lowest part of the roof $H$ was $8 \mathrm{~m}$. The width $W$ and the slope angle $\alpha$ were varied since these are the parameters that determine the width of the roof scattering in the image. The results are shown in Table 5.4 as the difference between the measured $\tilde{W}$ and that predicted by the model. As with the wall feature, the measured feature width was greater than the model predicted width. In 30 out of 48 cases, the difference was less than one pixel width and all were less than two. 


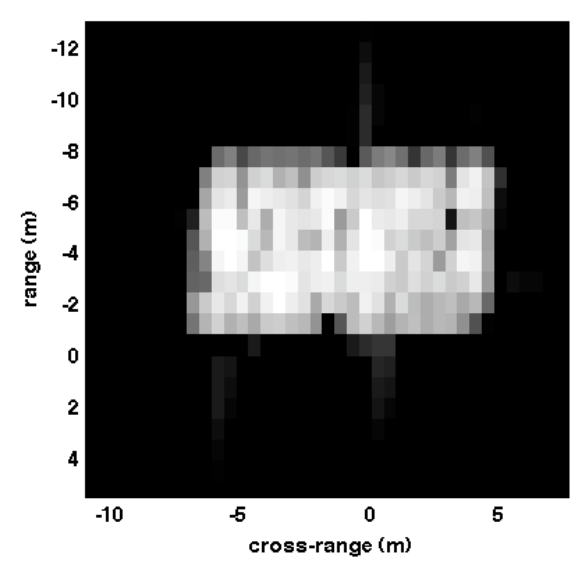

a. $23 \mathrm{deg}$. slope angle.

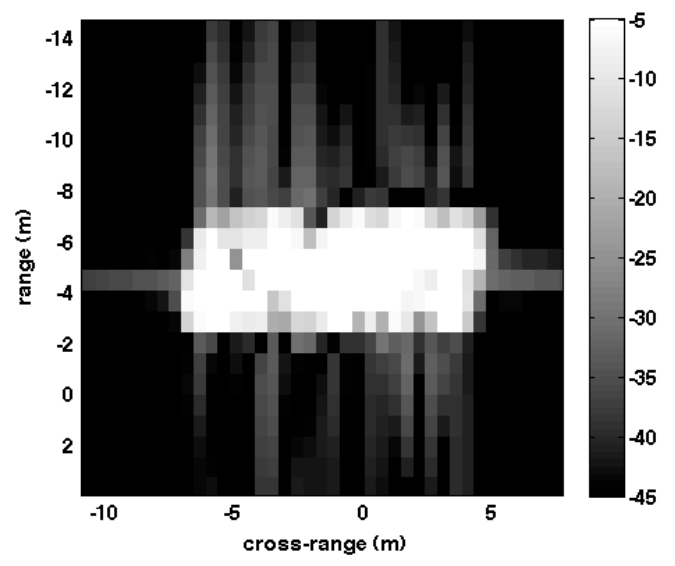

b. 45 deg. slope angle.

Figure 5.11: Simulation images of a sloped roof on a non-reflective background. The height of the roof was $8 \mathrm{~m}$ at the lowest edge, the length was $10 \mathrm{~m}$, and the width was $6 \mathrm{~m}$. The effective incident angle for the $23 \mathrm{deg}$. roof (a) was $43 \mathrm{deg}$. and for the $45 \mathrm{deg}$ roof (b) the incident angle was $21 \mathrm{deg}$. The increased steepness of the roof compresses the roof feature in the image and increases the intensity of the scattering.

Table 5.4: Simulation Roof Feature

\begin{tabular}{r|rccccccrr}
\multicolumn{10}{c}{ Measured $\tilde{W}$ - Predicted $\tilde{W}$} \\
$W(\mathrm{~m})$ & 0 & 7 & 14 & 23 & 27 & 34 & 45 & 56 \\
\hline 5 & 0.58 & 0.73 & 0.68 & 0.37 & 1.02 & 0.53 & 1.04 & 0.50 \\
6 & 0.62 & 0.82 & 1.26 & 1.04 & 0.91 & 0.61 & 0.51 & 0.69 \\
7 & 0.86 & 0.91 & 1.41 & 0.97 & 0.99 & 0.66 & 0.92 & 0.80 \\
8 & 0.76 & 0.73 & 0.32 & 1.02 & 0.25 & 0.01 & 0.78 & 1.01 \\
9 & 0.70 & 0.58 & 0.88 & 0.80 & 1.04 & 0.71 & 0.65 & 0.68 \\
10 & 0.81 & 0.37 & 0.39 & 0.26 & 0.17 & 0.92 & 0.14 & 0.01 \\
& Note: The pixel size of the image was 0.8165.
\end{tabular}




\subsection{Summary}

The phase domain simulation method is a computationally efficient method for simulating a region on the order of 100 square meters at the full resolution of the SAR sensor data. The phase of the complex reflectivity of an illuminated structure plays a role in the appearance of the structure in the SAR image. The effect of the phase speckle noise - is reproduced by simulating the raw signal and then forming an image using the same processing chain that is used for the sensor data.

The relationship between the building parameters - wall height, roof slope and width - and the image features was verified using the simulation. It was found that the model underestimates the width of the features in the image by one or two resolution cells. This observed excess width of the features was due to the point spread function and speckle noise. The reliability of the range profile method for feature extraction depends on the length of the feature in the cross-range dimension. The longer the feature, the more range profile lines are available for measurement which means a larger sample size for estimating the mean feature width.

The simulation was used to reproduce the building signatures observed in the LiMIT data. For the barns, the simulation agrees qualitatively with the observed signature. For the L-building, the parallel bright lines are not reproduced. This indicates limitations of the underlying building model, which does not include architectural details or diffraction scattering. 


\section{Chapter 6}

\section{Mutual Information Method For Information Extraction}

The relationship between a building's shape and the SAR image features it produces was established in Chapter 4 but it is difficult to extract information directly from features. This is because the image features overlap and their edges are blurred by the speckle and point spread effects demonstrated in Chapter 5. An alternative is to use simulation to find the hypothetical building shape that produces a SAR response most similar to that observed in the SAR image. The similarity between a simulation image and a SAR image is quantified using mutual information. Maximizing the mutual information between the two images is equivalent to maximizing the likelihood of the SAR image given the hypothesized building shape. 


\subsection{Theoretical Considerations}

An image $v$ is a two-dimensional matrix of intensity values, and the values are distributed according to some probability density function (pdf) $\mathcal{P}(\mathrm{v})$. A building is specified by parameter vector,

$$
\boldsymbol{b} \doteq[W, L, H, \alpha, \phi]
$$

where the parameters are those defined in Figure 4.1. Let $\mathrm{v}_{0}$ be the SAR image of a building and let $\boldsymbol{v}_{\boldsymbol{b}}$ be the simulation image produced using $\boldsymbol{b}$.

The shape of a building is estimated as that which maximizes the mutual information between the SAR image and the simulation image,

$$
\boldsymbol{b}^{*}=\underset{\boldsymbol{b}}{\arg \max }\left\{\mathcal{I}\left(\mathrm{v}_{0}, \mathbf{v}_{\boldsymbol{b}}\right)\right\}
$$

The mutual information of (6.2) is related to the log likelihood of the SAR image $v_{0}$ given the simulation image $\mathbf{v}_{\boldsymbol{b}}$,

$$
\begin{aligned}
\mathcal{I}\left(\mathrm{v}_{0}, \mathrm{v}_{\boldsymbol{b}}\right) & =\mathcal{H}\left(\mathrm{v}_{0}\right)-\mathcal{H}\left(\mathrm{v}_{0} \mid \mathrm{v}_{\boldsymbol{b}}\right) \\
& =\mathcal{H}\left(\mathrm{v}_{0}\right)-\left(-\sum_{\mathrm{v}_{\boldsymbol{b}}} \mathcal{P}\left(\mathrm{v}_{\boldsymbol{b}}\right) \sum_{\mathrm{v}_{0}} \mathcal{P}\left(\mathrm{v}_{0} \mid \mathrm{v}_{\boldsymbol{b}}\right) \log \mathcal{P}\left(\mathrm{v}_{0} \mid \mathrm{v}_{\boldsymbol{b}}\right)\right) \\
& =\mathcal{H}\left(\mathrm{v}_{0}\right)-\left(-\sum_{\mathrm{v}_{\boldsymbol{b}}, \mathrm{v}_{0}} \mathcal{P}\left(\mathrm{v}_{0}, \mathrm{v}_{\boldsymbol{b}}\right) \log \mathcal{P}\left(\mathrm{v}_{0} \mid \mathrm{v}_{\boldsymbol{b}}\right)\right) \\
& =\mathcal{H}\left(\mathrm{v}_{0}\right)+E_{\mathrm{v}_{0}, \mathrm{v}_{\boldsymbol{b}}}\left[\log \mathcal{P}\left(\mathrm{v}_{0} \mid \mathrm{v}_{\boldsymbol{b}}\right)\right]
\end{aligned}
$$

where the second term on the right is the expectation over the joint distribution of 
the $\log$ likelihood. The entropy of the SAR image $\mathcal{H}\left(\mathrm{v}_{0}\right)$ is fixed, so maximizing the mutual information is equivalent to maximizing the expectation of the log likelihood of the data given the model.

\subsubsection{Probability Distribution Estimation}

The entropy of an image is a function of the probability distribution of the image pixel values. The most common way to estimate the pixel value distribution is to use a histogram of the pixel values [51]. The histogram is a nonparameteric consistent estimator [73], although the choice of bin width is critical to the quality of the estimate. If the bin width is too wide, then the histogram is overly smoothed; if the bin width is too narrow, then the bins may be sparsely filled and the resulting density estimate is unreliable.

The bin width $w$ can be chosen explicitly or it can be chosen implicitly by specifying the number of bins. Oftentimes, the number of bins is chosen based on the number of bits used to record the data, e.g., 128 bins for 8-bit data. Sturges' Rule [74] specifies the number of bins as

$$
N_{\text {bin }}=\left\lceil 1+\log _{2} N_{\text {pixel }}\right\rceil
$$

where $N_{\text {pixel }}$ is the number of pixels in the image. The bin width is then

$$
w=\frac{\max v-\min v}{N_{\text {bin }}} .
$$

In effect, this rule says that the number of data points (pixels) determines the precision with which the pdf can be reliably estimated. 
Other rules that are used in the statistics community are Scott's Rule [75],

$$
w=\left\lfloor\frac{3.5 \sigma}{N_{\text {pixel }}^{1 / 3}}\right\rfloor,
$$

where $\sigma$ is the standard deviation of the pixel intensity values, and Freedman-Diaconis' Rule,

$$
w=\frac{2 \mathrm{IQR}}{N_{\text {pixel }}^{1 / 3}},
$$

where IQR is the interquartile range ${ }^{1}$ of the pixel intensity values.

Sturge's Rule is not optimal in all situations [76], but it has been demonstrated empirically $[77,60]$ to perform better than the other rules for calculating the mutual information between two images. As an example, each of the rules is applied to the Lbuilding image and the resulting histograms are shown in Figure 6.1. As the number of bins decreases, the bin width increases and the histogram becomes less sparse. The entropy also decreases with the number of bins.

The application of Sturge's Rule results in only 15 bins, a far smaller number than the other rules. The sufficiency of 15 bins to describe the information of the image is illustrated in Figure 6.2. The image on the left is displayed using 128 levels of gray and the image on the right using 15. They appear indistinguishable to the human eye. A smaller number of bins decreases the memory and computation required for calculating the entropy and mutual information.

\footnotetext{
${ }^{1}$ The interquartile range is a statistical measure of dispersion. It is the difference between the 75 th percentile and the 25 th percentile.
} 
Sensor Dynamic Range (128 bins)

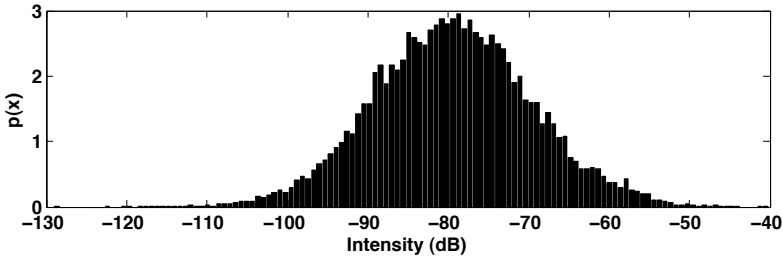

bin width $w$

$0.69 \mathrm{~dB}$

$\%$ non-empty

89

entropy $\mathcal{H}(\mathrm{v}) \quad 5.87$

Freedman-Diaconis' Rule (85 bins)

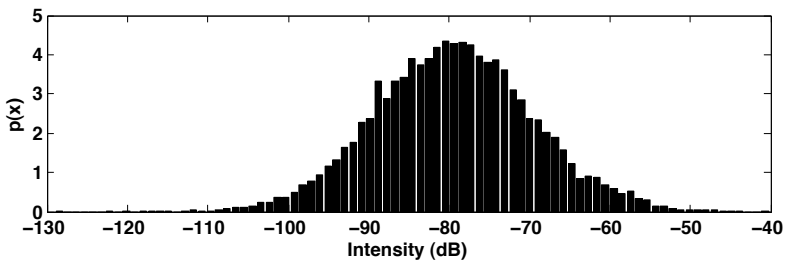

bin width $w$

$1.04 \mathrm{~dB}$

$\%$ non-empty

Scott's Rule (64 bins)

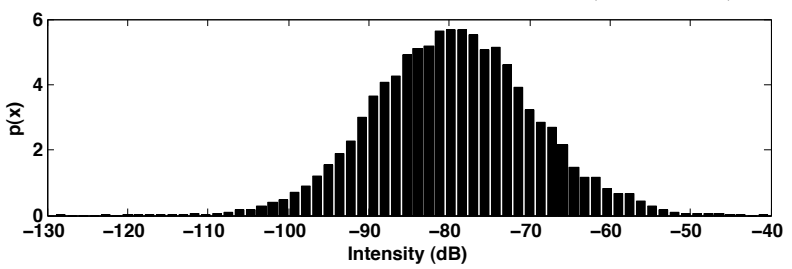

bin width $w$

$1.39 \mathrm{~dB}$

$\%$ non-empty

92

entropy $\mathcal{H}(\mathrm{v})$

4.88

Sturge's Rule (15 bins)

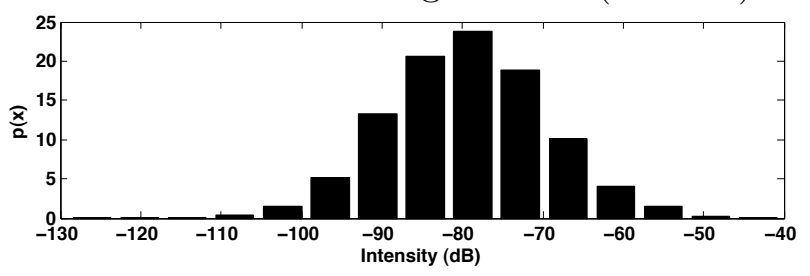

bin width $w$

$5.92 \mathrm{~dB}$

$\%$ non-empty

100

entropy $\mathcal{H}(\mathrm{v})$

2.81

Figure 6.1: Effect of bin width on pdf estimate and calculated entropy. The data are the pixel intensity values of the L-building image. 


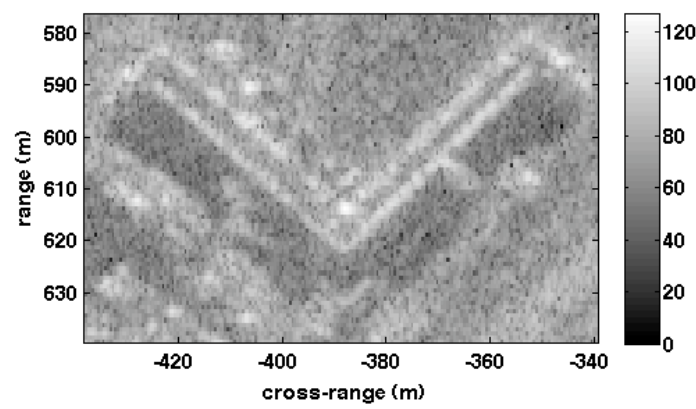

a) 128 bins

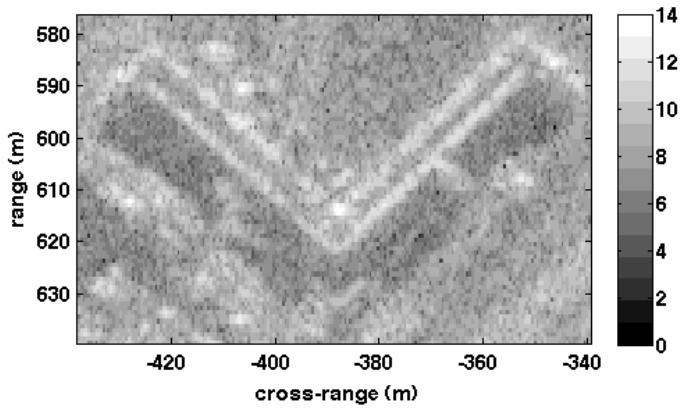

b) 15 bins

Figure 6.2: The same image viewed using two different intensity bin widths. The original intensity values were mapped to the range 1 to $N_{\text {bins }}$ and each bin corresponds to a unique gray-level. The human eye cannot discern a difference in the detail present.

\subsubsection{Mutual Information Normalization}

To compare the mutual information between a SAR image and different simulation images, the mutual information measure must be normalized to remove the dependence on the absolute entropy values of the images. The entropy of each simulation image may be different, and the simulation image entropy affects the bound on the mutual information between the simulation image and the SAR image,

$$
0 \leq \mathcal{I}\left(\mathrm{v}_{0}, \mathrm{v}_{\boldsymbol{b}}\right) \leq \min \left[\mathcal{H}\left(\mathrm{v}_{0}\right), \mathcal{H}\left(\mathrm{v}_{\boldsymbol{b}}\right)\right]
$$

For example, consider two simulation images, $v_{b 1}$ and $v_{b 2}$, such that

$$
\mathcal{H}\left(\mathrm{v}_{\boldsymbol{b} 1}\right)<\mathcal{H}\left(\mathrm{v}_{\boldsymbol{b} 2}\right)<\mathcal{H}\left(\mathrm{v}_{0}\right)
$$

Then $\mathcal{I}\left(\mathrm{v}_{0}, \mathrm{v}_{\boldsymbol{b} 1}\right)$ has less range than $\mathcal{I}\left(\mathrm{v}_{0}, \mathrm{v}_{\boldsymbol{b} 2}\right)$. The two mutual information measures must be scaled to the same range. This is accomplished here by dividing the mutual 
information by the entropy of the simulation image,

$$
\begin{aligned}
\mathcal{I}^{\prime}\left(\mathrm{v}_{0}, \mathrm{v}_{\boldsymbol{b}}\right) & \doteq \frac{\mathcal{I}\left(\mathrm{v}_{0}, \mathrm{v}_{\boldsymbol{b}}\right)}{\mathcal{H}\left(\mathrm{v}_{\boldsymbol{b}}\right)} \\
& =\frac{\mathcal{H}\left(\mathrm{v}_{\boldsymbol{b}}\right)-\mathcal{H}\left(\mathrm{v}_{\boldsymbol{b}} \mid \mathrm{v}_{0}\right)}{\mathcal{H}\left(\mathrm{v}_{\boldsymbol{b}}\right)} \\
& =1-\frac{\mathcal{H}\left(\mathrm{v}_{\boldsymbol{b}} \mid \mathrm{v}_{0}\right)}{\mathcal{H}\left(\mathrm{v}_{\boldsymbol{b}}\right)}
\end{aligned}
$$

This measure is a value between 0 and 1 . The minimum value of 0 occurs when $\mathcal{H}\left(\mathbf{v}_{\boldsymbol{b}} \mid \mathrm{v}_{0}\right)$ is equal to $\mathcal{H}\left(\mathrm{v}_{\boldsymbol{b}}\right)$ which occurs when the SAR image and the simulation image are independent. The maximum value of 1 occurs when $\mathcal{H}\left(\mathbf{v}_{\boldsymbol{b}} \mid \mathrm{v}_{0}\right)$ is 0 which occurs when the simulation image exactly matches the SAR image.

\subsection{Application to LiMIT Data}

The mutual information method was applied to the buildings introduced in Section 4.2.1, the L-building and the barns. It was not possible to quantify the error of the resulting estimates due to the lack of precise ground truth. However, a qualitative assessment of the reasonableness of the estimates was made based on the available optical imagery and on a general knowledge of buildings. The consistency of the estimates was evaluated by using four different examples of each building from four different LiMIT CPIs. 


\subsubsection{Methodology}

Selection of SAR image. The SAR image was a manually selected region of a LiMIT image containing a single building - the L-building or one of the barns. The region was selected so that the entire building and its shadow were contained in the region while minimizing the background contained in the region.

Selection of candidate building parameters. The parameters $H$ and $\alpha$ were varied, with the others remaining fixed at the values determined from the optical imagery (see Section 4.2, Table 4.5 and Table 4.6).

The range of values for $H$ and $\alpha$ were selected based on a priori knowledge of buildings. The height $H$ of a building is greater than one meter (3.28 feet), and generally greater than two meters (6.56 feet). According to the Council on Tall Buildings and Urban Habitat $(\mathrm{CTBUH})^{2}$, a value of 3.5 meters (11.48 feet) per story is used to calculate the exterior height of a building when the type of the building, residential or commercial, is unknown. Using this value, the height of a 4-story building would be 14 meters (49 feet). The slope of the roof $\alpha$ of a building can vary from 0 degrees (flat) to very steep (greater than 50 degrees). Common roof slopes found in the United States were given in Table 4.1.

The step size of the parameter values used in the simulations was chosen based on the resolution of these parameters in the LiMIT data. The resolution of $H$ and $\alpha$ was quantified in (4.16 and (4.18). As discussed in Section 4.1.2, resolution is defined as the minimum difference in the parameters that could be determined from an image. The difference is determined by the resolution of the image, i.e. the pixel size, and

\footnotetext{
${ }^{2}$ www.ctbuh.org
} 
by the change in the image feature produced by a change in the building parameter. For example, if the resolution of $H$ is 2 meters, then a change in $H$ of less than 2 meters produces a change in $\tilde{H}$ that is less than 1 image pixel and therefore is not detectable.

Simulation. Simulations were generated using the phase-domain simulation method presented in Chapter 5. Since the LiMIT parameters were used for the simulation, the resulting simulation images had the same pixel size as the SAR image and the two images could be compared without sub-pixel interpolation or resampling. The simulation input scene contained a building, specified by the parameters $\boldsymbol{b}$, and surrounding ground. The scene was made much larger than the building so that the resulting simulation image contained all the layed-over building features as well as the shadow. The simulation image was larger than the SAR image.

Calculation of mutual information. The mutual information between the SAR image and a particular simulation image was calculated as

$$
\mathcal{I}^{\prime}\left(\mathrm{v}_{0}, \mathrm{v}_{\boldsymbol{b}}\right)=\max _{\tau} \mathcal{I}^{\prime}\left(\mathrm{v}_{0}, \mathrm{v}_{\boldsymbol{b}, \tau}\right)
$$

where $\tau$ specifies a region of the simulation image that is the same size as the SAR image. The region is specified by the row and column of the upper left pixel of the region. An exhaustive search was performed by first selecting the region located in row 1, column 1 and then shifting the region one pixel at a time in each direction. This effected an alignment, or registration, of the SAR image with the simulation image.

The mutal information at each step was calculated from the joint histogram of the 
SAR image and the selected region of the simulation image. The number of bins and the bin width of the histogram were chosen according to Sturges' Rule given in (6.7) and (6.8). The pixel intensity values of each image were binned separately according to the dynamic range of the image.

$$
i(\tilde{\gamma})=\left\lfloor\frac{\tilde{\gamma}-\min v}{\max v-\min v}\left(N_{\mathrm{bin}}-1\right)\right\rfloor+1
$$

where $i(\tilde{\gamma})$ is the index of the bin assigned to pixel value $\tilde{\gamma}$.

The joint histogram has $N_{\text {bin }}^{2}$ bins to represent every possible pairing of the $N_{\text {bin }}$ indices of the two images. The joint probability distribution was estimated from the joint histogram,

$$
\hat{\mathcal{P}}\left(\mathrm{v}_{0}, \mathrm{v}_{\boldsymbol{b}, \tau}\right)=\frac{h(i, j)}{N_{\text {pixel }}^{2}},
$$

where $h(i, j)$ is the joint histogram count of the number of times that a pixel in one image has the bin index $i$ when the same pixel in the other image has the bin index $j$. The marginal probabilities were also estimated from the joint historgram,

$$
\hat{\mathcal{P}}\left(\mathrm{v}_{0}\right)=\frac{1}{N_{\text {pixel }}} \sum_{j} h(i, j),
$$

and

$$
\hat{\mathcal{P}}\left(\mathrm{v}_{\boldsymbol{b}, \tau}\right)=\frac{1}{N_{\text {pixel }}} \sum_{i} h(i, j) .
$$

The mutual information was then calculated using (3.35).

Similarity map. The final result is a mapping from building parameter space $\boldsymbol{b}$ to mutual information $\mathcal{I}$, the similarity measure. The mapping was used to identify $\boldsymbol{b}^{*}$ from (6.2), and to evaluate the behavior of the mutual information measure as a 
function of building parameters.

\subsubsection{Case 1: L-Building}

The selected regions from each CPI are shown in Figure 6.3. The L-bldg is large in terms of pixels; each region is 198 x 85 pixels in cross-range and range, respectively. The resolution of the height parameter $H$ was calculated using (4.16) to be 1.62 meters (5.32 feet). The resolution of the roof angle parameter $\alpha$ was calculated using (4.18) to be 12.7 degrees. The candidate values for $H$ ranged from 4 to 40 feet in increments of 4 feet. The candidate values for $\alpha$ were those of Table 4.1. Note that the step size for both the parameters was less than their calculated resolution.

An example of the registration step is illustrated in Figure 6.4. The map on the right shows the mutual information as a function of translational shifts of the SAR image relative to the simulation image. Local maxima are evident that correspond to misalignments. This is why an exhaustive search was used to find the best alignment.

Figure 6.5, which shows the resulting similarity maps for each CPI. The results were consistent across the four CPIs; not only was the location of the maximum the same, but the general shape of the similarity surface was also consistent. The parameter values that maximized the mutual information were $H=20$ feet and $\alpha=7.1$ degrees. These values are reasonable, indicating a two-story building with a low-slope roof. The similarity maps indicate that a flat roof and a very steep roof are less likely.

The mutual information similarity measure varied smoothly with the parameter values. It is worth noting the diagonal ridge in the shape of the function. This is due 
CPI 1

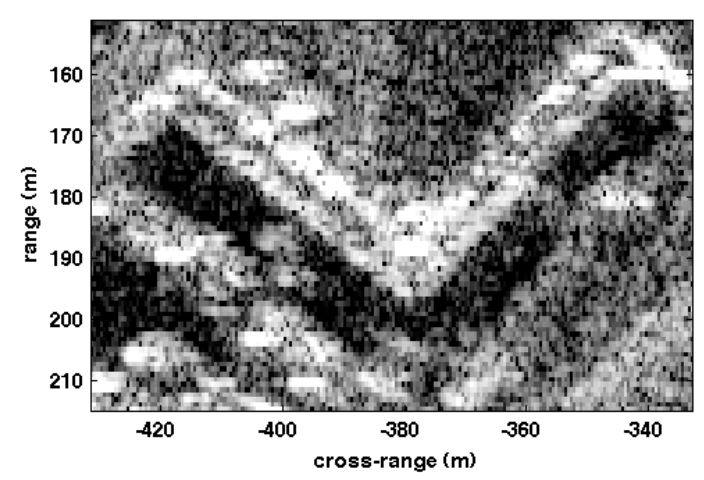

CPI 3

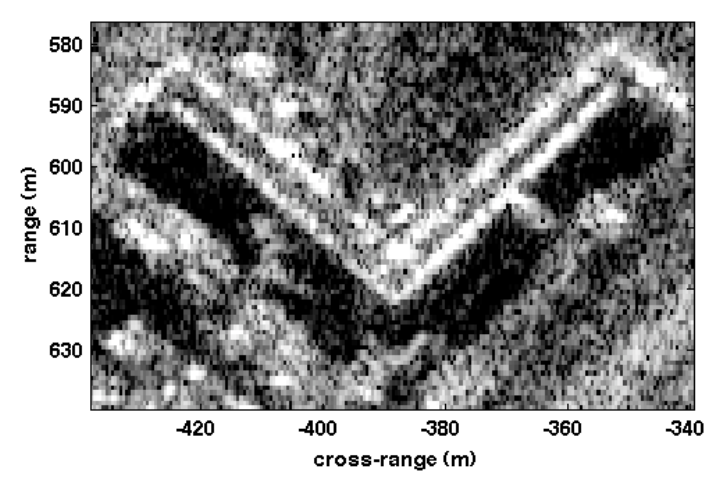

CPI 2

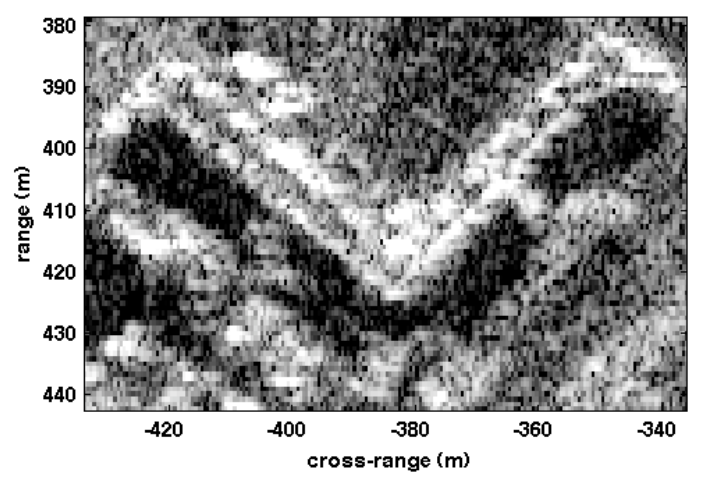

CPI 4

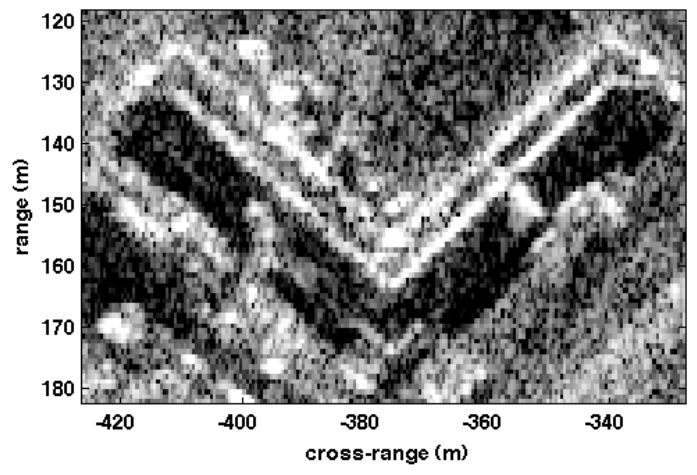

Figure 6.3: LiMIT image regions used for reference SAR images of the L-building.

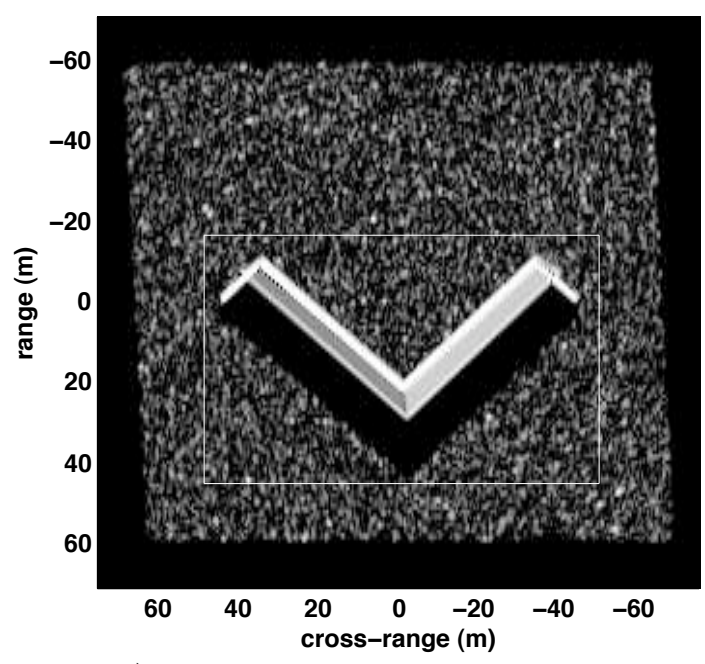

a) Simulation image $\boldsymbol{v}_{\boldsymbol{b}}$.

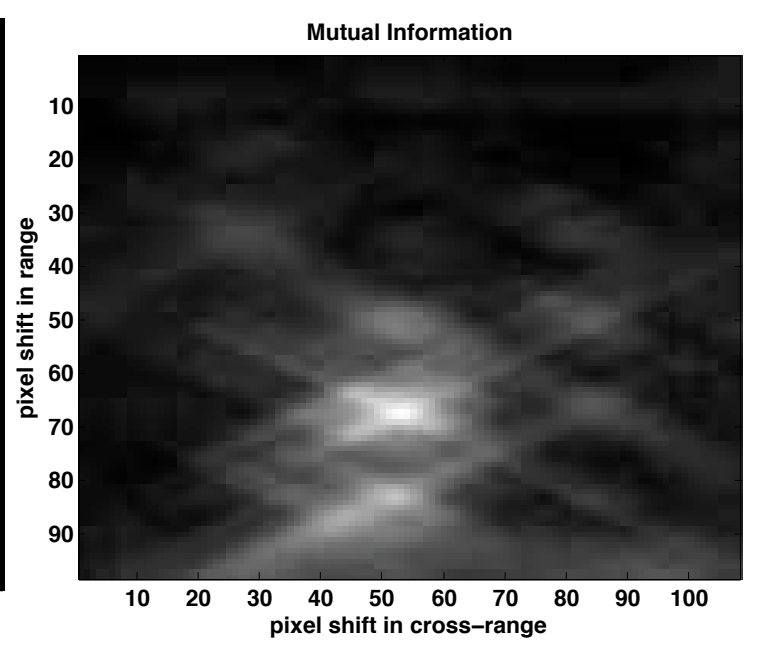

b) $\mathcal{I}^{\prime}\left(\mathrm{v}_{0}, \mathrm{v}_{\boldsymbol{b}, \tau}\right)$

Figure 6.4: The white box on the simulation image (a) indicates the region location $\tau$ that maximized the mutual information function (b). 
to the coupling of the wall height and roof angle in determining the roof peak height of the building; a building with short walls and a steep roof, and a building with high walls and a low-sloped roof could both have the same roof peak height. This ambiguity was noted by Thiele [20], who pointed out that in both cases the total width of the building scattering and the length of the shadow would be the same in the SAR image. To overcome the ambiguity, Thiele used the interferometric phase as an additional source of information. The results here suggest that the mutual information measure could also resolve this ambiguity using a single SAR intensity image.

\subsubsection{Case 2: Barns}

The LiMIT image regions used for the barns are shown in Figure 6.6. The three barns are much smaller in pixels than the L-building; the average size of the regions was 25 x 35 pixels. There is evidence of other strong scatterers - vehicles? - very close to the barns. The orientation of the barns is such that the roof ridge is parallel to the pointing vector of the sensor so that the scattering width of the roof $\tilde{W}$ is independent of the roof angle $\alpha$. The resolution of the building height $H$ was calculated to be 2 meters $(6.5$ feet $)$.

For the first experiment, only the height parameter $H$ was varied. The roof angle parameter $\alpha$ was held constant at 14 degrees, based on the optical photos that show that the barns have low sloped roofs. The height was varied from 1 to 7 meters, in increments of 1 meter. The upper limit was determined by simulation using heights up to 10 meters; a height greater than 7 meters produced a shadow that extended beyond the region matched to the LiMIT image region, as shown in Figure 6.7a. For 


\section{CPI 1}

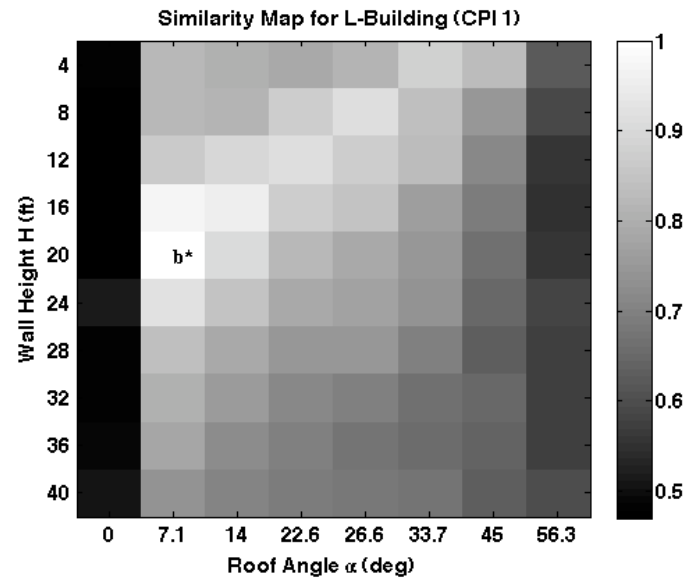

CPI 3

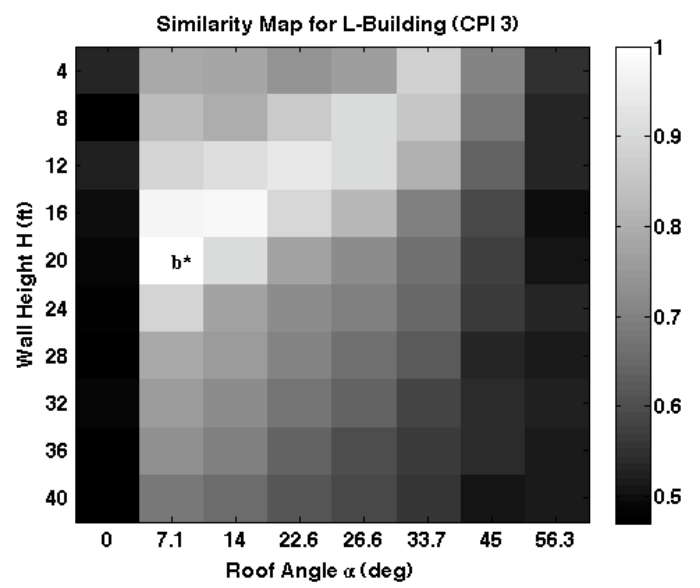

\section{CPI 2}

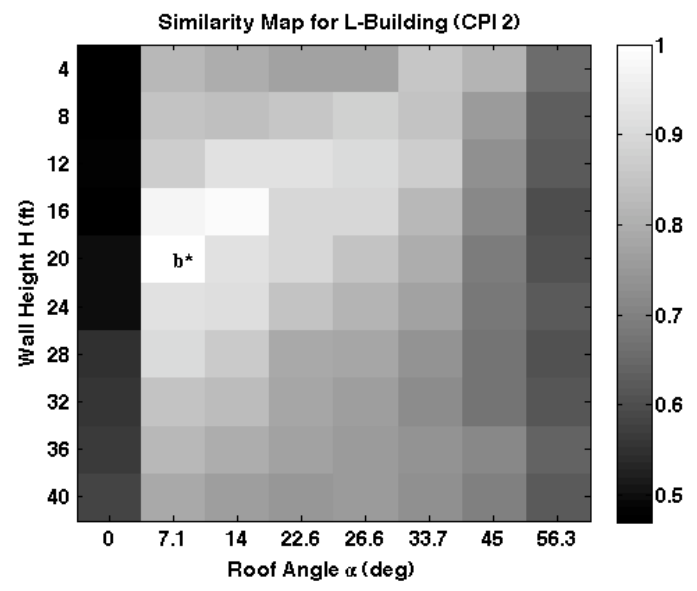

CPI 4

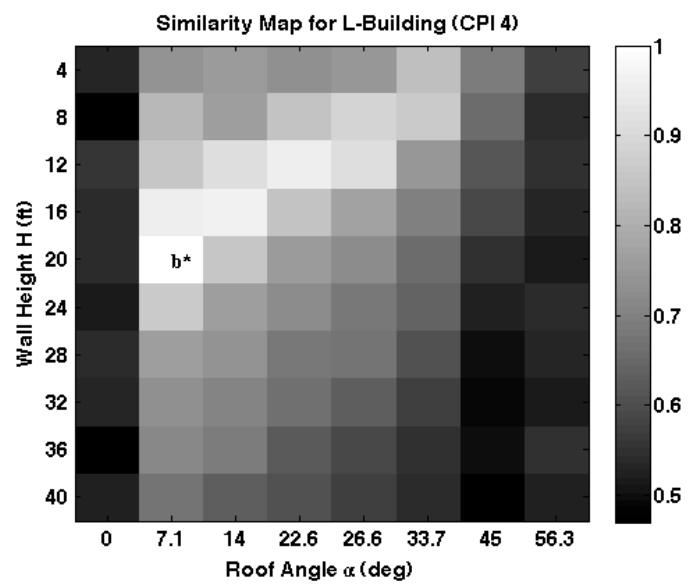

Figure 6.5: Similarity maps for the L-building. 

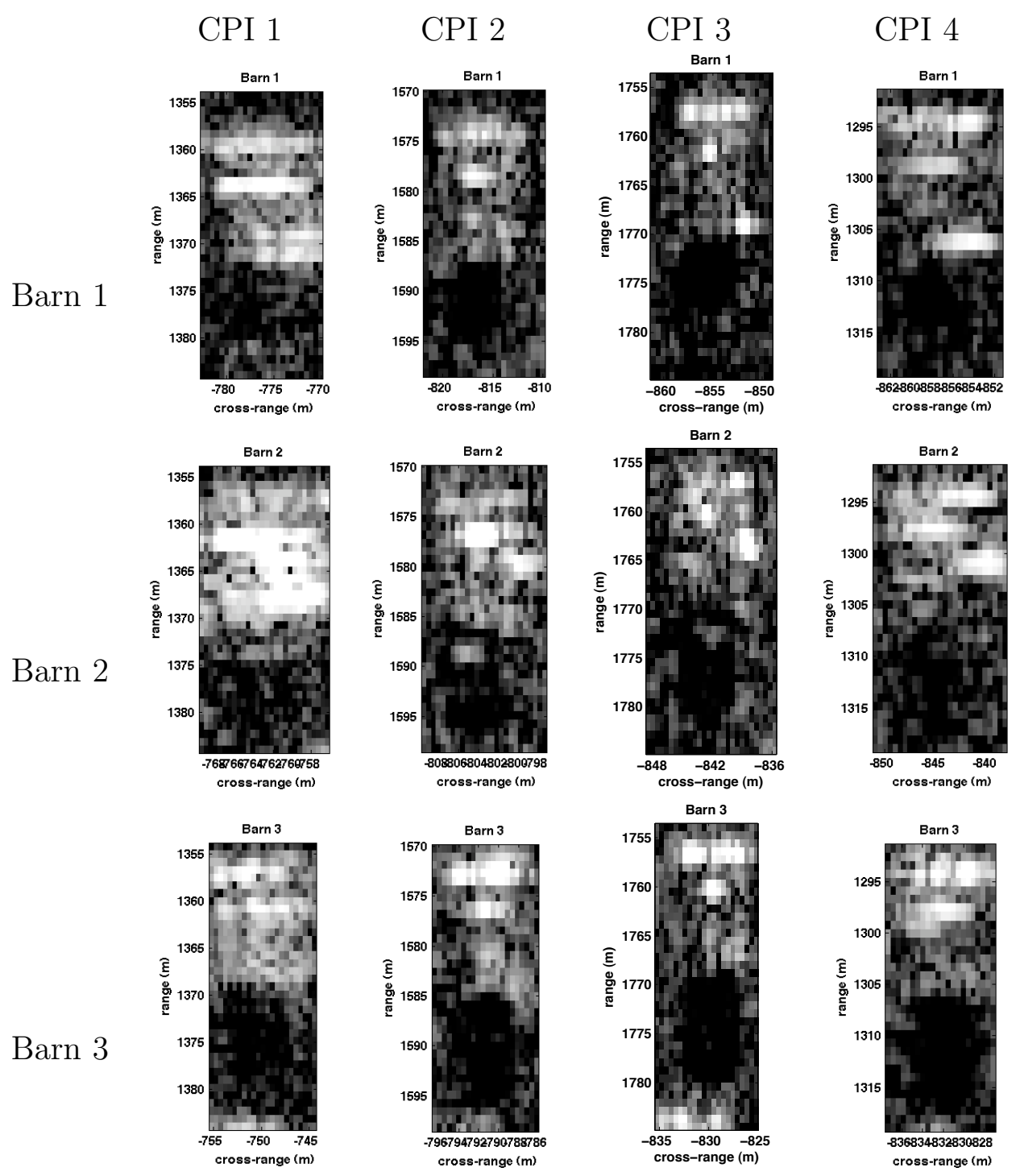

Figure 6.6: The LiMIT image regions used for the reference SAR images of the barns. 


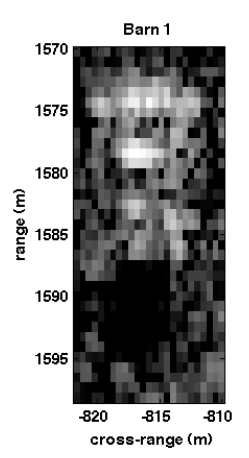

a. SAR image.

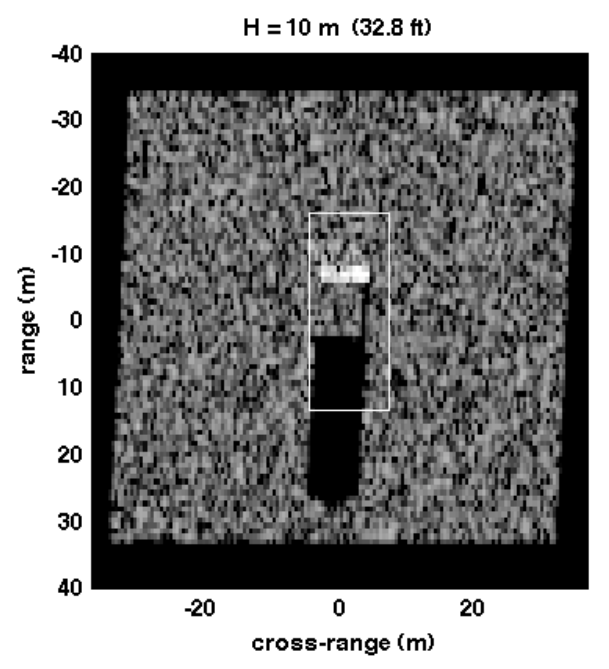

b. Tall barn.

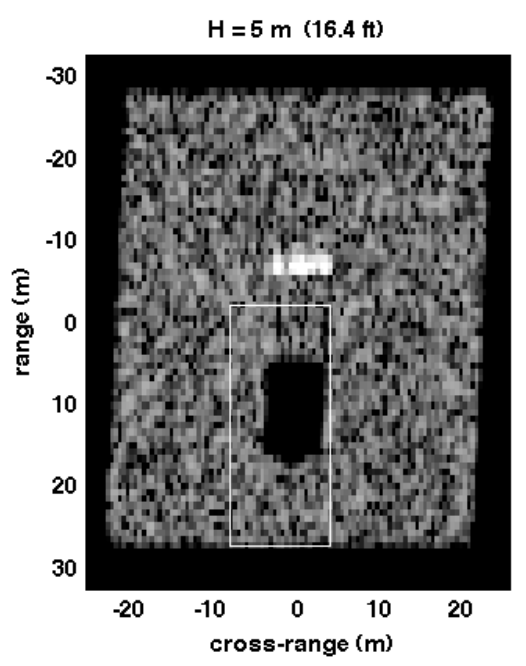

c. Misalignment.

Figure 6.7: Examples of the registration step for Barn 1. The white boxes indicate the region location $\tau$ that maximized the mutual information between the simulation image and the SAR image. b) The region does not contain the whole shadow, so this barn shape was rejected. c) The maximum mutual information produced a misalignment.
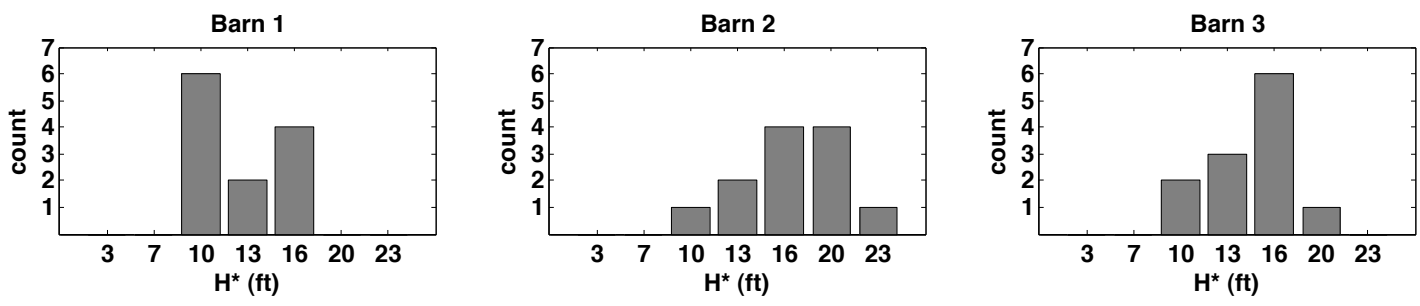

Figure 6.8: Barn height estimate histograms. The data are that of Table 6.1.

some simulations, the registration step produced a misalignment as shown in Figure 6.7b. When this occurred, the mutual information $\mathcal{I}^{\prime}\left(\mathrm{v}_{0}, \mathrm{v}_{\boldsymbol{b}}\right)$ was set to zero.

Three sets of simulations were generated for each candidate value of $H$. This resulted in a total of twelve height estimates for each barn, one from each combination of SAR image and simulation set. The results are shown in Table 6.1 and in histogram form in Figure 6.8. The results were not consistent in the estimate of the height $H$. The histograms show that, on average, the estimated height of Barn 1 was less than the estimated heights of Barns 2 and 3, which agree with the optical photo of the barns. 
Table 6.1: Barn Height Extraction Results

Table values are height rounded to the nearest foot.

\begin{tabular}{c|ccc|ccc|ccc} 
& \multicolumn{3}{|c}{ Barn 1 } & \multicolumn{3}{c}{ Barn 2 } & \multicolumn{3}{c}{ Barn 3 } \\
\hline SAR Image & \multicolumn{3}{|c}{ Simulation Set } & \multicolumn{3}{c}{ Simulation Set } & \multicolumn{3}{c}{ Simulation Set } \\
CPI & 1 & 2 & 3 & 1 & 2 & 3 & 1 & 2 & 3 \\
\hline 1 & 13 & 10 & 16 & 16 & 16 & 20 & 16 & 16 & 20 \\
2 & 10 & 10 & 10 & 13 & 10 & 16 & 13 & 10 & 16 \\
3 & 13 & 10 & 16 & 13 & 20 & 16 & 13 & 16 & 16 \\
4 & 16 & 10 & 16 & 20 & 23 & 20 & 13 & 10 & 16
\end{tabular}
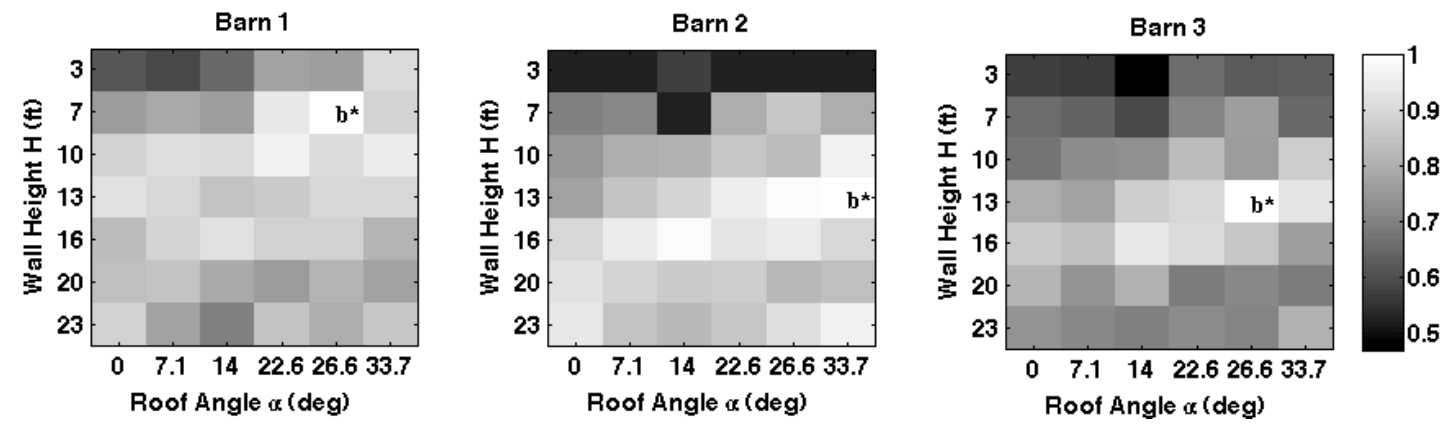

Figure 6.9: The similarity maps for the barns using CPI 3.

For the second experiment, both the height $H$ and roof angle $\alpha$ parameters were varied. The candidate height values were the same as those of the first experiment. The candidate roof angles were those of Table 4.1, excluding the very steep roof angles. One simulation was generated for each combination of height and roof angle. The LiMIT image from CPI 3 was used as the SAR image. The resulting similarity maps are shown in Figure 6.9. The mutual information similarity measure did not vary smoothly with the parameters. The height estimates were lower than the average estimates from the first experiment. The roof angle estimate for Barn 2 does not agree with the optical photo. 


\subsection{Summary}

The mutual information method was applied to two canonical examples of buildings that represent extremes of building size and orientation. In the case of the L-building, the building is large and oriented such that the illuminated area of the building is maximized. In the case of the barns, the buildings are small and their orientation limits the available roof angle information.

The method performed well in the L-building case. The estimates of the building shape $\boldsymbol{b}^{*}$ were consistent for four different LiMIT images, and agreed with available a priori knowledge. This case demonstrated that both the height and the roof angle of a building can be extracted simultaneously, when the other parameters are known. The mutual information similarity measure varied smoothly in the two-dimensional parameter space, suggesting that an optimized search could be used instead of specifying the candidate values at the start.

The method performed poorly in the case of the barns. The height estimates were not consistent for the four different LiMIT images. The inconsistency could be partlially explained by the variation between the LiMIT images (see Figure 6.6). But the estimates for the same LiMIT image using different simulation sets were also inconsistent.

The registration step is critical. When a misalignment occurs, the mutual information measure of (6.16) is meaningless and the resulting similarity map is unreliable. Successful registration depends on the accuracy of the estimate of the joint probability (6.18). The L-building was large in terms of pixels, and therefore more data points were available for the estimate. Even so, the mutual information measure as a function of translational shift $\tau$ contained many local maxima as evident in Figure 
6.4b. The local maxima arise from partial alignments between the distinct image features; when the features are large and well-defined, then the complete alignment of all the features will produce a strong global maximum. The barns, on the other hand, were small and the features ill-defined. The roof scattering feature of a barn was similar to the random background of the simulation images. A solution may be to reduce the size of the simulation images so that the possible translational shifts are restricted to a few pixels in either direction.

Further investigation is needed to determine which factor - size or feature distinctiveness - is more important for reliable building shape estimates using this method. Useful data for this would be SAR images of a large building - not L-shaped - oriented in the scene as the barns were in the LiMIT imagery and SAR images of a small building oriented at 45 degrees to the sensor so that the scattering from two of the walls is visible in the images. 


\section{Chapter 7}

\section{Conclusion}

\subsection{Summary of Contributions}

- Demonstrated a mutual information method for extracting building height and roof angle from SAR imagery. The demonstration showed that both building height and roof angle can be extracted from a single SAR image, extending previous work that demonstrated height estimation only. Although the accuracy of the estimates could not be verified, the estimates were reasonable in light of available a priori information. The demonstration also revealed that the method performs poorly on small, broadside-oriented buildings. A unique aspect of the demonstration was the use of multiple SAR images of the same building and multiple simulations for each candidate building shape. A SAR image is generated by a random process, and so is the simulation image because the scatterers in the scene are assigned a random phase. Therefore, testing the consistency of the results using multiple images and simulations can provide strong evidence for reliable performance, as in the case of the L-building, or can reveal limitations, as in the case of the barns. 


\section{- Developed new equations relating a building's physical parameters to}

its SAR image features. These relationships have not previously been made explicit. The equations for the widths of the roof and wall image features, (4.12) and (4.11), give the theoretical relationships between these features and a building's parameters. Their usefulness for information extraction is limited because it remains difficult to measure the widths of these overlapping features in a SAR image. However, the equations led to the derivation of bounds on the precision of building parameter information contained in the features. The parameter resolution equations (4.16) and (4.18) establish a reference for evaluating the performance of information extraction methods. The equations also show how parameter resolution is affected by the sensor grazing angle and squint angle, and so could be used to tune these angles prior to a data collection mission.

\section{- Developed a new phase-domain method for simulating the SAR raw} signal. The phase-domain simulation method was shown to produce the same raw signal simulation as the time-domain method, the standard for accurate simulation. The phase-domain method requires 0.5 percent of the number of complex multiplies required by the time-domain method for simulating a $100 \mathrm{x}$ 200 meter scene. In practical terms, the phase-domain simulation took about 30 minutes to simulate the L-building scene response and the time-domain method took over 12 hours on the same desktop computer. Simulation is a key part of the information extraction method demonstrated here, and is a useful tool for many kinds of SAR research. The capability to quickly and accurately simulate detailed scenes with SAR parameters matched to a high-resolution sensor facilitates further development of model-based information extraction methods. 
- Identified ground cover as a significant influence on a building's electromagnetic response. It is well-established that the building's orientation relative to the sensor is a significant influence on the building's electromagnetic response. Here, it was shown that for a building surrounded by asphalt, the double-bounce scattering is insensitive to the building orientation and will be the brightest image feature for any building orientation. For a building surrounded by grass, the double-bounce scattering will be dominant only for building orientations from 0 (broadside) to about 10 degrees, and will be insignificant relative to the wall and roof scattering for other orientations. The results were obtained using the GO-PO model, which has been validated with empirical measurements. The implication is that accounting for ground cover could improve the accuracy of SAR image interpretation and simulation.

\subsection{Future Work}

The mutual information method must be applied to more SAR data to fully characterize its accuracy, precision and operational range. The demonstration here identified best-case and worst-case conditions for the method; future work should be directed toward refining the conditions required for reliable performance. Datasets from multiple sensors with different resolutions would facilitate an empirical verification of the theoretical bounds on the precision of building parameters available in the image features.

Information is contained in the dimensions of the manually selected region of the SAR image containing a building. This information was only partially exploited in the case of the barns, where building shapes whose shadows extended outside 
the region were rejected. The dimensions of the selected regions could be used to provide bounds on the candidate parameter values, and could be used to estimate the footprint parameters $L$ and $W$, which here were specified from another data source. The orientation of the building could also be inferred with the aid of a line detector such as the Hough transform. Exploiting this information about the footprint and orientation would be a step towards fully automating the information extraction method. Another advance would be to automate the selection of the region itself.

Information about the material properties of a building could theoretically be extracted from SAR imagery. Franceschetti demontrated how building height could be estimated from image feature intensity using his equation for the double-bounce scattering $[29,30]$. For the demonstration, the permittivity of the walls and the roughness statistics of the surrounding ground were measured at the site. The demonstration validated the relationship between the material properties and the scattering intensity. This suggests that the composition of a building and the type of surrounding ground could be estimated using the mutual information method demonstrated here 


\section{Bibliography}

[1] Lendaris, G. G., "On systemness and the problem solver: tutorial comments," IEEE Systems, Man and Cybernetics, vol. SMC-16, no. 4, pp. 603-610, 1986.

[2] Irvin, R. and McKeown Jr., D. M., "Methods for exploiting the relationship between buildings and their shadows in aerial imagery," IEEE Transactions on Systems, Man and Cybernetics, vol. 19, no. 6, pp. 1564-1575, 1989.

[3] Mayer, H., "Automatic object extraction from aerial imagery - a survey focusing on buildings," Computer Vision and Image Understanding, vol. 74, no. 2, pp. 138-149, 1999.

[4] Peng, J. and Liu, Y., "Model and context-driven building extraction in dense urban aerial images," International Journal of Remote Sensing, vol. 26, no. 7, pp. 1289-1307, 2005.

[5] Bolter, R. and Leberl, F., "Fusion of multiple view interferometric and slant range SAR data for building reconstruction," in SAR Image Analysis, Modeling, and Techniques III, vol. 4173, Barcelona, Spain, 2000, pp. 241-250.

[6] Bolter, R., "Reconstruction of man-made objects from high resolution SAR images," in 2000 IEEE Aerospace Conference, vol. 3, Big Sky, MT, United states, 2000, pp. 287-292.

[7] Tison, C., Tupin, F., and Maitre, H., "Retrieval of building shapes from shadows in high resolution SAR interferometric images," in IEEE IGARSS, vol. 3, Anchorage, AK, United states, 2004, pp. 1788-1791.

[8] Hill, R., Moate, C., and Blacknell, D., "Estimating building dimensions from synthetic aperture radar image sequences," IET Radar, Sonar and Navigation, vol. 2, no. 3, pp. 189-199, 2008.

[9] Jahangir, M., Blacknell, D., Moate, C., and Hill, R., "Extracting information from shadows in SAR imagery," in International Conference on Machine Vision, Islamabad, Pakistan, 2007, pp. 107-112. 
[10] Cellier, F., Oriot, H., and Nicolas, J.-M., "Hypothesis management for building reconstruction from high resolution InSAR imagery," in IEEE IGARSS, Denver, CO, United states, 2006, pp. 3639-3642.

[11] Xu, F. and Jin, Y.-Q., "Automatic reconstruction of building objects from multiaspect meter-resolution SAR images," IEEE Transactions on Geoscience and Remote Sensing, vol. 45, no. 7, pp. 2336-2353, 2007.

[12] Quartulli, M. and Datcu, M., "Stochastic geometrical modeling for built-up area understanding from a single SAR intensity image with meter resolution," IEEE Transactions on Geoscience and Remote Sensing, vol. 42, no. 9, pp. 1996-2003, 2004.

[13] Lacroix, V. and Acheroy, M., "Feature extraction using the constrained gradient," ISPRS Journal of Photogrammetry and Remote Sensing, vol. 53, no. 2, pp. 85-94, 1998.

[14] Michaelsen, E., Soergel, U., and Thoennessen, U., "Perceptual grouping for automatic detection of man-made structures in high-resolution SAR data," in Pattern Recognition in Remote Sensing, vol. 27, 2006, pp. 218-225.

[15] Luttrell, S. P. and Oliver, C. J., "Prior knowledge in synthetic-aperture radar processing," Journal of Physics D: Applied Physics, vol. 19, pp. 333-356, 1986.

[16] Ayyub, B. M. and Klir, G. J., Uncertainty modeling and analysis in engineering and the sciences. CRC Press, 2006.

[17] Franceschetti, G., Iodice, A., Riccio, D., and Ruello, G., "An analytical and numerical framework for interpretation of SAR images of urban areas," in IEEE IGARSS, vol. 3, Toronto, Ont., Canada, 2002, pp. 1926-1928.

[18] Franceschetti, G., Guida, R., Iodice, A., and Riccio, D., "Electromagnetic modelling for information extraction from high resolution SAR images of urban areas," in IEEE IGARSS, vol. 1, Boston, MA, United states, 2008, pp. I78-I81.

[19] Thiele, A. et al., "Feature extraction of gable-roofed buildings from multi-aspect high-resolution InSAR data," in IEEE IGARSS, Barcelona, Spain, 2008, pp. 262-265.

[20] Thiele, A., Cadario, E., Schulz, K., and Soergel, U., "Analysis of gable-roofed building signature in multiaspect InSAR data," IEEE Geoscience and Remote Sensing Letters, vol. 7, no. 1, pp. 83-87, 2010.

[21] Brunner, D., Lemoine, G., and Bruzzone, L., "Building height retrieval from airborne VHR SAR imagery based on an iterative simulation and matching procedure," Remote Sensing for Environmental Monitoring, Proceedings of SPIE, vol. 7110, 2008. 
[22] — - "Estimation of building heights from detected dual-aspect VHR SAR imagery using an iterative simulation and matching procedure in combination with functional analysis," in IEEE Radar Conference, Pasadena, CA, United States, 2009, pp. MTT-S.

[23] Dong, Y., Forster, B., and Ticehurst, C., "Radar backscatter analysis for urban environments," International Journal of Remote Sensing, vol. 18, no. 6, pp. 13511364, 1997.

[24] Xia, Z.-G. and Henderson, F. M., "Understanding the relationships between radar response patterns and the bio- and geophysical parameters of urban areas," IEEE Transactions on Geoscience and Remote Sensing, vol. 35, no. 1, pp. 93101, 1997.

[25] Franceschetti, G., Iodice, A., and Riccio, D., "A canonical problem in electromagnetic backscattering from buildings," IEEE Transactions on Geoscience and Remote Sensing, vol. 40, no. 8, pp. 1787-1801, 2002.

[26] Al-Nuaimi, M. and Ding, M., "Prediction models and measurements of microwave signals scattered from buildings," IEEE Transactions on Antennas and Propagation, vol. 42, no. 8, pp. 1126-1137, 1994.

[27] Brunner, D. et al., "Analysis of the double bounce scattering mechanism of buildings in VHR SAR data," in Image and Signal Processing for Remote Sensing XIV, vol. 7109, Cardiff, Wales, United kingdom, 2008.

[28] Brunner, D., Bruzzone, L., Ferro, A., and Lemoine, G., "Analysis of the reliability of the double bounce scattering mechanism for detecting buildings in VHR SAR images," in IEEE Radar Conference, Pasadena, CA, United States, 2009, pp. MTT-S.

[29] Franceschetti, G. et al., "Accuracy of building height estimation from SAR images," in IEEE IGARSS, Denver, CO, United states, 2006, pp. 3647-3650.

[30] — - "Building feature extraction via a deterministic approach: Application to real high resolution SAR images," in IEEE IGARSS, Barcelona, Spain, 2008, pp. 2681-2684.

[31] Guida, R., Iodice, A., Riccio, D., and Stilla, U., "Model-based interpretation of high-resolution SAR images of buildings," IEEE Journal of Selected Topics in Applied Earth Observations and Remote Sensing, vol. 1, no. 2, pp. 107-119, 2008.

[32] Balz, T., Becker, S., Haala, N., and Kada, M., "Using real-time SAR simulation to assist pattern recognition applications in urban areas," Pattern Recognition and Image Analysis, vol. 18, no. 3, pp. 412-416, 2008. 
[33] Dogan, O. and Kartal, M., "Time domain SAR raw data simulation of distributed targets," Eurasip Journal on Advances in Signal Processing, 2010.

[34] Vandewal, M., Speck, R., and Suss, H., "Efficient and precise processing for squinted spotlight SAR through a modified stolt mapping," Eurasip Journal on Advances in Signal Processing, vol. 2007, p. 59704, 2007.

[35] Vandewal, M., Speck, R., and Suc, H., "Efficient SAR raw data generation including low squint angles and platform instabilities," IEEE Geoscience and Remote Sensing Letters, vol. 5, no. 1, pp. 26-30, 2008.

[36] Mori, A. and Vita, F. D., "A time-domain raw signal simulator for interferometric SAR," IEEE Transactions on Geoscience and Remote Sensing, vol. 42, no. 9, pp. 1811-1817, 2004.

[37] Allan, J. M., Collins, M. J., and Gierull, C., "Computational synthetic aperture radar (cSAR): A flexible signal simulator for multichannel SAR systems," Canadian Journal of Remote Sensing, vol. 36, no. 4, pp. 345-360, 2010.

[38] Liu, H., Zhao, Z., and Jezek, K. C., "Correction of positional errors and geometric distortions in topographic maps and dems using a rigorous SAR simulation technique," Photogrammetric Engineering and Remote Sensing, vol. 70, no. 9, pp. 1031-1042, 2004.

[39] Balz, T. and Stilla, U., "Hybrid GPU-based single- and double-bounce SAR simulation," Ieee Transactions on Geoscience and Remote Sensing, vol. 47, no. 10, pp. 3519-3529, 2009.

[40] Franceschetti, G., Migliaccio, M., Riccio, D., and Schirinzi, G., "SARAS: a synthetic aperture radar (SAR) raw signal simulator," IEEE Transactions on Geoscience and Remote Sensing, vol. 30, no. 1, pp. 110-123, 1992.

[41] Koo, V., Lim, C., and Chan, Y., "ISIM - an integrated SAR product simulator for system designers and researchers," Journal of Electromagnetic Waves and Applications, vol. 21, no. 3, pp. 313-328, 2007.

[42] Franceschetti, G., Migliaccio, M., Riccio, D., and Schirinzi, G., "Three dimensional SAR simulator," in Proceedings of the 20th European Microwave Conference, vol. 1, Budapest, Hungary, 1990, pp. 887-892.

[43] Cimmino, S. et al., "Efficient spotlight SAR raw signal simulation of extended scenes," IEEE Transactions on Geoscience and Remote Sensing, vol. 41, no. 10, pp. 2329-2337, 2003.

[44] Franceschetti, G., Iodice, A., Perna, S., and Riccio, D., "SAR sensor trajectory deviations: Fourier domain formulation and extended scene simulation of raw 
signal," IEEE Transactions on Geoscience and Remote Sensing, vol. 44, no. 9, pp. 2323-2333, 2006.

[45] Wang, Y., Zhang, Z., and Deng, Y., "Squint spotlight SAR raw signal simulation in the frequency domain using optical principles," IEEE Transactions on Geoscience and Remote Sensing, vol. 46, no. 8, pp. 2208-2215, 2008.

[46] Shannon, C. E., "A mathematical theory of communication," Bell System Technical Journal, vol. 27, pp. 379-423, 1948.

[47] Viola, P. A., "Alignment by maximization of mutual information," Ph.D. Dissertation, Massachusetts Institute of Technology, 1995.

[48] Viola, P. and Wells, W., "Alignment by maximization of mutual information," International Journal of Computer Vision, vol. 24, no. 2, pp. 137-154, 1997.

[49] Maes, F. et al., "Multimodality image registration by maximization of mutual information," IEEE Transactions on Medical Imaging, vol. 16, no. 2, pp. 187198, 1997.

[50] Roche, A., Malandain, G., and Ayache, N., "Unifying maximum likelihood approaches in medical image registration," International Journal of Imaging Systems and Technology, vol. 11, pp. 71-80, 2000.

[51] Pluim, J. P., Maintz, J. A., and Viergever, M. A., "Mutual-information-based registration of medical images: A survey," IEEE Transactions on Medical Imaging, vol. 22, no. 8, pp. 986-1004, 2003.

[52] Chen, H.-M., Arora, M., and Varshney, P., "Mutual information-based image registration for remote sensing data," International Journal of Remote Sensing, vol. 24, no. 18, pp. 3701-3706, 2003.

[53] Le Hegarat-Mascle, S. and Seltz, R., "Automatic change detection by evidential fusion of change indices," Remote Sensing of Environment, vol. 91, no. 3-4, pp. 390-404, 2004.

[54] Faur, D., Gavat, I., and Datcu, M., "Mutual information based measure for image content characterization," in 11th Conference of the Spanish Association for Artificial Intelligence, vol. 4177 LNAI, Santiago de Compostela, Spain, 2006, pp. 342-349.

[55] Bitelli, G., Camassi, R., Gusella, L., and Mognol, A., "Damage change detection on urban area: The earthquake case," International Archives of the Photogrammetry, Remote Sensing and Spatial Information Sciences, vol. XXXV, Part B, pp. 692-697, 2004. 
[56] Xie, H., Pierce, L. E., and Ulaby, F. T., "Mutual information based registration of SAR images," in IEEE IGARSS, vol. 6, Toulouse, France, 2003, pp. 40284031.

[57] Shu, L., Tan, T., Tang, M., and Pan, C., "A novel registration method for SAR and SPOT images," in IEEE International Conference on Image Processing, vol. 2, Genova, Italy, 2005, pp. 213-216.

[58] Lixia, S. and Tieniu, T., "SAR and SPOT image registration based on mutual information with contrast measure," in IEEE International Conference on Image Processing, vol. 5, San Antonio, TX, United states, 2006, pp. V429-V432.

[59] Suri, S. and Reinartz, P., "Mutual-information-based registration of TerraSAR$\mathrm{X}$ and Ikonos imagery in urban areas," IEEE Transactions on Geoscience and Remote Sensing, vol. 48, no. 2, pp. 939-949, 2010.

[60] Nies, H. et al., "Image registration of terrasar-x data using different information measures," in IEEE IGARSS, vol. 4, Boston, MA, United states, 2008, pp. IV419-IV422.

[61] Yu, X., Sun, H., and Cao, Y., "SAR image registration combining watershed segmentation and mutual information," in SAR and Multispectral Image Processing, vol. 6043 II, Wuhan, China, 2005.

[62] Yu, X., Yu, W., Guo, L., and Xiang, Y., "A novel segmentation-based mutual information image registration for SAR images," in IET Conference on Wireless, Mobile and Sensor Networks, Shanghai, China, 2007, pp. 829-832.

[63] Brown, L. G., "A survey of image registration techniques," ACM Computing Surveys, vol. 24, no. 4, pp. 325-376, 1992.

[64] Chen, H.-M., Varshney, P. K., and Arora, M. K., "Performance of mutual information similarity measure for registration of multitemporal remote sensing images," IEEE Transactions on Geoscience and Remote Sensing, vol. 41, no. 11, 2003.

[65] Tsang, L., Theory of Microwave Remote Sensing. New York: Wiley, 1985.

[66] Balanis, C. A., Advanced Engineering Electromagnetics. Wiley, 1989.

[67] Ishamaru, A., Wave propagation and scattering in random media. New York: Academic Press, 1978, vol. 2.

[68] Verly, J., "Physical optics polarization scattering matrix for a right-angle dihedral," MIT Lincoln Laboratory, Tech. Rep., February 1995. 
[69] Blejer, D. J., "Physical optics polarization scattering matrix of a square trihedral corner reflector," MIT Lincoln Laboratory, Tech. Rep., June 1987.

[70] Cover, T. M. and Thomas, J. A., Elements of Information Theory. New York: John Wiley \& Sons, 1991.

[71] Jakowatz, C. V. et al., Spotlight-Mode Synthetic Aperture RADAR: A Signal Processing Approach. Norwell, MA: Kluwer Academic Publishers, 1996.

[72] Duhamel, P. and Vetterli, M., "Fast Fourier transforms: a tutorial review and a state of the art," Signal Processing, vol. 19, pp. 259-299, 1990.

[73] Lugosi, G. and Nobel, A., "Consistency of data-driven histogram methods for density estimation and classification," The Annals of Statistics, vol. 24, no. 2, pp. 687-706, 1996.

[74] Sturges, H. A., "The choice of a class interval," Journal of American Statistical Association, pp. 65-66, 1926.

[75] Scott, D. W., "Optimal and data-based histograms," Biometrika, vol. 66, no. 3, pp. 605-610, 1979.

[76] — , "Sturges' rule," Wiley Interdisciplinary Reviews: Computational Statistics, vol. 1, no. 3, pp. 303-306, 2009.

[77] Legg, P. A., Rosin, P. L., Marshall, D., and Morgan, J. E., "Improving accuracy and efficiency of registration by mutual information using Sturges' histogram rule," in Medical Image Understanding and Analysis, Aberystwyth, Wales, United Kingdom, 2007, pp. 26-30. 